\title{
Characterization and representation of the lower semicontinuous envelope of the elastica functional
}

\section{Caractérisation et représentation de l'enveloppe semi-continue inférieure de la fonctionnelle de l'elastica}

\author{
G. Bellettini ${ }^{a}$, L. Mugnai ${ }^{b}$ \\ a Dipartimento di Matematica, Università di Roma "Tor Vergata", via della Ricerca Scientifica, 00133 Roma, Italy \\ ${ }^{\mathrm{b}}$ Dipartimento di Matematica, Università di Pisa, via Buonarroti 2, 56127 Pisa, Italy \\ Received 22 May 2003; received in revised form 18 December 2003; accepted 30 January 2004
}

Available online 9 June 2004

\begin{abstract}
We characterize the lower semicontinuous envelope $\overline{\mathcal{F}}$ of the functional $\mathcal{F}(E):=\int_{\partial E}\left[1+\left|\kappa_{\partial E}\right|^{p}\right] d \mathcal{H}^{1}$, defined on boundaries of sets $E \subset \mathbb{R}^{2}$, where $\kappa_{\partial E}$ denotes the curvature of $\partial E$ and $p>1$. Through a desingularization procedure, we find the domain of $\overline{\mathcal{F}}$ and its expression, by means of different representation formulas.

(C) 2004 L'Association Publications de l'Institut Henri Poincaré. Published by Elsevier B.V. All rights reserved
\end{abstract}

\section{Résumé}

On caractérise l'enveloppe semi-continue inférieure $\overline{\mathcal{F}}$ de la fonctionnelle $\mathcal{F}(E):=\int_{\partial E}\left[1+\left|\kappa_{\partial E}\right|^{p}\right] d \mathcal{H}^{1}$, définie sur la classe des frontières des domaines $E \subset \mathbb{R}^{2}$, où $\kappa_{\partial E}$ dénote la courbure de $\partial E$ et $p>1$. Grâce à une méthode de désingularisation, on trouve le domaine de $\overline{\mathcal{F}}$ et son expression, à l'aide de différentes formules de représentation.

(C) 2004 L'Association Publications de l'Institut Henri Poincaré. Published by Elsevier B.V. All rights reserved

MSC: 49J45; 49Q20

Keywords: Semicontinuity; Curvature depending functionals; Elastica; Relaxation

\section{Introduction}

In recent years a growing attention has been devoted to integral energies depending on curvatures of a manifold; besides the geometric interest of functionals such as the Willmore functional [2,24,25], curvature depending energies arise in models of elastic rods [11,15,17], and in image segmentation [8,18-23]. In the case of plane

E-mail addresses: belletti@mat.uniroma2.it (G. Bellettini), mugnai@mail.dm.unipi.it (L. Mugnai). 
curves the main example is the functional of the so-called elastic curves $[11,13]$ which reads as $\int\left[1+|\ddot{\gamma}|^{2}\right] d s$. This functional is the starting point of the research pursued in this paper. Let us consider the functional

$$
\mathcal{F}(E):=\int_{\partial E}\left[1+\left|\kappa_{\partial E}(z)\right|^{p}\right] d \mathcal{H}^{1}(z),
$$

where $E \subset \mathbb{R}^{2}$ is a bounded open subset of class $\mathcal{C}^{2}, p>1$ is a real number, $\kappa_{\partial E}(z)$ is the curvature of $\partial E$ at $z$ and $\mathcal{H}^{1}$ is the one-dimensional Hausdorff measure in $\mathbb{R}^{2}$.

The map $\mathcal{F}$, considered as a function of the set $E$ rather than of its boundary $\partial E$, appears in problems of computer vision $[8,22,23]$ and of image inpainting [3,18,19]. It is a simplified version of the building block appearing in the model suggested in [23] to segment an image taking into account the relative depth of the objects.

One of the motivations of looking at $\mathcal{F}$ as a function of the sets $E$, which are endowed with the $L^{1}$-topology, comes from the above mentioned applications, where one is typically interested in minimizing $\mathcal{F}$ coupled with a bulk term; for instance, one looks for solutions of problems of the form

$$
\inf _{E \in \mathcal{M}}\left\{\overline{\mathcal{F}}(E)+\int_{E} g(z) d z\right\},
$$

for an appropriate given bulk energy $g$, where $\overline{\mathcal{F}}$ stands for the $L^{1}$-lower semicontinuous envelope of $\mathcal{F}$, defined on the class $\mathcal{M}$ of all measurable subsets of $\mathbb{R}^{2}$. Another motivation for adopting this point of view is represented by a conjecture in [14], where the approximation of the Willmore functional through elliptic second order functionals is addressed.

The choice of the $L^{1}$ topology quickly yields the existence of minimizers of (2) under rather mild assumptions on $g$, see the discussion in [4]; however, it is clear that, being the $L^{1}$ topology of sets a very weak topology (especially for functionals depending on second derivatives), several difficulties arise when trying to characterize the domain of $\overline{\mathcal{F}}$ and to find its value.

The study of the properties of $\overline{\mathcal{F}}$ was initiated by Bellettini, Dal Maso and Paolini in [4]. After proving that $\mathcal{F}=\overline{\mathcal{F}}$ on regular sets [4, Theorem 3.2], the authors exhibited several examples of nonsmooth sets $E$ having $\overline{\mathcal{F}}(E)<+\infty$, see for instance Fig. 1. However, some of these examples are rather pathological (for instance, sets $E$ that locally around a point $p$ have a qualitative shape as in Fig. 2) and show that the characterization of the domain of $\overline{\mathcal{F}}$ is not an easy task.

Let us briefly recall the partial characterization of $\overline{\mathcal{F}}$ obtained in [4, Theorems 4.1, 6.2]. If $E \subset \mathbb{R}^{2}$ is such that $\overline{\mathcal{F}}(E)<+\infty$, then there exists a system of curves $\Gamma=\left\{\gamma_{1}, \ldots, \gamma_{m}\right\}$ (that is, a finite family of constant speed immersions of the unit circle $S^{1}$, see Definition 2.2) such that $\gamma_{i} \in H^{2, p}\left(S^{1}\right)$, the union of the supports $\bigcup_{i=1}^{m}\left(\gamma_{i}\right)=$ : $(\Gamma)$ covers $\partial E$ and has no transversal crossings, and $E$ coincides in $L^{1}\left(\mathbb{R}^{2}\right)$ with $\left\{z \in \mathbb{R}^{2} \backslash(\Gamma): \mathcal{I}(\Gamma, z)=1\right\}=$ :

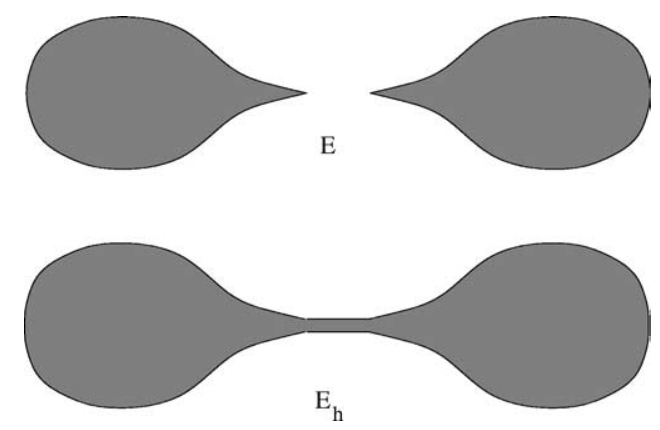

Fig. 1. The set $E$ is made by two connected components having one cusp point. The sequence $\left\{E_{h}\right\}$ consists of smooth sets converging to $E$ in $L^{1}\left(\mathbb{R}^{2}\right)$ whose energy $\mathcal{F}$ is uniformly bounded with respect to $h$. Hence $\overline{\mathcal{F}}(E)<+\infty$. 
$A_{\Gamma}$, where $\mathcal{I}(\Gamma, \cdot)$ is the index of $\Gamma$ (see Definition 2.7). As a partial converse of the previous result, given a system of curves $\Gamma=\left\{\gamma_{1}, \ldots, \gamma_{m}\right\} \in H^{2, p}\left(S_{1}^{1} \times \cdots \times S_{m}^{1}\right)$, if $\Gamma$ has no transversal crossings and self-intersects tangentially only at a finite number of points, then $\overline{\mathcal{F}}\left(A_{\Gamma}^{o}\right)<+\infty$, where $A_{\Gamma}^{o}:=\left\{z \in \mathbb{R}^{2}: \mathcal{I}(\Gamma, z) \equiv 1(\bmod 2)\right\}$. We stress that the hypothesis of finiteness for the set of self-intersection points of $\Gamma$ (which in the sequel will be called the singular set of $\Gamma$ and denoted by $\left.\operatorname{Sing}_{\Gamma}\right)$ is an effective restriction since it may happen that $\mathcal{H}^{1}\left(\operatorname{Sing}_{\Gamma}\right)>0$, as was shown in [4, Example 1, p. 271]. To conclude the list of the known results concerning the domain of $\overline{\mathcal{F}}$, in [4, Theorem 6.4] it is proved that, if $\partial E$ can be locally represented as the graph of a function of class $H^{2, p}$ up to a finite number of "simple cusp points" (see Definition 2.33) then $\overline{\mathcal{F}}(E)<+\infty$ is equivalent to the condition that the total number of cusps is even. Finally, as far as the value of $\overline{\mathcal{F}}$ is concerned, in [4, Theorem 7.3] it is proved that $\overline{\mathcal{F}}(\cdot, \Omega)$ does not admit an integral representation, where $\overline{\mathcal{F}}(\cdot, \Omega)$ is the localization of $\overline{\mathcal{F}}$ on an open set $\Omega$. This phenomenon is due to the presence, in the computation of $\overline{\mathcal{F}}(E, \Omega)$, of hidden curves (not in general contained in $\partial E$ ) which are a reminiscence of the limit of the boundaries $\partial E_{h}$ of a minimizing sequence $\left\{E_{h}\right\}$. Such hidden curves could be put in relation with the problem of reconstructing the contours of an object which is partially occluded by another object closer to the observer [6].

Eventually, the computation of $\overline{\mathcal{F}}(E)$ is carried on in [4, Theorem 7.2] in one case only, i.e., when $\partial E$ has only two cusps which are positioned in a very special way (as in Fig. 1), the proof being not adaptable to more general configurations.

The aim of this paper is to answer the above discussed questions left open in the paper [4]. More precisely, we can

- characterize the domain of $\overline{\mathcal{F}}$, thus removing the crucial finiteness assumption in Theorem 6.2 of [4], through a desingularization procedure on systems of curves $\Gamma$ having an infinite number of singularities;

- exhibit different representation formulas for $\overline{\mathcal{F}}$ (obviously not integral representation in the usual sense), making computable (at least in principle) the value of $\overline{\mathcal{F}}(E)$ for nonsmooth sets $E$;

- describe the structure of the boundaries of the sets $E$ with $\overline{\mathcal{F}}(E)<+\infty$, and extend [4, Theorem 6.4] to boundaries with more general singular points rather than simple cusp points.

We remark that, in the discussion of the above items, we also characterize the structure of those systems of curves which are obtained as weak $H^{2, p}$ limits of boundaries of smooth bounded open sets.

Let us briefly describe the content of the paper. In Sections 2, 3 we prove some preliminary results, leading to a characterization of the singular set of systems of curves. To explain with some details our results in the subsequent sections, let us introduce some definitions. If $\Gamma=\left\{\gamma_{1}, \ldots, \gamma_{m}\right\}$ is a system of curves of class $H^{2, p}$, we let $\mathcal{F}(\Gamma):=\sum_{i=1}^{m} \int\left[1+\left|\ddot{\gamma}_{i}\right|^{p}\right] d s$. We say that two systems $\Gamma, \widetilde{\Gamma}$ of curves are equivalent (and we write $\Gamma \sim \widetilde{\Gamma}$ ) if their traces coincide, i.e., $(\Gamma)=(\widetilde{\Gamma})$ and if $\sharp\left\{\Gamma^{-1}(p)\right\}=\sharp\left\{\widetilde{\Gamma}^{-1}(p)\right\}$ for any $p \in(\Gamma)$. It is not difficult to show that if $\Gamma \sim \widetilde{\Gamma}$, then $\mathcal{F}(\Gamma)=\mathcal{F}(\widetilde{\Gamma})$. In Theorem 5.1 and Corollary 5.2 we show that, given an arbitrary system of curves $\Gamma=\left\{\gamma_{1}, \ldots, \gamma_{m}\right\}$ of class $H^{2, p}$, without transversal crossings, there exists a system of curves $\widetilde{\Gamma} \sim \Gamma$ which is the strong $H^{2, p}$-limit of a sequence $\left\{\partial E_{N}\right\}$ of boundaries of smooth, open, bounded sets such that

$$
\lim _{N \rightarrow \infty} \mathcal{F}\left(E_{N}\right)=\mathcal{F}(\Gamma), \quad \lim _{N \rightarrow \infty} E_{N}=A_{\Gamma}^{o} \quad \text { in } L^{1}\left(\mathbb{R}^{2}\right) .
$$

This approximation result generalizes [4, Theorem 6.2], since no finiteness assumptions on $\operatorname{Sing}_{\Gamma}$ is required. The proof, which is quite involved, requires a desingularization of $\Gamma$ around the accumulation points of $\operatorname{Sing}_{\Gamma}$, and is based on several preliminary lemmata, see Section 4. Observe that in Theorem 5.1 we show that among all systems

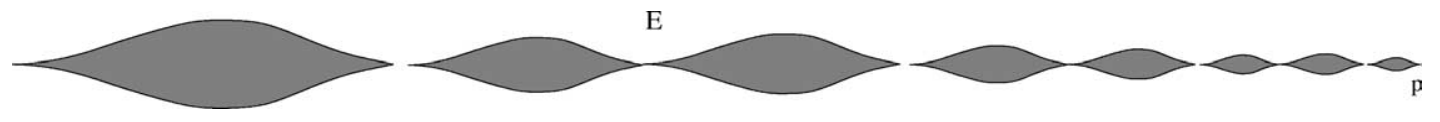

Fig. 2. The grey region denotes (possibly a part of) the set $E$. If $E$, locally around the singular point $p$ (which is an accumulation point of singular points of $\partial E$ ), behaves as in the figure, it may happen that $\overline{\mathcal{F}}(E)<+\infty$. 
of curves of class $H^{2, p}$ without transversal crossings, those which have finite singular set are a dense subset in the energy norm. Hence we also have that every $E$ with $\overline{\mathcal{F}}(E)<+\infty$ can be approximated both in $L^{1}\left(\mathbb{R}^{2}\right)$ and in energy by a sequence of subsets $\left\{E_{N}\right\}$ such that $\operatorname{Sing}_{\partial E_{N}}$ consists only of a finite number of cusps and branch points (see again Definition 2.33).

In Section 6 we give some representation formulas for $\overline{\mathcal{F}}$. In particular, in Proposition 6.1 we show that

$$
\overline{\mathcal{F}}(E)=\min \{\mathcal{F}(\Gamma): \Gamma \in \mathcal{A}(E)\},
$$

where $\Gamma \in \mathcal{A}(E)$ if and only if $(\Gamma) \supseteq \partial E$ and $E=A_{\Gamma}$ in $L^{1}\left(\mathbb{R}^{2}\right)$. This formula is much in the spirit of [10, Corollary 5.4], where a similar, but in some sense weaker, result is proved in the framework of Geometric Measure Theory. Motivated by the density result of subsets with finite singular set given in Theorem 5.1 and Corollary 5.2, in Theorem 6.3 we prove that if $E$ has a finite number of singular points then the collection $\mathcal{Q}_{\text {fin }}(E)$ of all systems $\Gamma \in \mathcal{A}(E)$ with finite singular set is dense in $\mathcal{A}(E)$ with respect to the $H^{2, p}$-weak convergence and in energy. Moreover

$$
\overline{\mathcal{F}}(E)=\inf \left\{\mathcal{F}(\Gamma): \Gamma \in \mathcal{Q}_{\text {fin }}(E)\right\} .
$$

Theorem 6.3 is stronger than Theorem 5.1, since the approximating sequence now must fulfill the additional constraint of being made of elements of $\mathcal{Q}_{\text {fin }}(E)$. Moreover Theorem 6.3 turns out to be the key technical tool for proving the results of Section 8. Note carefully that the minimum in (4) in general is not attained, as we show in Proposition 8.8.

In Section 7 the regularity of minimizers $\Gamma$ for problem (3) is studied in the case $p=2$. The main result of this section is Theorem 7.1 where we show that any solution $\Gamma$ of the minimum problem (3) has, out of $\partial E$, a finite singular set and consists of pieces of elastic curves.

In Section 8 we focus our attention on subsets $E$ with finite singular set and with $\overline{\mathcal{F}}(E)<+\infty$. The main result of this section is Theorem 8.6, where we give a (close to optimal) representation formula for $\overline{\mathcal{F}}(E)$. Precisely, we prove that

$$
\overline{\mathcal{F}}(E)=\int_{\operatorname{Reg}_{\partial E}}\left[1+\left|\kappa_{\partial E}(z)\right|^{p}\right] d \mathcal{H}^{1}(z)+2 \min _{\sigma \in \Sigma(E)} \mathcal{F}(\sigma) .
$$

Here $\operatorname{Reg}_{\partial E}$ denotes the regular part of the boundary of $E, \Sigma(E)$ is (roughly speaking) the class of all curves $\sigma$ of class $H^{2, p}$ connecting the singular points of $\partial E$ in an appropriate way, which do not cross transversally each other and do not cross transversally $\partial E$. This result is a wide generalization of the example discussed in [4], where the set $E$ had only two cusps and a very specific geometry.

\section{Notation and preliminaries}

A plane curve $\gamma:[0, a] \rightarrow \mathbb{R}^{2}$ of class $\mathcal{C}^{1}$ is said to be regular if $\frac{d \gamma(t)}{d t} \neq 0$ for every $t \in[0,1]$. Each closed regular curve $\gamma:[0,1] \rightarrow \mathbb{R}^{2}$ will be identified, in the usual way, with a map $\gamma: S^{1} \rightarrow \mathbb{R}^{2}$, where $S^{1}$ denotes the oriented unit circle. By $(\gamma)=\gamma([0,1])=\{\gamma(t): t \in[0,1]\}$ we denote the trace of $\gamma$ and by $l(\gamma)$ its length; $s$ denotes the arc length parameter and $\dot{\gamma}, \ddot{\gamma}$ the first and second derivative of $\gamma$ with respect to $s$. Let us fix a real number $p>1$ and let $p^{\prime}$ be such that $1 / p+1 / p^{\prime}=1$. If the second derivative $\ddot{\gamma}$ in the sense of distributions belongs to $L^{p}$, then the curvature $\kappa(\gamma)$ of $\gamma$ is given by $|\ddot{\gamma}|$, and

$$
\|\kappa(\gamma)\|_{L^{p}}^{p}=\int_{] 0, l(\gamma)[}|\ddot{\gamma}|^{p} d s<+\infty
$$

in this case we say that $\gamma$ is a curve of class $H^{2, p}$, and we write $\gamma \in H^{2, p}$. Moreover, we put

$$
\mathcal{F}(\gamma):=l(\gamma)+\|\kappa(\gamma)\|_{L^{p}}^{p}
$$


If $z \in \mathbb{R}^{2} \backslash(\Gamma), \mathcal{I}(\gamma, z)$ is the index of $\gamma$ with respect to $z$ [7].

For any $C \subset \mathbb{R}^{2}$ we denote by $\operatorname{int}(C)$ the interior of $C$, by $\bar{C}$ the closure of $C$, and by $\partial C$ the topological boundary of $C$. All sets we will consider are assumed to be measurable.

For every set $E \subset \mathbb{R}^{2}$ let $\chi_{E}$ denote its characteristic function, that is $\chi_{E}(z)=1$ if $z \in E, \chi_{E}(z)=0$ if $z \notin E$; for any $z_{0} \in \mathbb{R}^{2}, \rho>0, B_{\rho}\left(z_{0}\right):=\left\{z \in \mathbb{R}^{2}:\left|z-z_{0}\right|<\rho\right\}$ is the ball centered at $z_{0}$ with radius $\rho$.

Definition 2.1. We say that $E \subset \mathbb{R}^{2}$ is of class $H^{2, p}$ (respectively $\mathcal{C}^{k}, k \geqslant 1$ ) if $E$ is open and if, for every $z \in \partial E$, the set $E$ can be locally represented as the subgraph of a function of class $H^{2, p}$ (respectively $\mathcal{C}^{k}$ ) with respect to a suitable coordinate system.

Let $E \subset \mathbb{R}^{2}$ be a set of class $H^{2, p}$. Since $\partial E$ can be locally viewed as the graph of an $H^{2, p}$ function, we can define, locally, the curvature $\kappa_{\partial E}$ of $\partial E$ at $\mathcal{H}^{1}$-almost every point of $\partial E$ using the classical formulas involving the second derivatives. One can readily check that the definition of $\kappa_{\partial E}$ does not depend on the choice of the coordinate system used to represent $\partial E$ as a graph, and also that $\kappa_{\partial E} \in L^{p}\left(\partial E, \mathcal{H}^{1}\right)$.

Given a set $E \subset \mathbb{R}^{2}$, we define

$$
E^{*}:=\left\{z \in \mathbb{R}^{2}: \exists r>0:\left|B_{r}(z) \backslash E\right|=0\right\},
$$

$|\cdot|$ denoting the Lebesgue measure. If $\Delta$ stands for the symmetric difference between sets and $|E \Delta F|=0$, then $E^{*}=F^{*}$.

Let $\mathcal{M}$ be the collection of all measurable subsets of $\mathbb{R}^{2}$. We can identify $\mathcal{M}$ with a closed subset of $L^{1}\left(\mathbb{R}^{2}\right)$ through the map $E \mapsto \chi_{E}$. The $L^{1}\left(\mathbb{R}^{2}\right)$ topology induced by this map on $\mathcal{M}$ is the same topology induced on $\mathcal{M}$ by the metric $\left(E_{1}, E_{2}\right) \rightarrow\left|E_{1} \Delta E_{2}\right|$, where $E_{1}, E_{2} \in \mathcal{M}$.

Now we define the map $\mathcal{F}: \mathcal{M} \rightarrow[0,+\infty]$ as follows:

$$
\mathcal{F}(E):= \begin{cases}\int_{\partial E}\left[1+\left|\kappa_{\partial E}(z)\right|^{p}\right] d \mathcal{H}^{1}(z) & \text { if } E \text { is a bounded open set of class } \mathcal{C}^{2}, \\ +\infty & \text { elsewhere on } \mathcal{M} .\end{cases}
$$

We call $L^{1}$-relaxed functional of $\mathcal{F}$, and denote it by $\overline{\mathcal{F}}$, the lower semicontinuous envelope of $\mathcal{F}$ with respect to the topology of $L^{1}\left(\mathbb{R}^{2}\right)$. It is known that, for every $E \in \mathcal{M}$, we have

$$
\overline{\mathcal{F}}(E)=\inf \left\{\liminf _{h \rightarrow \infty} \mathcal{F}\left(E_{h}\right): E_{h} \rightarrow E \operatorname{in} L^{1}\left(\mathbb{R}^{2}\right) \text { as } h \rightarrow \infty\right\} .
$$

\subsection{Systems of curves}

In this subsection we list all definitions and known facts on systems of curves used throughout the paper, and we prove some preliminary results.

Definition 2.2. By a system of curves we mean a finite family $\Gamma=\left\{\gamma_{1}, \ldots, \gamma_{m}\right\}$ of closed regular curves of class $\mathcal{C}^{1}$ such that $\left|\frac{d \gamma_{i}}{d t}\right|$ is constant on $[0,1]$ for any $i=1, \ldots, m$. Denoting by $\mathbb{S}$ the disjoint union of $m$ circles $S_{1}^{1}, \ldots, S_{m}^{1}$ of unitary length, we shall identify $\Gamma$ with the map $\Gamma: \mathbb{S} \mapsto \mathbb{R}^{2}$ defined by $\Gamma_{\mid S_{i}^{1}}:=\gamma_{i}$ for $i=1, \ldots, m$. The trace $(\Gamma)$ of $\Gamma$ is defined as $(\Gamma):=\bigcup_{i=1}^{m}\left(\gamma_{i}\right)$.

By a system of curves of class $H^{2, p}(\mathbb{S})$ we mean a system $\Gamma=\left\{\gamma_{1}, \ldots, \gamma_{m}\right\}$ such that each $\gamma_{i}$ is of class $H^{2, p}\left(S_{i}^{1}\right)$. In this case we shall write $\Gamma \in H^{2, p}(\mathbb{S})$.

Definition 2.3. By a disjoint system of curves we mean a system of curves $\Gamma=\left\{\gamma_{1}, \ldots, \gamma_{m}\right\}$ such that $\left(\gamma_{i}\right) \cap\left(\gamma_{j}\right)=$ $\emptyset$ for any $i, j=1, \ldots, m, i \neq j$. 
Definition 2.4. We say that a system of curves $\Gamma=\left\{\gamma_{1}, \ldots, \gamma_{m}\right\}$ is without crossings if $\frac{d \gamma_{i}\left(t_{1}\right)}{d t}$ and $\frac{d \gamma_{j}\left(t_{2}\right)}{d t}$ are parallel, whenever $\gamma_{i}\left(t_{1}\right)=\gamma_{j}\left(t_{2}\right)$ for some $i, j \in\{1, \ldots, m\}$ and $t_{1}, t_{2} \in[0,1]$.

Definition 2.5. If $\Gamma=\left\{\gamma_{1}, \ldots, \gamma_{m}\right\}$ is a system of curves of class $H^{2, p}$, we define

$$
l(\Gamma):=\sum_{i=1}^{m} l\left(\gamma_{i}\right), \quad\|\kappa(\Gamma)\|_{L^{p}}^{p}:=\sum_{i=1}^{m} \int_{] 0, l\left(\gamma_{i}\right)[}\left|\ddot{\gamma}_{i}(s)\right|^{p} d s,
$$

and

$$
\mathcal{F}(\Gamma)=\sum_{i=1}^{m} \mathcal{F}\left(\gamma_{i}\right):=\sum_{i=1}^{m} l\left(\gamma_{i}\right)+\left\|\kappa\left(\gamma_{i}\right)\right\|_{L^{p}}^{p} .
$$

As $\left|\frac{d \gamma_{i}}{d t}\right|$ is constant on $[0,1]$, we have $s(t)=t l\left(\gamma_{i}\right)$, hence

$$
\int_{0, l\left(\gamma_{i}\right)[}\left|\ddot{\gamma}_{i}\right|^{p} d s=l\left(\gamma_{i}\right)^{1-2 p} \int_{] 0,1[}\left|\frac{d^{2} \gamma_{i}}{d t^{2}}\right|^{p} d t .
$$

Given a set $A=A_{1} \times \cdots \times A_{m} \subseteq \mathbb{S}$ and a system of curves $\Gamma=\left\{\gamma_{1}, \ldots, \gamma_{m}\right\} \in H^{2, p}(\mathbb{S})$, we fix the following notation:

$$
\mathcal{F}(\Gamma, A):=\sum_{i=1}^{m} \int_{A_{i}}\left\{\left|\frac{d \gamma_{i}}{d t}\right| d t+l\left(\gamma_{i}\right)^{1-2 p} \int_{A_{i}}\left|\frac{d^{2} \gamma_{i}}{d t^{2}}\right|^{p}\right\} d t .
$$

Remark 2.6. With a small abuse of notation, with the same letter $\mathcal{F}$ we denote a functional defined on $\mathcal{M}$ and a functional defined on regular $H^{2, p}$ curves.

Definition 2.7. Let $\Gamma=\left\{\gamma_{1}, \ldots, \gamma_{m}\right\}$ be a system of curves. If $z \in \mathbb{R}^{2} \backslash(\Gamma)$ we define the index of $z$ with respect to $\Gamma$ as $\mathcal{I}(\Gamma, z):=\sum_{i=1}^{m} \mathcal{I}\left(\gamma_{i}, z\right)$.

Definition 2.8. Let $E \subset \mathbb{R}^{2}$ be a bounded open set of class $\mathcal{C}^{1}$. We say that a disjoint system of curves $\Gamma$ is an oriented parametrization of $\partial E$ if each curve of the system is simple, $(\Gamma)=\partial E$, and, in addition,

$$
E=\left\{z \in \mathbb{R}^{2} \backslash \partial E: \mathcal{I}(\Gamma, z)=1\right\}, \quad \mathbb{R}^{2} \backslash \bar{E}=\left\{z \in \mathbb{R}^{2} \backslash \partial E: \mathcal{I}(\Gamma, z)=0\right\} .
$$

In [4, Proposition 3.1] it is proved that any bounded subset $E$ of $\mathbb{R}^{2}$ of class $H^{2, p}$ (respectively $\mathcal{C}^{2}$ ) admits an oriented parametrization of class $H^{2, p}$ (respectively $\mathcal{C}^{2}$ ).

Definition 2.9. We say that a sequence $\left\{\Gamma_{h}\right\}$ of systems of curves of class $H^{2, p}$ converges weakly (respectively strongly) in $H^{2, p}$ to a system of curves $\Gamma=\left\{\gamma_{1}, \ldots, \gamma_{m}\right\}$ of class $H^{2, p}$ if the number of curves of each system $\Gamma_{h}$ equals the number of curves of $\Gamma$ for $h$ large enough, i.e., $\Gamma_{h}=\left\{\gamma_{1}^{h}, \ldots, \gamma_{m}^{h}\right\}$, and, in addition, $\gamma_{i}^{h}$ converges weakly (respectively strongly) to $\gamma_{i}$ in $H^{2, p}$ as $h \rightarrow \infty$ for any $i=1, \ldots, m$.

If $\left\{\Gamma_{h}\right\}$ weakly converges to $\Gamma=\left\{\gamma_{1}, \ldots, \gamma_{m}\right\}$ in $H^{2, p}$, then

$$
\gamma_{i}^{h} \rightarrow \gamma_{i} \quad \text { in } \mathcal{C}^{1} \text { as } h \rightarrow \infty,
$$

for any $i=1, \ldots, m$. In particular, $l\left(\gamma_{i}^{h}\right) \rightarrow l\left(\gamma_{i}\right)$ as $h \rightarrow \infty$. 
The following result is proved in [4, Theorem 3.1]; it states the coercivity of the functional $\mathcal{F}$ with respect to the weak $H^{2, p}$ convergence of systems of curves.

Theorem 2.10. Let $\left\{\Gamma_{h}\right\}$ be a sequence of systems of curves of class $H^{2, p}$ such that all $\left(\Gamma_{h}\right)$ are contained in a bounded subset of $\mathbb{R}^{2}$ independent of $h$ and

$$
\sup _{h \in \mathbb{N}} \mathcal{F}\left(\Gamma_{h}\right)<+\infty .
$$

Then $\left\{\Gamma_{h}\right\}$ has a subsequence which converges weakly in $H^{2, p}$ to a system of curves $\Gamma$.

Definition 2.11. We say that $\Gamma$ is a limit system of curves of class $H^{2, p}$ if $\Gamma$ is the weak limit of a sequence $\left\{\Gamma_{h}\right\}$ of oriented parametrizations of bounded open sets of class $H^{2, p}$.

Definition 2.12. We say that a system of curves $\Gamma$ verifies the finiteness property in an open set $U \subset \mathbb{R}^{2}$ if there exists a finite set $S \subset U$ such that $(\Gamma) \backslash S$ is a one-dimensional embedded submanifold of $\mathbb{R}^{2}$ of class $\mathcal{C}^{1}$.

\subsection{Nice rectangles}

Definition 2.13. Let $\Gamma=\left\{\gamma_{1}, \ldots, \gamma_{m}\right\}$ be a system of curves of class $H^{2, p}$ without crossings. Let $p \in(\Gamma)$, let $\tau(p)$ be a unit tangent vector to $(\Gamma)$ at $p$, and let $\tau^{\perp}(p)$ be the rotation of $\tau(p)$ of $\pi / 2$ around the origin in counterclockwise order. We say that $R(p)$ is a nice rectangle for $(\Gamma)$ at $p$ if

$$
R(p)=\left\{z \in \mathbb{R}^{2}: z=p+l \tau(p)+d \tau^{\perp}(p),|l| \leqslant a,|d| \leqslant b\right\},
$$

where $a>0$ and $b>0$ are selected in such a way that $(\Gamma) \cap R(p)$ is given by the union of the cartesian graphs, with respect to the tangent line $T_{p}(\Gamma)$ to $(\Gamma)$ at $p$, of a finite number of functions $\left\{f_{1}, \ldots, f_{r}\right\}$ such that $\operatorname{graph}\left(f_{l}\right)$ does not intersect the two sides of $R(p)$ which are parallel to $T_{p}(\Gamma)$ for every $l=\{1, \ldots, r\}$.

Remark 2.14. By regularity properties of systems of curves of class $H^{2, p}$ without crossings, one readily checks that each point $p \in(\Gamma)$ admits a nice rectangle $R(p)$ at $p$. Moreover, if $\Lambda \in H^{2, p}$ and $(\Lambda) \subseteq(\Gamma)$, then $R(p)$ is a nice rectangle at $p$ also for $\Lambda$.

Let $p \in(\Gamma)$; when we write a nice rectangle $R(p)$ at $p$ for $(\Gamma)$ in the form $R(p)=[-a, a] \times[-b, b]$, we implicitly assume that $p$ is the origin of the coordinates, that $T_{p}(\Gamma)$ is the $x$-axis, and that $\tau^{\perp}(p)$ agrees with the vector $(0,1)$. In this case we also set $R^{+}(p):=[0, a] \times[-b, b]$ and $R^{-}(p):=[-a, 0] \times[-b, b]$.

\subsection{Density function of a system of curves}

Definition 2.15. Let $\Gamma$ be a system of curves of class $H^{2, p}$. We define the density function $\theta_{\Gamma}$ of $\Gamma$ as

$$
\theta_{\Gamma}:(\Gamma) \rightarrow \mathbb{N} \cup\{+\infty\}, \quad \theta_{\Gamma}(z):=\sharp\left\{\Gamma^{-1}(z)\right\},
$$

$\sharp$ denoting the counting measure.

Lemma 2.16. Let $\Gamma=\left\{\gamma_{1}, \ldots, \gamma_{m}\right\}$ be a system of curves of class $H^{2, p}$. Then there exists $M \in \mathbb{N}$ depending only on $m$ and on $\mathcal{F}(\Gamma)$ such that

$$
\sharp\left\{\gamma_{i}^{-1}(p)\right\} \leqslant M \quad \forall p \in\left(\gamma_{i}\right), \forall i=1, \ldots, m .
$$

Proof. The statement is a consequence of step 1 in the proof of Theorem 9.1 in [6].

Remark 2.17. As a direct consequence of Lemma 2.16 we obtain that if $\Gamma$ is a system of curves of class $H^{2, p}$ then $\theta_{\Gamma}(z)$ is uniformly bounded with respect to $z \in(\Gamma)$. 
2.4. Definitions of $A_{\Gamma}, A_{\Gamma}^{o}, \mathcal{A}(E), \mathcal{Q}_{\mathrm{fin}}(E), \mathcal{A}^{o}(E)$

If $\Gamma$ is a system of curves of class $H^{2, p}$, in the following we set

$$
\begin{aligned}
& A_{\Gamma}:=\left\{z \in \mathbb{R}^{2} \backslash(\Gamma): \mathcal{I}(\Gamma, z)=1\right\}, \\
& A_{\Gamma}^{o}:=\left\{z \in \mathbb{R}^{2} \backslash(\Gamma): \mathcal{I}(\Gamma, z) \equiv 1(\bmod 2)\right\} .
\end{aligned}
$$

Remark 2.18. If $\Gamma$ is a limit system of curves of class $H^{2, p}$ then $\mathcal{I}(\Gamma, z) \in\{0,1\}$ for any $z \in \mathbb{R}^{2} \backslash(\Gamma)$, see [4]; in particular $A_{\Gamma}=A_{\Gamma}^{o}$.

Definition 2.19. Let $E \subset \mathbb{R}^{2}$. We denote by $\mathcal{A}(E)$ the collection of all limit systems of curves $\Gamma$ of class $H^{2, p}$ satisfying

$$
(\Gamma) \supseteq \partial E^{*}, \quad E^{*}=\operatorname{int}\left(A_{\Gamma} \cup(\Gamma)\right) .
$$

We indicate by $\mathcal{Q}_{\text {fin }}(E)$ the collection of all systems $\Gamma \in \mathcal{A}(E)$ verifying the finiteness property in $\mathbb{R}^{2} .{ }^{1}$ We denote by $\mathcal{A}^{o}(E)$ the collection of all systems of curves $\Gamma$ of class $H^{2, p}$ satisfying

$$
(\Gamma) \supseteq \partial E^{*}, \quad E^{*}=\operatorname{int}\left(A_{\Gamma}^{o} \cup(\Gamma)\right) .
$$

Note that the elements of $\mathcal{A}^{o}(E)$ are not, in general, limit systems of curves. Moreover $\mathcal{Q}_{\text {fin }}(E) \subseteq \mathcal{A}(E) \subseteq$ $\mathcal{A}^{o}(E)$. Finally, in view of Theorem 2.22 and (7) (respectively (8)), for every $\Gamma \in \mathcal{A}(E)$ (respectively $\Gamma \in \mathcal{A}^{o}(E)$ ), it holds $\left|A_{\Gamma} \Delta E\right|=0$ (respectively $\left|A_{\Gamma}^{o} \Delta E\right|=0$ ), provided $\overline{\mathcal{F}}(E)<+\infty$.

Remark 2.20. If $\Gamma \in \mathcal{A}(E)$ (respectively $\Gamma \in \mathcal{A}^{o}(E)$ ) and $F \subset \mathbb{R}^{2}$ is such that $|E \Delta F|=0$, then $\Gamma \in \mathcal{A}(F)$ (respectively $\Gamma \in \mathcal{A}^{o}(F)$ ).

\subsection{On sets $E$ with $\overline{\mathcal{F}}(E)<+\infty$}

Definition 2.21. Let $C$ be a subset of $\mathbb{R}^{2}$. We say that $C$ has a continuous unoriented tangent if at each point $z \in C$ the tangent cone $T_{C}(z)$ to $C$ at $z$ (see [4, Definition 4.1]) is a straight line and the map $T_{C}: z \mapsto T_{C}(z)$ from $C$ into the real projective space $\mathbb{P}^{1}$ is continuous.

The following results are proved in [4, Theorems 4.1, 6.2, 7.3].

Theorem 2.22. Let $E \subset \mathbb{R}^{2}$ be such that $\overline{\mathcal{F}}(E)<+\infty$. Then $E^{*}$ is bounded, open, $\left|E \Delta E^{*}\right|=0, \mathcal{H}^{1}\left(\partial E^{*}\right)<+\infty$ and $\partial E^{*}$ has a continuous unoriented tangent. Moreover

$$
\overline{\mathcal{F}}(E) \geqslant \inf \{\mathcal{F}(\Gamma): \Gamma \in \mathcal{A}(E)\},
$$

hence in particular $\mathcal{A}(E)$ is nonempty.

Remark 2.23. Let $\Gamma$ be a system of curves of class $H^{2, p}$ without crossings and define

$$
E:=A_{\Gamma}^{o}, \quad F:=\left\{z \in \mathbb{R}^{2} \backslash(\Gamma): \mathcal{I}(\Gamma, z) \equiv 0(\bmod 2)\right\} .
$$

Then, as noticed in [4], $E, E^{*}, F, F^{*}$ are open, $E^{*}$ is bounded, $\left|E \Delta E^{*}\right|=0$ and

$$
\partial E^{*}=\partial F^{*}=\left\{z \in \mathbb{R}^{2}: 0<\left|B_{r}(z) \cap E\right|<\left|B_{r}(z)\right| \forall r>0\right\}=\partial E \cap \partial F \subseteq(\Gamma), \quad E^{*}=\operatorname{int}\left(A_{\Gamma}^{o} \cup(\Gamma)\right) .
$$

Therefore $\Gamma \in \mathcal{A}^{o}(E)$.

\footnotetext{
${ }^{1}$ Let us remark that in [4] the set $\mathcal{Q}_{\text {fin }}(E)$ was denoted by $\mathcal{Q}(E)$.
} 
Theorem 2.24. Let $\Gamma$ be a system of curves of class $H^{2, p}$ without crossings and satisfying the finiteness property. Then

$$
\overline{\mathcal{F}}\left(A_{\Gamma}^{o}\right)<+\infty,
$$

hence there exists a sequence $\left\{E_{h}\right\}$ of bounded open sets of class $\mathcal{C}^{2}$ converging to $A_{\Gamma}^{o}$ in $L^{1}\left(\mathbb{R}^{2}\right)$ and such that $\sup _{h \in \mathbb{N}} \mathcal{F}\left(E_{h}\right)<\infty$. In addition, there exist oriented parametrizations $\Gamma_{h}$ of $\partial E_{h}$ defined on the same parameter space $\mathbb{S}$, such that $\left\{\Gamma_{h}\right\}$ converges strongly in $H^{2, p}(\widetilde{\mathbb{S}})$ to a system of curves equivalent to $\Gamma$ (see Definition 2.30), defined on $\widetilde{\mathbb{S}}$, and whose trace contains $\partial A_{\Gamma}^{o}$.

Theorem 2.25. There exists a set $E \subset \mathbb{R}^{2}$ such that $\overline{\mathcal{F}}(E)<+\infty$ and $\overline{\mathcal{F}}(E, \cdot)$ is not subadditive.

\subsection{Regular and singular points of $\Gamma$. Equivalent systems}

Definition 2.26. Let $\Gamma$ be a system of curves of class $H^{2, p}$ without crossings and let $p \in(\Gamma)$. We say that $p$ is a regular point for $(\Gamma)$ if there exists a neighborhood $U_{p}$ of $p$ such that $(\Gamma) \cap U_{p}$ is the graph of a function of class $H^{2, p}$ with respect to $T_{p}(\Gamma)$. We say that $p \in(\Gamma)$ is a singular point of $(\Gamma)$ if $p$ is not a regular point of $(\Gamma)$. We indicate by $\operatorname{Reg}_{\Gamma}$ the set of all regular points of $(\Gamma)$ and by $\operatorname{Sing}_{\Gamma}=(\Gamma) \backslash \operatorname{Reg}_{\Gamma}$ the set of all singular points of $(\Gamma)$.

Remark 2.27. If $\Gamma \in H^{2, p}(\mathbb{S})$ is a system of curves, then $\operatorname{Reg}_{\Gamma} \neq \emptyset$. This is obvious if $\operatorname{Sing}_{\Gamma}=\emptyset$. If $\operatorname{Sing}_{\Gamma} \neq \emptyset$, let $p \in \operatorname{Sing}_{\Gamma}$ and let $R(p)=[-a, a] \times[-b, b]$ be a nice rectangle for $(\Gamma)$ at $p$, and write

$$
R(p) \cap(\Gamma)=\bigcup_{l=1}^{r} \operatorname{graph}\left(f_{l}\right), \quad f_{l} \in H^{2, p}(]-a, a[) .
$$

We proceed by induction over the number $r$ of graphs. Suppose $r=2$. As $p \in \operatorname{Sing}_{\Gamma}$, there exists $\left.\xi_{1} \in\right]-a, a$ [ such that $f_{1}\left(\xi_{1}\right) \neq f_{2}\left(\xi_{1}\right)$; hence we can find an open neighborhood $\left.U \subset\right]-a, a$ [ of $\xi_{1}$ such that $f_{1}(x) \neq f_{2}(x)$ for every $x \in U$. Therefore $\operatorname{Reg}_{\Gamma} \cap R(p) \supset\left\{\left(x, f_{l}(x)\right): x \in U\right\} \neq \emptyset, l=1,2$. Assume that when $R(p) \cap(\Gamma)$ consists of $r>2$ graphs of $H^{2, p}$ functions, then $\operatorname{Reg}_{\Gamma} \cap R(p) \neq \emptyset$. Suppose that $(\Gamma) \cap R(p)$ consists of $r+1$ graphs of $H^{2, p}$ functions $f_{1}, \ldots, f_{r+1}$. Define

$$
J:=\{x \in]-a, a\left[: f_{1}(x) \notin\left\{f_{2}(x), \ldots, f_{r+1}(x)\right\}\right\} .
$$

If $J=\emptyset$ then graph $\left(f_{1}\right)$ is contained in the union of the remaining $r$ graphs and the thesis follows by the induction hypothesis. Otherwise there is $\xi_{1} \in J$ and an open neighborhood $\left.U \subset\right]-a, a$ [ of $\xi_{1}$ such that $f_{1}(x) \notin\left\{f_{2}(x), \ldots, f_{r+1}(x)\right\}$ for every $x \in U$. Hence $\operatorname{Reg}_{\Gamma} \cap R(p) \supset\left\{\left(x, f_{1}(x)\right): x \in U\right\} \neq \emptyset$.

By an arc of regular points we mean a connected component of $(\Gamma)$ consisting of regular points of $(\Gamma)$. If $p \in(\Gamma)$ by $B_{\rho}^{+}(p)$ (respectively $\left.B_{\rho}^{-}(p)\right)$ we mean $\left\{z \in B_{\rho}(p):(z-p) \cdot \tau(p) \geqslant 0\right\}$ (respectively $\{z \in$ $\left.\left.B_{\rho}(z):(z-p) \cdot \tau(p) \leqslant 0\right\}\right)$, where $\tau(p)$ is a unit vector parallel to $d \Gamma / d t$ in $p$.

Definition 2.28. We say that $p \in \operatorname{Sing}_{\Gamma}$ is a node of $(\Gamma)$ if there exists $N_{p} \in \mathbb{N}, N_{p}>1$, such that for any $\rho>0$ sufficiently small either $B_{\rho}^{+}(p) \cap(\Gamma) \backslash\{p\}$ or $B_{\rho}^{-}(p) \cap(\Gamma) \backslash\{p\}$ consists of the union of $N_{p}$ arcs of regular points for $(\Gamma)$ which do not intersect each other. We indicate by $\operatorname{Nod}_{\Gamma}$ the set of the nodes of $(\Gamma)$.

Fig. 3 explains the meaning of the definition of node.

Remark 2.29. Since in the definition of regular point (respectively singular point and node) only the set $(\Gamma)$ is involved and not the map $\Gamma$, similar definitions can be given for every (immersed) one-dimensional submanifold of $\mathbb{R}^{2}$ of class $\mathcal{C}^{1}$ without crossings. 


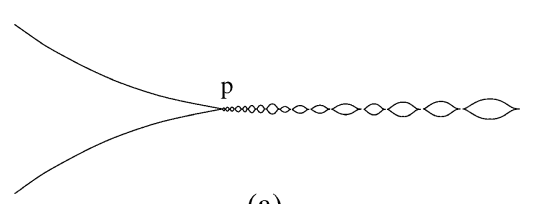

(a)

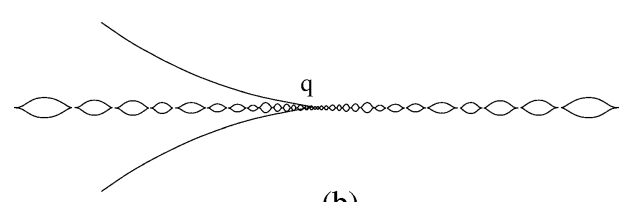

(b)

Fig. 3. The point $p$ in (a) is a node of $(\Gamma)$, while the point $q$ in (b) is not a node of $(\Gamma)$.

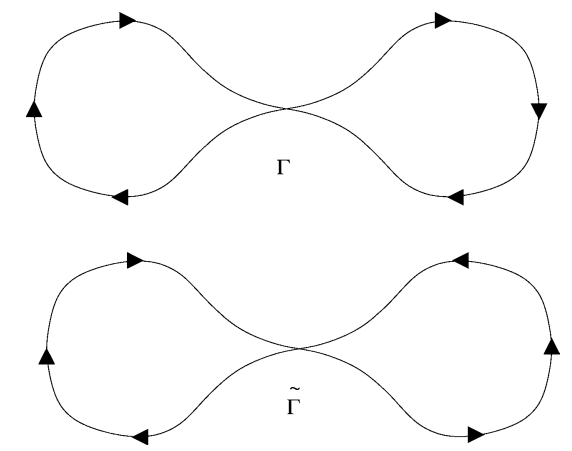

Fig. 4. Two equivalent systems of curves $\Gamma$ and $\widetilde{\Gamma}$; observe that $\Gamma$ is a limit system of curves, while $\widetilde{\Gamma}$ is not a limit system of curves. In particular $\widetilde{\Gamma} \in \mathcal{A}^{o}(E) \backslash \mathcal{A}(E)$ (the set $E$ is the interior of the two drops).

Definition 2.30. Let $\Gamma \in H^{2, p}(\mathbb{S})$ and $\widetilde{\Gamma} \in H^{2, p}(\widetilde{\mathbb{S}})$ be two systems of curves without crossings. We say that $\Gamma$ is equivalent to $\widetilde{\Gamma}$, and we write $\Gamma \sim \widetilde{\Gamma}$, if $(\Gamma)=(\widetilde{\Gamma})$ and $\theta_{\Gamma}=\theta_{\widetilde{\Gamma}}$ on $(\Gamma)$.

If $\Gamma \in \mathcal{A}(E)$ and if $\widetilde{\Gamma} \sim \Gamma$, then $\widetilde{\Gamma}$ does not necessarily belong to $\mathcal{A}(E)$, since in general $\widetilde{\Gamma}$ is not a limit system of curves. In Fig. 4 we show two equivalent systems of curves $\Gamma$ and $\widetilde{\Gamma}$, with $\Gamma \in \mathcal{A}(E)$, such that $\widetilde{\Gamma}$ is not a limit system of curves. Eventually, observe that if $\Gamma \sim \widetilde{\Gamma}$ then $A_{\Gamma}^{o}=A_{\widetilde{\Gamma}}^{o}$.

\subsection{Singular points of $\partial E^{*}$. Cusps and branch points}

Definition 2.31. Let $E$ be an open subset of $\mathbb{R}^{2}$ such that $\partial E$ has continuous unoriented tangent, and let $p \in \partial E$. We say that $p$ is a regular point of $\partial E$ if there exists a neighborhood $U_{p}$ of $p$ such that $U_{p} \cap E$ is the subgraph of a function locally defined over $T_{p}(\partial E)$. We will indicate by $\operatorname{Reg}_{\partial E}$ the set of all regular points of $\partial E$. We say that $p \in \partial E$ is a singular point of $\partial E$ (and write $p \in \operatorname{Sing}_{\partial E}$ ) if $p \notin \operatorname{Reg}_{\partial E}$.

Remark 2.32. If $E \subset \mathbb{R}^{2}$ is such that $\overline{\mathcal{F}}(E)<+\infty$, by Theorem 2.22, near every regular point $p$, the boundary of the set $E^{*}$ can be represented as the graph of an $H^{2, p}$ function with respect to $T_{p}(\partial E)$, see also Lemma 4.3 below.

Definition 2.33. Let $E$ be an open subset of $\mathbb{R}^{2}$ with continuous unoriented tangent, and let $p \in \partial E$. Suppose that there are $\rho>0$ and an integer $k \geqslant 2$ such that either $B_{\rho}^{+}(p) \cap \partial E=\bigcup_{l=1}^{k} \operatorname{graph}\left(f_{l}\right)$ or $B_{\rho}^{-}(p) \cap \partial E=$ $\bigcup_{l=1}^{k} \operatorname{graph}\left(f_{l}\right)$, where the $f_{l}$ are functions defined on $T_{p}(\partial E)$ whose graphs meet each other only at $p$. If $k$ is 


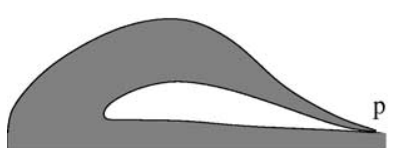

(a)

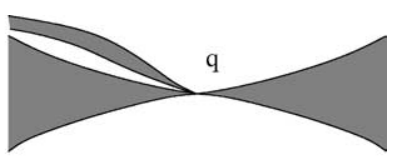

(b)

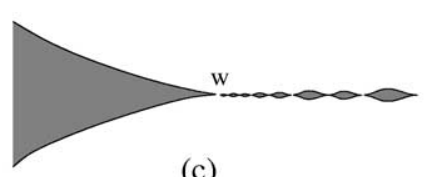

(c)

Fig. 5. The grey region locally stands for the set $E$. The point $p$ of (a) is a branch point, but not a cusp point ( $k=3$ ). The point $q$ of (b) is a cusp point but not a simple cusp point, and the same happens for the point $w$ in (c).

even we say that $p$ is a cusp. If $k$ is odd we say that $p$ is a branch point. If $k=2$ and either $B_{\rho}^{+}(p) \cap \partial E=\{p\}$ or $B_{\rho}^{-}(p) \cap \partial E=\{p\}$, then we say that $p$ is a simple cusp point.

The definition of the set $\operatorname{Nod}_{\partial E}$ of the nodes of $\partial E$ is the same as Definition 2.28 where we replace $\Gamma$ by $\partial E$.

Each connected component of the set $E$ in Fig. 1 has a simple cusp; in Fig. 5 we show examples of branch points, and of cusp points which are not simple cusp points.

Remark 2.34. Let $E$ be such that $\overline{\mathcal{F}}(E)<+\infty, p \in \operatorname{Nod}_{\partial E^{*}}, \Gamma \in \mathcal{A}^{o}(E)$ and let $R$ be a nice rectangle for $(\Gamma)$ at $p$. As noticed in [4, p. 269], the function $\theta_{\Gamma}$ is odd on the regular points of $\partial E^{*}$. Since $\Gamma$ verifies the train tracks property in $R$ (see Definition 3.6 below) we can conclude that

- the point $p$ is always a cusp or a branch point, and cannot be a cusp and a branch point simultaneously, since the constants $k$ corresponding to $B_{\rho}^{+}(p) \cap \partial E$ and to $B_{\rho}^{-}(p) \cap \partial E$ have the same parity;

- if $p$ is a cusp (respectively a branch) point then $\theta_{\Gamma}(p)$ is even (respectively odd). Conversely if $p \in \operatorname{Nod}_{\partial E^{*}}$ and $\theta_{\Gamma}(p)$ is even (respectively odd) then $p$ is a cusp (respectively a branch) point.

\section{Some useful results on systems of curves}

Proposition 3.1. Let $\Gamma$ be a system of curves of class $H^{2, p}$ without crossings. Then $\operatorname{Nod}_{\Gamma}$ is at most countable. Moreover $\operatorname{Sing}_{\Gamma}$ has empty interior,

$$
\operatorname{Sing}_{\Gamma}=\overline{\operatorname{Nod}_{\Gamma}}
$$

and

$$
\overline{\operatorname{Reg}_{\Gamma}}=(\Gamma) .
$$

Remark 3.2. It may happen that $\mathcal{H}^{1}\left(\operatorname{Sing}_{\Gamma}\right)>0$ (see [4, Example 1, p. 271]), therefore in this case $\operatorname{Sing}_{\Gamma} \neq \operatorname{Nod}_{\Gamma}$ and $\operatorname{Sing}_{\Gamma}$ is not countable.

Proof. It is obvious that every node of $(\Gamma)$, or any accumulation point of nodes of $(\Gamma)$, is a singular point, so that $\overline{\operatorname{Nod}_{\Gamma}} \subseteq \operatorname{Sing}_{\Gamma}$.

Let us prove the opposite inclusion. Let $p \in \operatorname{Sing}_{\Gamma}$. We can select a nice rectangle $R=[-a, a] \times[-b, b]$ centered at $p=0$ where $(\Gamma)$ consists of a finite union of $r>1$ graphs of $H^{2, p}$ functions defined on $R \cap T_{p}(\Gamma)$ 
and all passing through the point $p$. In particular, in $[0, a-\varepsilon] \times[-b, b]$ the set $(\Gamma)$ cannot be represented as the graph of one function only for any $\varepsilon \in[0, a[$.

We now reason by induction on the number of graphs. Suppose first that $[0, a] \times[-b, b] \cap(\Gamma)=\operatorname{graph}\left(f_{1}\right) \cup$ $\operatorname{graph}\left(f_{2}\right)$, and set $\alpha_{1}(x):=\left(x, f_{1}(x)\right), \alpha_{2}(x):=\left(x, f_{2}(x)\right), x \in[0, a]$. Define

$$
I:=\left\{x \in[0, a]: f_{1}(x) \neq f_{2}(x)\right\} .
$$

As $f_{1}$ and $f_{2}$ are continuous, $I$ is open, therefore it is the union of a possibly countable number of connected components $I_{h}$. It is clear that, if $x$ belongs to the boundary of one of the $I_{h}$, then $\left(x, f_{1}(x)\right)$ is a node. Since $f_{1}(0)=f_{2}(0)$, it follows that $\alpha_{1}^{-1}(p)$ cannot belong to the interior of any of the $I_{h}$. Hence there are only two possibilities: either $\alpha_{1}^{-1}(p)$ belongs to the boundary of one of the $I_{h}$, and then $p \in \operatorname{Nod}_{\Gamma}$, or $\alpha_{1}^{-1}(p)$ is an accumulation point of boundary points of the intervals $I_{h}$, and so $p \in \overline{\operatorname{Nod}}_{\Gamma}$.

Assume that when $R \cap(\Gamma)$ consists of $r>2$ graphs of functions of class $H^{2, p}$ then $p \in \operatorname{Sing}_{\Gamma} \operatorname{implies}$ $p \in \overline{\operatorname{Nod}}_{\Gamma}$. Suppose that $R \cap(\Gamma)$ consists of $r+1$ graphs of functions $f_{1}, \ldots, f_{r+1}$ of class $H^{2, p}$. Define

$$
J:=\left\{x \in[0, a]: f_{1}(x) \notin\left\{f_{2}(x), \ldots, f_{r+1}(x)\right\}\right\} .
$$

Then $J$ is open. If $J$ is empty, then the graph of $f_{1}$ is contained in the union of the remaining $r$ graphs, and the thesis follows by the induction hypothesis. Therefore we can suppose that $J$ is nonempty. Define $\sigma_{2}:=\sup J>0$, and consider the connected component $] \sigma_{1}, \sigma_{2}\left[\right.$ of $J$ having $\sigma_{2}$ as the right boundary point. Note that $0 \leqslant \sigma_{1}<\sigma_{2}$. We divide the proof into two cases.

Case 1. $\sigma_{1}=0$. If $p$ is a regular point for $\bigcup_{j=2}^{r+1} \operatorname{graph}\left(f_{j}\right)$, then it is a node for $\bigcup_{j=1}^{r+1} \operatorname{graph}\left(f_{j}\right)$, and hence $p \in \operatorname{Nod}_{\Gamma}$. If $p$ is a singular point for $\bigcup_{j=2}^{r+1} \operatorname{graph}\left(f_{j}\right)$, then $p$ is a node (or an accumulation point of nodes) of $\bigcup_{j=2}^{r+1} \operatorname{graph}\left(f_{j}\right)$ by the induction assumption. Therefore $p$ is a node (or an accumulation point of nodes) of $\bigcup_{j=1}^{r+1} \operatorname{graph}\left(f_{j}\right)$, and hence $p \in \overline{\operatorname{Nod}}_{\Gamma}$.

Case 2. $\sigma_{1}>0$. There are two subcases: either all functions $f_{l}$, with $l \in\{2, \ldots, r+1\}$, whose graph passes through the point $\left(\sigma_{1}, f_{1}\left(\sigma_{1}\right)\right)$ coincide in an interval of the form $] \sigma_{1}, \sigma_{1}+\delta[$, or in any interval of this form there are at least two functions $f_{h}, f_{k}$, with $h, k \in\{2, \ldots, r+1\}$ that do not agree. In the first subcase we have $\left(\sigma_{1}, f_{1}\left(\sigma_{1}\right)\right) \in \operatorname{Nod}_{\Gamma}$. In the second subcase we can select a nice rectangle $R_{1} \subset R$ centered at $\left(\sigma_{1}, f_{1}\left(\sigma_{1}\right)\right)$. Inside $R_{1}$ we can repeat the arguments of case 1 for the functions $\left\{f_{1}, \ldots, f_{r+1}\right\}$. We conclude that $\left(\sigma_{1}, f_{1}\left(\sigma_{1}\right)\right) \in \overline{\operatorname{Nod}_{\Gamma}}$.

Now, using the $\mathcal{C}^{1}$-regularity of the $f_{k}$ and the fact that $f_{k}(p)=f_{j}(p)$ and $f_{k}^{\prime}(p)=f_{j}^{\prime}(p)$ for every $1 \leqslant$ $k, j \leqslant r+1$, we take a countable family of shrinking nice rectangles of the form $\left[-a_{h}, a_{h}\right] \times\left[-b_{h}, b_{h}\right]$, with $a_{h} \downarrow 0$ and $b_{h} \downarrow 0$ as $h \rightarrow \infty$, and repeat the above arguments. In this way we obtain a sequence of points $p_{h}:=\left(\sigma_{1}^{h}, f_{1}\left(\sigma_{1}^{h}\right)\right) \in\left[-a_{h}, a_{h}\right] \times\left[-b_{h}, b_{h}\right] \cap \overline{\operatorname{Nod}}_{\Gamma}$, which converges to $p$ and $p \in \overline{\operatorname{Nod}}_{\Gamma}$. This concludes the proof in case 2, and the proof of (12).

Let us now prove (13). Let $p \in(\Gamma)$; we have to prove that in each neighborhood of $p$ there are regular points of $(\Gamma)$. This is immediate if $p \in \operatorname{Reg}_{\Gamma}$. If $p \in \operatorname{Sing}_{\Gamma}$, then by (12) either $p \in \operatorname{Nod}_{\Gamma}$ or $p$ is an accumulation point of $\operatorname{Nod}_{\Gamma}$; in both cases, from the definition of node, we have that in each neighborhood of $p$ there are regular points of $(\Gamma)$.

To conclude the proof of the proposition, it remains to show that $\operatorname{Nod}_{\Gamma}$ is at most countable. Let $p \in(\Gamma)$ and let $R$ be a nice rectangle centered at $p$. Suppose that $(\Gamma) \cap R=\bigcup_{i=1}^{h} \operatorname{graph}\left(f_{i}\right)$, where $f_{i} \in H^{2, p}\left(T_{p}(\Gamma) \cap R\right)$. If $q \in \operatorname{int}(R)$ is a node of $(\Gamma)$, then there are $k, l \in\{1, \ldots, h\}, k \neq l$, and $\xi_{1} \in[-a, a]$ such that $\left(\xi_{1}, f_{k}\left(\xi_{1}\right)\right)=q$ and

$$
f_{k}\left(\xi_{1}\right)=f_{l}\left(\xi_{1}\right) \quad f_{k}\left(\xi_{1}+x\right) \neq f_{l}\left(\xi_{1}+x\right),
$$

for every $x \in]-\delta, 0[$ or $x \in] 0, \delta\left[\right.$ (where $\delta>0$ is a number small enough). Therefore the point $\xi_{1}$ is a boundary point of some connected component of the set $\left\{x \in[-a, a]: f_{k}(x) \neq f_{l}(x)\right\}$, but this is an open set, and so points of this kind can be at most countable. Now, since we can cover the whole of $(\Gamma)$ with a finite number of nice rectangles, we have that $\operatorname{Nod}_{\Gamma}$ is at most countable. 
Remark 3.3. Since $\operatorname{Sing}_{\Gamma}=\overline{\operatorname{Nod}}_{\Gamma}$ by Proposition 3.1, it is clear that if $(\Gamma)$ verifies the finiteness property in $U$ the set $\operatorname{Sing}_{\Gamma} \cap U$ consists of a finite number of nodes.

As a consequence of Proposition 3.1 we obtain the following

Corollary 3.4. Let $\Gamma \in H^{2, p}(\mathbb{S})$ be a system of curves without crossings and let $p \in \operatorname{Sing}_{\Gamma}$. Then there exists a nice rectangle $R$ for $(\Gamma)$ at $p$ such that

$$
(\Gamma) \cap \partial R \subset \operatorname{Reg}_{\Gamma} .
$$

Proof. Suppose $p=0 \in \operatorname{Sing}_{\Gamma}$ and let $[-a, a] \times[-b, b]$ be a nice rectangle for $(\Gamma)$ at $p$. Let $f_{1}, \ldots, f_{r}$ be a collection of $H^{2, p}(]-a, a[)$ functions such that

$$
\bigcup_{l=1}^{r} \operatorname{graph}\left(f_{l}\right)=([-a, a] \times[-b, b]) \cap(\Gamma) .
$$

Clearly for every $\alpha \in]-a, a[$, the set $[-\alpha, \alpha] \times[-b, b]$ is still a nice rectangle for $(\Gamma)$ at $p$ and $(\Gamma) \cap([-\alpha, \alpha] \times$ $[-b, b])$ is still represented by the graphs of $f_{1}, \ldots, f_{r}$. By (13) we can find $q_{1} \in([-a, a] \times[-b, b]) \cap \operatorname{Reg}_{\Gamma}$. Without loss of generality we can suppose that $q_{1}$ has coordinates $\left(a_{1}, f_{1}\left(a_{1}\right)\right)$ and $\left.a_{1} \in\right] 0, a\left[\right.$. As $\operatorname{Reg}_{\Gamma}$ is an open subset of $(\Gamma)$, we can select an interval $\left.I_{1} \subseteq\right] 0, a$ [ centered at $a_{1}$ such that $\left(x, f_{1}(x)\right) \in \operatorname{Reg}_{\Gamma}$ for every $x \in I_{1}$. From Proposition 3.1 we know that $\operatorname{Sing}_{\Gamma}$ has empty interior in $(\Gamma)$. Therefore we can find $a_{2} \in I_{1}$ such that $\left(a_{2}, f_{2}\left(a_{2}\right)\right) \in \operatorname{Reg}_{\Gamma}$ and then select an interval $I_{2} \subseteq I_{1}$ such that $\left(x, f_{1}(x)\right)$ and $\left(x, f_{2}(x)\right)$ are regular points for every $x \in I_{2}$. Repeating the same argument $r$ times we find a point $\left.\left.a_{r} \in\right] 0, a\right]$ such that $\left(\left\{a_{r}\right\} \times \mathbb{R}\right) \cap(\Gamma)=\left\{z_{1}, \ldots, z_{h}\right\} \subset \operatorname{Reg}_{\Gamma}$. Setting $R:=\left[-a_{r}, a_{r}\right] \times[-b, b]$, we get (14).

Lemma 3.5. Let $\Gamma$ be a system of curves of class $H^{2, p}$. Let $p=0 \in(\Gamma)$ and let $R=[-a, a] \times[-b, b]$ be a nice rectangle for $(\Gamma)$ at $p$. Then

$$
\theta_{\Gamma}(p)=\sum_{z \in(\Gamma) \cap(\{x\} \times[-b, b])} \theta_{\Gamma}(z) \quad \forall x \in[-a, a] .
$$

Proof. Write $\Gamma=\left\{\gamma_{1}, \ldots, \gamma_{m}\right\}$. Let $i \in\{1, \ldots, m\}$ be such that $R \cap \gamma_{i}\left(S_{i}^{1}\right) \neq \emptyset$, and write

$$
\gamma_{i}^{-1}(\operatorname{int}(R))=\bigcup_{l=1}^{M_{i}} I_{i l},
$$

where $M_{i} \leqslant\left\|\theta_{\Gamma}\right\|_{\infty}$ and $I_{i l}$ are the connected components of $\gamma_{i}^{-1}(\operatorname{int}(R))$. Using the fact that $\gamma_{i}$ is a constant speed parametrization we have

$$
\begin{aligned}
& \overline{I_{i l}} \cap \overline{I_{i k}}=\emptyset \quad \forall l \neq k, \\
& \gamma_{i}\left(I_{i l}\right) \subset \operatorname{int}(R) \cap(\Gamma), \quad \gamma_{i}\left(\partial I_{i l}\right) \subset(\Gamma) \cap \partial R \quad \forall l, \\
& \left.I_{i l}=\left(s_{1}, s_{2}\right), \quad \gamma_{i}\left(s_{1}\right) \in\{ \pm a\} \times\right]-b, b\left[\Rightarrow \quad \gamma_{i}\left(s_{2}\right) \in \mp\{a\} \times\right]-b, b[,
\end{aligned}
$$

and $\gamma_{i}$ is injective over each $I_{i l}$, so that we can take $M_{i}=\sharp\left\{\gamma_{i}^{-1}(p)\right\}$. Taking the union over all $i \in\{1, \ldots, m\}$ such that $\left(\gamma_{i}\right) \cap R \neq \emptyset$, we get

$$
\Gamma^{-1}(\operatorname{int}(R))=\bigcup_{i=1}^{m} \bigcup_{l=1}^{\sharp\left\{\gamma_{i}^{-1}(p)\right\}} I_{i l},
$$

and $\theta_{\Gamma}(p)=\sum_{i=1}^{m} \sharp\left\{\gamma_{i}^{-1}(p)\right\}$. Therefore (15) holds. 
Definition 3.6. In the following, we will refer to property (15) as to the train tracks property of $\Gamma$ in $R$.

Proposition 3.7. Let $\Gamma=\left\{\gamma_{1}, \ldots, \gamma_{m}\right\}$ be a system of curves of class $H^{2, p}$. Then

(a) for any $p \in(\Gamma)$ and for any $s_{0} \in S_{j}^{1}$ such that $\gamma_{j}\left(s_{0}\right)=p$ there holds

$$
\theta_{\Gamma}\left(\gamma_{j}\left(s_{0}\right)\right) \geqslant \limsup _{S_{j}^{1} \ni s \rightarrow s_{0}} \theta_{\Gamma}\left(\gamma_{j}(s)\right) .
$$

(b) $\theta_{\Gamma}$ is constant on each connected component of $\operatorname{Reg}_{\Gamma}$.

Proof. (b) Let $p \in \operatorname{Reg}_{\Gamma}$ and let $U_{p}$ be a neighborhood of $p$ such that $(\Gamma) \cap U_{p}$ is the graph of a function of class $H^{2, p}$. Let us select a nice rectangle $R \subset U_{p}$ for $(\Gamma)$ at $p$. From Lemma 3.5 we have that $\theta_{\Gamma}$ is constant on $(\Gamma) \cap R$. Covering $(\Gamma) \cap U_{p}$ with an appropriate countable family of nice rectangles, we obtain that $\theta_{\Gamma}$ is constant on $U_{p} \cap(\Gamma)$.

(a) Let $p \in \operatorname{Sing}_{\Gamma}$ and let $R$ be a nice rectangle for $(\Gamma)$ at $p$. Then (a) follows from (15) and the fact that every $\gamma_{i}$ that intersects $R(p)$ passes through $p$.

In the following we will refer to property (a) of Proposition 3.7 as to the upper semicontinuity of $\theta_{\Gamma}$.

Remark 3.8. The boundedness and the upper semicontinuity of $\theta_{\Gamma}$ have been proved, in different contexts, in [16].

Lemma 3.9. Let $\Gamma \in H^{2, p}(\mathbb{S})$ and $\widetilde{\Gamma} \in H^{2, p}(\widetilde{\mathbb{S}})$ be two equivalent systems of curves without crossings. Then

$$
\mathcal{F}(\Gamma)=\mathcal{F}(\widetilde{\Gamma}) .
$$

Proof. Let $p \in(\Gamma)$ and let $R=[-a, a] \times[-b, b]$ be a nice rectangle at $p$ for $(\Gamma)=(\widetilde{\Gamma})$. As proved in Lemma 3.5, we have

$$
\Gamma^{-1}(R)=\bigcup_{i=1}^{\theta_{\Gamma}(p)} I_{i}, \quad \widetilde{\Gamma}^{-1}(R)=\bigcup_{i=1}^{\theta_{\widetilde{\Gamma}}^{(p)}} \widetilde{I}_{i},
$$

where the $I_{i} \subset \mathbb{S}$ (respectively $I_{i} \subset \widetilde{\mathbb{S}}$ ) are open connected pairwise disjoint arcs. Furthermore, the image of each $I_{i}$ under $\Gamma$ (respectively of $\widetilde{I_{i}}$ under $\widetilde{\Gamma}$ ) is the graph of a function $f_{i}$ (respectively $\widetilde{f_{i}}$ ) of class $H^{2, p}$ passing through $p$ and the restriction of $\Gamma$ over $I_{i}$ (respectively of $\widetilde{\Gamma}$ over $\widetilde{I}_{i}$ ) is injective. Since $\Gamma$ and $\widetilde{\Gamma}$ are without crossings, using the locality of the weak derivatives in Sobolev spaces (see for instance [1, Proposition 3.71]), we have

$$
f_{i}^{\prime}=\tilde{f}_{j}^{\prime}, \quad f_{i}^{\prime \prime}=\widetilde{f}_{j}^{\prime \prime} \quad \text { a.e. on }\left\{f_{i}=f_{j}\right\}
$$

for every $i, j \in\left\{1, \ldots, \theta_{\Gamma}(p)=\theta_{\widetilde{\Gamma}}(p)\right\}$. Hence, as by hypothesis $(\Gamma) \cap R=(\widetilde{\Gamma}) \cap R$, we have

$$
\begin{aligned}
\mathcal{F}\left(\Gamma, \Gamma^{-1}(R)\right) & =\sum_{i=1}^{\theta_{\Gamma}(p)} \int_{-a, a[}\left(1+\frac{\left|f_{i}^{\prime \prime}\right|^{p}}{\left(1+\left(f_{i}^{\prime}\right)^{2}\right)^{3 p / 2}}\right) \sqrt{1+\left(f^{\prime}\right)^{2}} d x \\
& =\sum_{i=1}^{\theta_{\widetilde{\Gamma}}^{(p)}} \int_{]-a, a[}\left(1+\frac{\left|\widetilde{f}_{i}^{\prime \prime}\right|^{p}}{\left(1+\left(\widetilde{f}_{i}^{\prime}\right)^{2}\right)^{3 p / 2}}\right) \sqrt{1+\left(\widetilde{f}^{\prime}\right)^{2}} d x=\mathcal{F}\left(\widetilde{\Gamma}, \widetilde{\Gamma}^{-1}(R)\right) .
\end{aligned}
$$


Using Besicovitch Covering Theorem (see [12, Theorem 2.8.15]) we can find a countable family of nice rectangles $\left\{R\left(p_{n}\right)\right\}$ such that $\operatorname{int}\left(R\left(p_{n}\right)\right) \cap \operatorname{int}\left(R\left(p_{m}\right)\right)=\emptyset$ if $p_{n} \neq p_{m}$ and such that $\bigcup_{n \in \mathbb{N}} R\left(p_{n}\right)$ covers $\mathcal{H}^{1}$-almost all $(\Gamma)=(\widetilde{\Gamma})$. Using $(18)$ we have

$$
\mathcal{F}(\Gamma)=\sum_{n \in \mathbb{N}} \mathcal{F}\left(\Gamma, \Gamma^{-1}\left(R\left(p_{n}\right)\right)\right)=\sum_{n \in \mathbb{N}} \mathcal{F}\left(\widetilde{\Gamma}, \widetilde{\Gamma}^{-1}\left(R\left(p_{n}\right)\right)\right)=\mathcal{F}(\widetilde{\Gamma}),
$$

which is (17).

Remark 3.10. Using essentially the same proof, we can prove a local version of Lemma 3.9, that is: if $\Gamma \in H^{2, p}(\mathbb{S})$ and $\widetilde{\Gamma} \in H^{2, p}(\widetilde{\mathbb{S}})$ are two systems of curves which verify the hypothesis of Lemma 3.9 in $U \subset \mathbb{R}^{2}$, i.e., $(\Gamma) \cap U=(\widetilde{\Gamma}) \cap U$ and $\theta_{\Gamma}=\theta_{\widetilde{\Gamma}}$ on $(\Gamma) \cap U$, then

$$
\mathcal{F}\left(\Gamma, \Gamma^{-1}(U)\right)=\mathcal{F}\left(\widetilde{\Gamma}, \widetilde{\Gamma}^{-1}(U)\right) .
$$

Lemma 3.11. Let $\Gamma \in H^{2, p}(\mathbb{S})$ and $\widetilde{\Gamma} \in H^{2, p}(\widetilde{\mathbb{S}})$ be two systems of curves. If $\operatorname{Reg}_{\Gamma}=\operatorname{Reg}_{\widetilde{\Gamma}}$ and $\theta_{\Gamma}=\theta_{\widetilde{\Gamma}}$ on $\operatorname{Reg}_{\Gamma}$, then $\Gamma$ and $\widetilde{\Gamma}$ are equivalent.

Proof. From (13) we have

$$
(\widetilde{\Gamma})=\overline{\operatorname{Reg}_{\widetilde{\Gamma}}}=\overline{\operatorname{Reg}_{\Gamma}}=(\Gamma) .
$$

Therefore to prove that $\Gamma$ and $\widetilde{\Gamma}$ are equivalent it remains to check that $\theta_{\Gamma}=\theta_{\widetilde{\Gamma}}$ on $(\Gamma)$. Since by hypothesis $\theta_{\widetilde{\Gamma}}=\theta_{\Gamma}$ on $\operatorname{Reg}_{\widetilde{\Gamma}}=\operatorname{Reg}_{\Gamma}$ it is enough to check that $\theta_{\Gamma}=\theta_{\widetilde{\Gamma}}$ on $\operatorname{Sing}_{\widetilde{\Gamma}}=\operatorname{Sing}_{\Gamma}$. Let $p=0 \in \operatorname{Sing}_{\Gamma}$ and $R=[-a, a] \times[-b, b]$ be a nice rectangle for $(\widetilde{\Gamma})=(\Gamma)$ at $p$, such that $R$ verifies $(14)$, that is

$$
(\Gamma) \cap \partial R=(\widetilde{\Gamma}) \cap \partial R \subset \operatorname{Reg}_{\Gamma}=\operatorname{Reg}_{\tilde{\Gamma}} .
$$

Using Lemma 3.5 and the hypothesis $\theta_{\Gamma}=\theta_{\widetilde{\Gamma}}$ on $\operatorname{Reg}_{\Gamma}$ we have

$$
\theta_{\Gamma}(p)=\sum_{l=1}^{h} \theta_{\Gamma}\left(z_{l}\right)=\sum_{l=1}^{h} \theta_{\widetilde{\Gamma}}\left(z_{l}\right)=\theta_{\widetilde{\Gamma}}(p),
$$

which concludes the proof.

Lemma 3.12. Let $\Gamma$ be a system of curves without crossings, let $p \in(\Gamma)$ and $v$ be a unit vector normal to $(\Gamma)$ at $p$. Let $z_{1}:=p+t v, z_{2}:=p-t v$. Then $\mathcal{I}\left(\Gamma, z_{1}\right)+\mathcal{I}\left(\Gamma, z_{2}\right) \equiv \theta_{\Gamma}(p)(\bmod 2)$ for every $t>0$ small enough.

Proof. Using [4, Lemma 4.2] it follows that $\left|\mathcal{I}\left(\Gamma, z_{1}\right)-\mathcal{I}\left(\Gamma, z_{2}\right)\right|=|k-d|$, where $k, d \in \mathbb{N}$ are such that $k+d=$ $\theta_{\Gamma}(p)$. If $\mathcal{I}\left(\Gamma, z_{1}\right)+\mathcal{I}\left(\Gamma, z_{2}\right) \equiv 0(\bmod 2)$ then $\left|\mathcal{I}\left(\Gamma, z_{1}\right)-\mathcal{I}\left(\Gamma, z_{2}\right)\right|=|k-d|$ is even. Therefore $k$ and $d$ are either both odd or even, hence $\theta_{\Gamma}(p)$ is even, and $\mathcal{I}\left(\Gamma, z_{1}\right)+\mathcal{I}\left(\Gamma, z_{2}\right) \equiv \theta_{\Gamma}(p)(\bmod 2)$. If $\mathcal{I}\left(\Gamma, z_{1}\right)+\mathcal{I}\left(\Gamma, z_{2}\right) \equiv$ $1(\bmod 2)$ then $\left|\mathcal{I}\left(\Gamma, z_{1}\right)-\mathcal{I}\left(\Gamma, z_{2}\right)\right|$ is odd. Therefore $\theta_{\Gamma}(p)$ is odd, and $\mathcal{I}\left(\Gamma, z_{1}\right)+\mathcal{I}\left(\Gamma, z_{2}\right) \equiv \theta_{\Gamma}(p)(\bmod 2)$.

Using Lemma 3.12 we prove that given a system of curves $\Gamma$ without crossings, the set $\left\{q \in(\Gamma): \theta_{\Gamma}(q) \equiv\right.$ $1(\bmod 2)\}$ characterizes the set $A_{\Gamma}^{o}$ in $L^{1}\left(\mathbb{R}^{2}\right)$.

Proposition 3.13. Let $\Gamma \in H^{2, p}(\mathbb{S})$ and $\Lambda \in H^{2, p}(\widetilde{\mathbb{S}})$ be two systems of curves without crossings. Assume that

$$
\left\{q \in(\Gamma): \theta_{\Gamma}(q) \equiv 1(\bmod 2)\right\}=\left\{q \in(\Lambda): \theta_{\Lambda}(q) \equiv 1(\bmod 2)\right\} .
$$

Then

$$
\left|A_{\Gamma}^{o} \Delta A_{\Lambda}^{o}\right|=0
$$


Proof. Let $C$ be the closure of the set $\left\{q \in(\Gamma): \theta_{\Gamma}(q) \equiv 1(\bmod 2)\right\}$. We claim that $C=\partial\left(\operatorname{int}\left(A_{\Gamma}^{o} \cup(\Gamma)\right)\right)$. Using Lemma 3.12 it follows that $C \subseteq \partial\left(\operatorname{int}\left(A_{\Gamma}^{o} \cup(\Gamma)\right)\right)$. Now let $p \in \partial\left(\operatorname{int}\left(A_{\Gamma}^{o} \cup(\Gamma)\right)\right)$ and suppose that $p \notin C$. From the local constancy of the index it follows that $\partial\left(\operatorname{int}\left(A_{\Gamma}^{o} \cup(\Gamma)\right)\right) \subset(\Gamma)$, therefore $p \in(\Gamma)$. Since $p \notin C$, it follows that $\theta_{\Gamma}(q)$ is even for every $r>0$ small enough and every $q \in B_{r}(p) \cap(\Gamma)$. Using Lemma 3.12 we have that $\mathcal{I}(\Gamma, z)$ must be either always odd or always even for every $z \in B_{r}(p) \backslash(\Gamma)$ which contradicts the assumption $p \in \partial\left(\operatorname{int}\left(A_{\Gamma}^{o} \cup(\Gamma)\right)\right)$. Using (19) we have $C=\partial\left(\operatorname{int}\left(A_{\Gamma}^{o} \cup(\Gamma)\right)\right)=\partial\left(\operatorname{int}\left(A_{\Lambda}^{o} \cup(\Lambda)\right)\right)$. Let $z \notin(\Gamma)$ and let $\alpha$ be a continuous curve connecting $z$ with $\infty$ such that all the intersections between $(\Gamma) \cup(\Lambda)$ and $(\alpha)$ are transversal. Since $\mathcal{I}(\Gamma, z)(\bmod 2)$ (respectively $\mathcal{I}(\Lambda, z)(\bmod 2))$ can be computed using the parity of the number of the intersections of $(\Gamma)$ (respectively of $(\Lambda)$ ) with $(\alpha)$ and since $\operatorname{int}\left(A_{\Gamma}^{o} \cup(\Gamma)\right)$ and $\operatorname{int}\left(A_{\Lambda}^{o} \cup(\Lambda)\right.$ ) are two bounded open subsets of $\mathbb{R}^{2}$ with the same boundary we have $\operatorname{int}\left(A_{\Gamma}^{o} \cup(\Gamma)\right)=\operatorname{int}\left(A_{\Lambda}^{o} \cup(\Lambda)\right)$. Therefore $A_{\Gamma}^{o} \Delta A_{\Lambda}^{o} \subseteq(\Gamma) \cup(\Lambda)$, so that $\left|A_{\Gamma}^{o} \Delta A_{\Lambda}^{o}\right| \leqslant|(\Gamma) \cup(\Lambda)|=0$.

\section{Preliminary lemmata}

In this section we prove some lemmata needed in the proof of Theorems 5.1, 6.3, 7.1.

Lemma 4.1. Let $\alpha, \beta:[0,1] \mapsto \mathbb{R}^{2}$ be two regular curves of class $H^{2, p}$ such that $\alpha(1)=\beta(0)$ and $\alpha^{\prime}(1)$ is parallel to $\beta^{\prime}(0)$. Then there is a regular curve $\gamma:[0,1] \mapsto \mathbb{R}^{2}$ of class $H^{2, p}$ such that $(\gamma)=(\alpha) \cup(\beta)$ and $\left|\gamma^{\prime}\right|$ is constant on $[0,1]$.

Proof. Let $\alpha:[0, l(\alpha)] \mapsto \mathbb{R}^{2}$ (respectively $\beta:[0, l(\beta)] \mapsto \mathbb{R}^{2}$ ) be the reparametrization of $\alpha$ (respectively of $\beta$ ) by arc length such that $\alpha(l(\alpha))=\beta(0)$ and $\dot{\alpha}(l(\alpha))=\dot{\beta}(0)$. Define $\gamma:[0, l(\alpha)+l(\beta)] \mapsto \mathbb{R}^{2}$ by

$$
\gamma(s):= \begin{cases}\alpha(s) & \text { if } s \in[0, l(\alpha)] \\ \beta(s-l(\alpha)) & \text { if } s \in] l(\alpha), l(\alpha)+l(\beta)] .\end{cases}
$$

Since $\alpha$ and $\beta$ are regular curves of class $\mathcal{C}^{1}$ and $\alpha(l(\alpha))=\beta(0), \dot{\alpha}(l(\alpha))=\dot{\beta}(0)$, then $\gamma$ is a regular curve of class $\mathcal{C}^{1}$ and $\dot{\gamma}=\dot{\alpha}$ (respectively $\dot{\gamma}=\dot{\beta}$ ) on $[0, l(\alpha)]$ (respectively on $[l(\alpha), l(\alpha)+l(\beta)]$ ). Using two integrations by parts and the assumptions on $\alpha$ and $\beta$ one checks that the second distributional derivative $\ddot{\gamma}$ of $\gamma$ is represented by an $L^{p}$ function and $\ddot{\gamma}=\ddot{\alpha}$ (respectively $\ddot{\gamma}=\ddot{\beta}$ ) almost everywhere on $] 0, l(\alpha)$ [ (respectively almost everywhere on $] l(\alpha), l(\alpha)+l(\beta)[)$. Reparametrizing $\gamma$ with $t:=s /(l(\alpha)+l(\beta))$ we obtain the thesis.

Definition 4.2. Let $a>0$ and $\left\{g_{1}, \ldots, g_{r}\right\}$ be a finite family of functions in $\mathcal{C}^{1}([0, a])$. We say that $\operatorname{graph}\left(g_{1}\right), \ldots, \operatorname{graph}\left(g_{r}\right)$ meet tangentially in $[0, a]$ if given $j, l \in\{1, \ldots, r\}$ and $x \in[0, a]$ such that $g_{j}(x)=g_{l}(x)$, then $g_{j}^{\prime}(x)=g_{l}^{\prime}(x)$. We say that $\operatorname{graph}\left(g_{1}\right), \ldots, \operatorname{graph}\left(g_{r}\right)$ pass through zero horizontally if $g_{j}(0)=g_{j}^{\prime}(0)=0$ for any $j \in\{1, \ldots, r\}$.

Lemma 4.3. Let $a>0$ and $f_{1}, \ldots, f_{r}$ be a family of distinct functions of class $H^{2, p}(] 0, a[)$ whose graphs meet tangentially in $[0, a]$ and pass through zero horizontally. Define

$$
\Sigma:=\left\{g \in \mathcal{C}^{0}([0, a]): \operatorname{graph}(g) \subseteq \bigcup_{l=1}^{r} \operatorname{graph}\left(f_{l}\right)\right\} .
$$

Then $\Sigma$ is a bounded subset of $H^{2, p}(] 0, a[)$.

Remark 4.4. The fact that $\Sigma$ is a bounded subset of $\mathcal{C}^{1}([0, a])$ was already observed in the proof of Theorem 6.4 of [4]. 
Proof. Let $g \in \Sigma$. Assume first that $[0, a]=\bigcup_{i=1}^{d}\left[\alpha_{i}, \beta_{i}\right]$, with $d>0$ a natural number and $\left[\alpha_{i}, \beta_{i}\right]$ intervals (with $0=: \alpha_{1}<\beta_{1}<\alpha_{2}<\cdots<\beta_{d-1}<\alpha_{d}<\beta_{d}:=a$ ) where $g$ is equal to some $f_{l_{i}}$. Let $\varphi \in \mathcal{C}_{c}^{\infty}$ (]0, $a$ [). By Remark 4.4 we have that $g \in \mathcal{C}^{1}([0, a])$, therefore

$$
\begin{aligned}
\int_{] 0, a[} g^{\prime} \varphi^{\prime} d x & =\sum_{i=1}^{d} \int_{] \alpha_{i}, \beta_{i}[} f_{l_{i}}^{\prime} \varphi^{\prime} d x \\
& =\sum_{i=1}^{d}\left[-\int_{] \alpha_{i}, \beta_{i}[} f_{l_{i}}^{\prime \prime} \varphi d x+\varphi\left(\beta_{i}\right) f_{l_{i}}^{\prime}\left(\beta_{i}\right)-\varphi\left(\alpha_{i}\right) f_{l_{i}}^{\prime}\left(\alpha_{i}\right)\right]=-\sum_{i=1}^{d} \int_{] \alpha_{i}, \beta_{i}[} f_{l_{i}}^{\prime \prime} \varphi d x,
\end{aligned}
$$

where we used the fact that the $f_{j}$ meet tangentially and the compactness of the support of $\varphi$. Using (21) it follows that $g \in H^{2, p}(] 0, a[)$ and

$$
\left\|g^{\prime \prime}\right\|_{L^{p}(] 0, a[)} \leqslant \sum_{l=1}^{r}\left\|f_{l}^{\prime \prime}\right\|_{L^{p}(] 0, a[)} .
$$

Using (22) and the fact that $\Sigma$ is a bounded subset of $\mathcal{C}^{1}([0, a])$ (Remark 4.4) we deduce that

$$
\|g\|_{H^{2, p}(] 0, a[)} \leqslant C,
$$

where $C>0$ is a constant independent of $g$.

Assume now that $g \in \Sigma$ is arbitrary. Fix a dense countable subset $D=\left\{x^{k}\right\}$ of $[0, a]$. We want to approximate $g$ in the weak topology of $H^{2, p}(] 0, a$ [) with a sequence $\left\{g_{n}\right\} \subset \Sigma$ such that $g_{n}\left(x^{k}\right)=g\left(x^{k}\right)$ for every $k=1, \ldots, n$ and each $g_{n}$ satisfies the hypothesis of the preceding step. To construct $\left\{g_{n}\right\}$ we proceed in the following way. Fix $n \in \mathbb{N}$ and relabel the first $n$ elements of $D$ in such a way that $x^{0}:=0<x^{1}<\cdots<x^{n}<x^{n+1}:=a$. We can also assume that $\bigcup_{n \in \mathbb{N}}\left\{x^{0}, \ldots, x^{n+1}\right\}=D$. We give the definition of $g_{n}$ over each interval $\left[x^{h}, x^{h+1}\right]$. Let $h \in\{0, \ldots, n\}$. We have two cases.

Case 1 . There exists $l \in\{1, \ldots, r\}$ such that $f_{l}\left(x^{h}\right)=g\left(x^{h}\right)$ and $f_{l}\left(x^{h+1}\right)=g\left(x^{h+1}\right)$. In this case we set $g_{n}:=f_{l}$ on $\left[x^{h}, x^{h+1}\right]$.

Case 2. For every $l \in\{1, \ldots, r\}$ either $f_{l}\left(x^{h}\right) \neq g\left(x^{h}\right)$ or $f_{l}\left(x^{h+1}\right) \neq g\left(x^{h+1}\right)$. Define

$$
\xi^{1}:=\inf \{x \in] x^{h}, x^{h+1}\left[: \exists l \in\{1, \ldots, r\}: f_{l}(x)=g(x) \text { and } f_{l}\left(x^{h+1}\right)=g\left(x^{h+1}\right)\right\} .
$$

Notice that $x^{h}<\xi^{1}$, otherwise we are in case 1 ; moreover, the fact that $g$ is continuous and its graphs is contained in the union of the graphs of the $f_{i}$ imply that $\xi^{1}<x^{h+1}$. Finally, there is $l_{1} \in\{1, \ldots, r\}$ such that $f_{l_{1}}\left(\xi^{1}\right)=g\left(\xi^{1}\right)$ and $f_{l_{1}}\left(x^{h+1}\right)=g\left(x^{h+1}\right)$. We set $g_{n}:=f_{l_{1}}$ on $\left[\xi^{1}, x^{h+1}\right]$. Now, if there is $l \in\{1, \ldots, r\}$ such that $f_{l}\left(x^{h}\right)=g\left(x^{h}\right)$ and $f_{l}\left(\xi^{1}\right)=g\left(\xi^{1}\right)$ we set $g_{n}:=f_{l}$ on $\left[x^{h}, \xi^{1}\right]$ and the algorithm stops. Otherwise we define

$$
\xi^{2}:=\inf \{x \in] x^{h}, \xi^{1}\left[: \exists l \in\{1, \ldots, r\}: f_{l}(x)=g(x) \text { and } f_{l}\left(\xi^{1}\right)=g\left(\xi^{1}\right)\right\},
$$

and set $g_{n}:=f_{l_{2}}$ on $\left[\xi^{2}, \xi^{1}\right]$, where $l_{2} \in\{1, \ldots, r\}$ is such that $f_{l_{2}}\left(\xi^{2}\right)=g\left(\xi^{2}\right)$ and $f_{l_{2}}\left(\xi^{1}\right)=g\left(\xi^{1}\right)$.

Repeating the same argument $i$-times, $i$ an arbitrary natural number, the function $g_{n}$ is defined on $\left[\xi^{i}, x^{h+1}\right] \subseteq$ $\left[x^{h}, x^{h+1}\right]$ and $g_{n}$ agrees with one of the $f_{l}$ on each interval $\left[\xi^{j}, \xi^{j-1}\right]$, with $j=1, \ldots, i$.

Observe that, if for some $j \in\{1, \ldots, i\}$ and $l \in\{1, \ldots, r\}$ we have $f_{l}\left(\xi^{j}\right)=g\left(\xi^{j}\right)$ then, by definition of $\xi^{j}$, $f_{l}(x) \neq g(x)$ for every $x \in\left[x^{h}, \xi^{j}\right]$. Since we deal with $r$ distinct functions, after a finite number $K \leqslant r$ of steps, necessarily there is $l_{K} \in\{1, \ldots, r\}$ such that $f_{l_{K}}\left(x^{h}\right)=g\left(x^{h}\right)$ and $f_{l_{K}}\left(\xi^{K}\right)=g\left(\xi^{K}\right)$. Setting $g_{n}:=f_{l_{K}}$ on $\left[x^{h}, \xi^{K}\right]$, we obtain that there is a finite number of closed intervals (with pairwise disjoint interior), whose union is the whole interval $\left[x^{h}, x^{h+1}\right]$, where $g_{n}$ agrees with one of the $f_{l}$.

Now, repeating this construction for every $h=1, \ldots, n$, we obtain the desired function $g_{n}$.

Recalling (22), we have that the $H^{2, p}$ norm of $g_{n}$ is uniformly bounded with respect to $n$. It follows that $\left\{g_{n}\right\}$ has a subsequence that converges weakly in $H^{2, p}(] 0, a[)$ to a certain $\widehat{g} \in H^{2, p}(] 0, a[)$. Since $H^{2, p}$ weak convergence 
implies uniform convergence, we have that $g$ and $\widehat{g}$ coincide on the dense set $D$, hence $g \equiv \widehat{g}$ on $[0, a]$. Therefore $g \in H^{2, p}(] 0, a[)$ and (23) holds.

Given an open interval $I$ and a function $g \in H^{2, p}(I)$, we define

$$
\mathcal{P}(g):=\int_{I}\left[1+\left(\frac{\left|g^{\prime \prime}\right|}{\left(1+\left(g^{\prime}\right)^{2}\right)^{3 / 2}}\right)^{p}\right] \sqrt{1+\left(g^{\prime}\right)^{2}} d x .
$$

As proved in [4], $\mathcal{P}(g)$ equals the energy $\mathcal{F}(\gamma)$ of a simple curve $\gamma$ whose support is the graph of $g$. The next lemma is concerned with $\mathcal{P}$-minimal connections between the origin and a given point $z_{j}$, see also Fig. 6 .

Lemma 4.5. Let $a, f_{1}, \ldots, f_{r}$ and $\Sigma$ be as in Lemma 4.3. Set

$$
\left\{z_{1}, \ldots, z_{h}\right\}=\left\{\left(a, f_{1}(a)\right), \ldots,\left(a, f_{r}(a)\right)\right\}
$$

(observe that in general $h \leqslant r$ ). Let

$$
\Sigma_{j}:=\left\{g \in \Sigma: g(a)=z_{j}\right\}, \quad j \in\{1, \ldots, h\} .
$$

Then the problem

$$
\min \left\{\mathcal{P}(g,] 0, a[): g \in \Sigma_{j}\right\}
$$

admits a solution. Moreover if $j \neq l$, there exist a minimizer $g_{j}$ of $\mathcal{P}$ over $\Sigma_{j}$ and a minimizer $g_{l}$ of $\mathcal{P}$ over $\Sigma_{l}$ such that the following property holds: iffor some $c \in] 0, a\left[\right.$ we have $g_{j}(c)=g_{l}(c)$, then $g_{j} \equiv g_{l}$ on $[0, c]$.

Proof. The weak $H^{2, p}$ sequential lower semicontinuity of the functional $\mathcal{P}(\cdot] 0,, a[)$ follows from $[9$, Theorem 3.4, p. 74]. Using Lemma 4.3 it follows that $\Sigma_{j}$ is $H^{2, p}$-weakly compact for every $j \in\{1, \ldots, h\}$. Therefore the minimum problem (25) admits a solution.
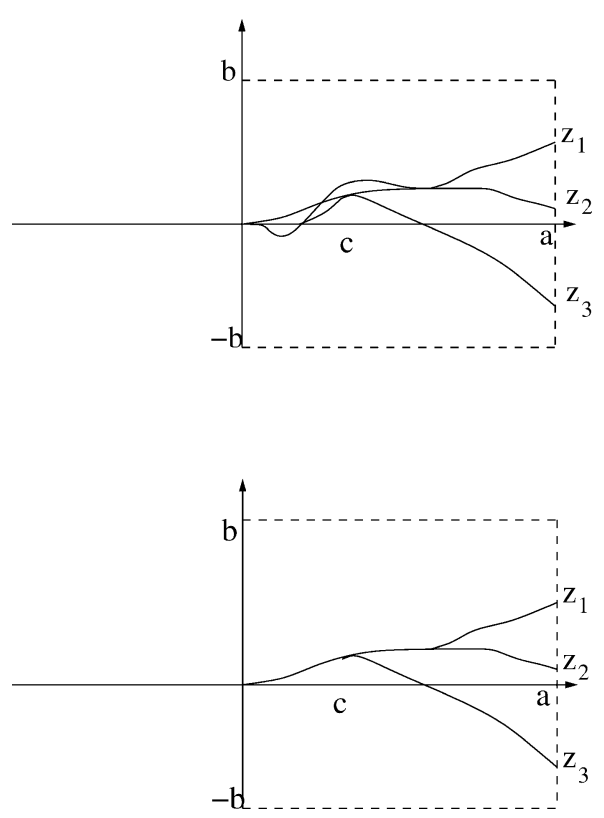

Fig. 6. These two figures show the construction in the proof of Lemma 4.5. In the first figure we depict three solutions $g_{j}, j=1,2,3$ of the minimum problem (25), and $g_{2}(c)=g_{3}(c)$. In the second figure we depict the resulting minimizers: in this case $g_{2} \equiv g_{3}$ on $[0, c]$. 
Let us fix $g_{1} \in \Sigma_{1}$ solution of (25) for $j=1$. Take a function $\widehat{g}_{2} \in \Sigma_{2}$ solution of (25) for $j=2$ and let

$$
s:=\sup \left\{x \in[0, a]: g_{1}(x)=\widehat{g}_{2}(x)\right\} .
$$

Clearly $s<a$. If $s=0$ then the graphs of $g_{1}$ and $\widehat{g}_{2}$ meet only at 0 and in this case we set $g_{2}:=\widehat{g}_{2}$. Suppose $s>0$. Note that

$$
\mathcal{P}\left(g_{1},\right] 0, s[)=\mathcal{P}\left(\widehat{g}_{2},\right] 0, s[) .
$$

Indeed, if not, assuming by contradiction for instance that $\mathcal{P}\left(g_{1},\right] 0, s[)>\mathcal{P}\left(\widehat{g}_{2},\right] 0, s[)$, we can define the function

$$
\widehat{g}_{1}:= \begin{cases}\widehat{g}_{2} & \text { on }[0, s] \\ g_{1} & \text { on }] s, a] .\end{cases}
$$

Using also Lemma 4.1 we have $\widehat{g}_{1} \in \Sigma_{1}$; moreover $\mathcal{P}\left(g_{1},\right] 0, a[)>\mathcal{P}\left(\widehat{g}_{2},\right] 0, a[)$, thus contradicting the minimality of $g_{1}$ over $\Sigma_{1}$.

Now we define

$$
g_{2}:= \begin{cases}g_{1} & \text { on }[0, s] \\ \widehat{g}_{2} & \text { on }] s, a]\end{cases}
$$

By (26) we have $\mathcal{P}\left(\widehat{g_{2}},\right] 0, a[)=\mathcal{P}\left(g_{2},\right] 0, a[)$. Therefore $g_{2}$ is still a minimizer of $\mathcal{P}(\cdot] 0,, a[)$ over $\Sigma_{2}$. Now take a function $\widehat{g}_{3} \in \Sigma_{3}$ solution of (25) for $j=3$, define

$$
\sigma:=\sup \left\{x \in[0, a]: \text { either } \widehat{g}_{3}(x)=g_{2}(x) \text { or } \widehat{g}_{3}(x)=g_{1}(x)\right\},
$$

and make the same operation above to obtain the function $g_{3}$.

Repeating the same argument for each $z_{j}$ we obtain a family of minimizers of $\mathcal{P}$ satisfying the required properties.

Remark 4.6. The last assertion concerning $g_{j}$ and $g_{l}$ in Lemma 4.5 is crucial in the proof of Theorem 5.1, since it allows to locally modify an arbitrary $H^{2, p}$ system of curves into a new system verifying the finiteness property.

\subsection{Finite unions of graphs, generalized multiplicity, canonical families}

Definition 4.7. Let $r \in \mathbb{N} \backslash\{0\}, I \subset \mathbb{R}$ a closed interval and

$$
Y:=\left\{\left(g_{1}, \mu_{1}\right), \ldots,\left(g_{r}, \mu_{r}\right)\right\}
$$

be a family of pairs where $g_{l}: I \rightarrow \mathbb{R}$ is a continuous function and $\mu_{l} \in \mathbb{N} \backslash\{0\}$ for every $l=1, \ldots, r$. We set

$$
\operatorname{graph}(Y):=\bigcup_{i=1}^{r} \operatorname{graph}\left(g_{i}\right) .
$$

We call the function

$$
\eta_{Y}: \operatorname{graph}(Y) \mapsto \mathbb{N} \backslash\{0\}, \quad \eta_{Y}(x, y):=\sum_{l: g_{l}(x)=y} \mu_{l},
$$

the generalized multiplicity of $Y$.

Remark 4.8. Let $b$ be a real number with $b>\max _{1 \leqslant l \leqslant r}\left\|g_{l}\right\|_{L^{\infty}(] 0, a[)}$. Then

$$
\sum_{z \in(\{x\} \times[-b, b]) \cap \operatorname{graph}(Y)} \eta_{Y}(z)=\sum_{l=1}^{r} \mu_{l} \quad \forall x \in[0, a] .
$$

Clearly if all $g_{i}(x)$ have the same value at $x=0$, then $\eta_{Y}(0)=\sum_{l=1}^{r} \mu_{l}$ for any $x \in[0, a]$. 
In the following lemma we do not assume that the union of the graphs of the functions $g_{j}$ (in $R^{+}$) is contained in $(\Gamma) \cap R^{+}$.

Lemma 4.9. Let $\Gamma \in H^{2, p}(\mathbb{S})$ be a system of curves without crossings. Let $p=0 \in(\Gamma), R=[-a, a] \times[-b, b]$ be a nice rectangle for $(\Gamma)$ at $p$ and set

$$
\left\{z_{1}, \ldots, z_{h}\right\}=(\Gamma) \cap(\{a\} \times[-b, b]) .
$$

Let $s \in \mathbb{N}, s \geqslant h$, let $\left\{g_{1}, \ldots, g_{s}\right\} \subset H^{2, p}(] 0, a[)$ be a collection of distinct functions, $\left\{\mu_{1}, \ldots, \mu_{s}\right\} \subset \mathbb{N} \backslash\{0\}$, and $Y:=\left\{\left(g_{1}, \mu_{1}\right), \ldots,\left(g_{s}, \mu_{s}\right)\right\}$. Assume that

- $\operatorname{graph}\left(g_{1}\right), \ldots, \operatorname{graph}\left(g_{s}\right)$ meet tangentially in $[0, a]$ and pass through zero horizontally;

- $\left\{\left(a, g_{1}(a)\right), \ldots,\left(a, g_{s}(a)\right)\right\}=\left\{z_{1}, \ldots, z_{h}\right\}$

- if $\left(a, g_{l}(a)\right)=z_{j}$ for some $l \in\{1, \ldots, s\}$ and $j \in\{1, \ldots, h\}$, then the vector $\left(1, g_{l}^{\prime}(a)\right)$ is parallel to $T_{z_{j}}(\Gamma)$;

$-\sum_{l=1}^{s} \mu_{l}=\theta_{\Gamma}(p)$.

If the generalized multiplicity $\eta_{Y}$ of $Y$ satisfies

$$
\eta_{Y}\left(z_{j}\right)=\theta_{\Gamma}\left(z_{j}\right) \quad \forall j \in\{1, \ldots, h\}
$$

then there exists a system of curves $\Lambda \in H^{2, p}(\mathbb{S})$, having the same number of curves as $\Gamma$, with the following properties:

$(\Lambda) \cap R^{+}=\operatorname{graph}(Y)$ and $\theta_{\Lambda}=\eta_{Y} \quad$ on $\operatorname{graph}(Y)$;

$(\Lambda) \backslash R^{+}=(\Gamma) \backslash R^{+}$and $\theta_{\Lambda}=\theta_{\Gamma} \quad$ out of $R^{+}$.

Proof. Let $\Gamma=\left\{\gamma_{1}, \ldots, \gamma_{m}\right\}$. As observed in the proof of Lemma 3.5, we have

$$
\Gamma^{-1}\left(R^{+}\right)=\bigcup_{i=1}^{m} \gamma_{i}^{-1}\left(R^{+}\right)=\bigcup_{i=1}^{m} \bigcup_{k=1}^{\sharp\left\{\gamma_{i}^{-1}(p)\right\}} I_{i k},
$$

where $\theta_{\Gamma}(p)=\sum_{i=1}^{m} \sharp\left\{\gamma_{i}^{-1}(p)\right\}$ and $I_{i k}$ are closed, connected, pairwise disjoint arcs of $S_{i}^{1}$. Fix $j \in\{1, \ldots, h\}$. Using (28) and (27) we have $\sharp\left\{I_{i k}: z_{j} \in \gamma_{i}\left(I_{i k}\right)\right\}=\theta_{\Gamma}\left(z_{j}\right)=\eta_{Y}\left(z_{j}\right)=\sum_{l:\left(a, g_{l}(a)\right)=z_{j}} \mu_{l}$. Write $I_{i k}=\left(s_{1}, s_{2}\right)$. As, for any $k=1, \ldots, m$, the first components of the two vectors $\gamma_{i}^{\prime}\left(s_{1}\right), \gamma_{i}^{\prime}\left(s_{2}\right)$ are either both positive or both negative, we can apply Lemma 4.1 and obtain a new $H^{2, p}$ curve whose image in $R^{+}$is given by $\operatorname{graph}\left(g_{l}\right)$ for some $l$ such that $\left(a, g_{l}(a)\right)=z_{j}$. Fixed $z_{j}$ we repeat the same argument for every $I_{i k}$ such that $z_{j} \in \gamma_{i}\left(I_{i k}\right)$ in such a way that, for every $l \in\{1, \ldots, s\}$ such that $\left(a, g_{l}(a)\right)=z_{j}$, $\operatorname{graph}\left(g_{l}\right)$ is parametrized exactly $\mu_{l}$ times. Repeating this construction for every $j \in\{1, \ldots, h\}$ we obtain the new system of curves $\Lambda$.

Definition 4.10. Let $\Gamma \in H^{2, p}(\mathbb{S})$ be a system of curves without crossings. Let $p=0 \in(\Gamma)$ and $R=[-a, a] \times$ $[-b, b]$ be a nice rectangle for $(\Gamma)$ at $p$. Let $\left\{f_{1}, \ldots, f_{r}\right\} \subset H^{2, p}(]-a, a[)$ be a collection of distinct functions and $\left\{\mu_{1}, \ldots, \mu_{r}\right\} \subset \mathbb{N} \backslash\{0\}$. We say that $Y:=\left\{\left(f_{1}, \mu_{1}\right), \ldots,\left(f_{r}, \mu_{r}\right)\right\}$ is a canonical family for $(\Gamma)$ in $R$ if

$$
(\Gamma) \cap R=\operatorname{graph}(Y) \cap R \quad \text { and } \quad \theta_{\Gamma}=\eta_{Y} \quad \text { on }(\Gamma) \cap R .
$$

Lemma 4.11. Let $\Gamma$ and $R$ be as in Definition 4.10. Then there exists a canonical family $Y$ for $(\Gamma)$ in $R$.

Proof. Since $\theta_{\Gamma}$ takes nonnegative integer values, we can consider $\mu_{1}:=\min \left\{\theta_{\Gamma}(q): q \in R\right\} \in \mathbb{N} \backslash\{0\}$. From (16) and (13), it follows that we can find $q_{1} \in \operatorname{Reg}_{\Gamma} \cap R$ such that $\theta_{\Gamma}\left(q_{1}\right)=\mu_{1}$. From (b) in Proposition 3.7, it follows that $\theta_{\Gamma} \equiv \mu_{1}$ on a whole connected component $C_{1}$ of $\operatorname{Reg}_{\Gamma} \cap R$ containing $q_{1}$. Now let $f_{1} \in H^{2, p}(]-a, a[)$ be 
such that $C_{1} \subseteq \operatorname{graph}\left(f_{1}\right) \subseteq(\Gamma) \cap R$ (the existence of $f_{1}$ is ensured from the fact that $R$ is a nice rectangle for $(\Gamma))$. Then consider the function

$$
\Psi_{1}:(\Gamma) \cap R \rightarrow \mathbb{N}, \quad \Psi_{1}:= \begin{cases}\theta_{\Gamma}-\mu_{1} & \text { on } \operatorname{graph}\left(f_{1}\right), \\ \theta_{\Gamma} & \text { otherwise on } R,\end{cases}
$$

and define $\mathcal{G}_{1}:=\left\{q \in \operatorname{int}(R): \Psi_{1}(q)>0\right\}$. As $\theta_{\Gamma}$ verifies (15) we have that $\Psi_{1}$ verifies the train tracks property in $\operatorname{int}(R)$. Now, observing that $\mathcal{G}_{1}$ is still a finite union of $H^{2, p}$ graphs, that the train tracks and the upper semicontinuity properties still hold for $\mathcal{G}_{1}$ and that $\operatorname{Sing}_{\Gamma} \supseteq \operatorname{Sing}_{\mathcal{G}_{1}}$, we repeat the argument above replacing $(\Gamma)$ with $\mathcal{G}_{1}$ and $\theta_{\Gamma}$ with $\Psi_{1}$. In this way we obtain $\mu_{2}:=\min \left\{\Psi_{1}(q): q \in R\right\} \in \mathbb{N} \backslash\{0\}$, a connected component $C_{2}$ of $\operatorname{Reg}_{\Gamma} \cap \mathcal{G}_{1}$ and a function $f_{2} \in H^{2, p}(]-a, a[)$ such that $C_{2} \subseteq \operatorname{graph}\left(f_{2}\right) \subseteq \mathcal{G}_{1} \subseteq(\Gamma)$. Repeating this construction, after $r \leqslant \theta_{\Gamma}(p)$ steps, we obtain that $\mathcal{G}_{r+1}=\emptyset$. In this way we construct a family $Y:=\left\{\left(f_{1}, \mu_{1}\right), \ldots,\left(f_{r}, \mu_{r}\right)\right\}$ such that

$$
f_{l} \neq f_{j} \text { for every } l \neq j \text {, since if } l<j \text { then } \operatorname{graph}\left(f_{j}\right) \cap C_{l}=\emptyset \text { and } C_{l} \subset \operatorname{graph}\left(f_{l}\right),
$$

and satisfying (29).

We conclude this section by observing that the definition of canonical family for a system of curves could be related with the notion of $\mathcal{C}^{1, \alpha}$ multiple function appearing in varifolds theory, see for instance [16].

\section{Main result on the approximation of systems of curves}

The following theorem is the crucial approximation result for systems of curves of class $H^{2, p}$, and is one of the main results of the paper.

Theorem 5.1. Let $\Gamma$ be a system of curves of class $H^{2, p}(\mathbb{S})$ without crossings. Then there exist a parameter space $\widetilde{\mathbb{S}}$, a limit system of curves $\widetilde{\Gamma} \in H^{2, p}(\widetilde{\mathbb{S}})$ equivalent to $\Gamma$ and a sequence $\left\{\widetilde{\Gamma}_{N}\right\}$ of limit systems of curves of class $H^{2, p}(\widetilde{\mathbb{S}})$ satisfying the finiteness property, such that

$$
\widetilde{\Gamma}_{N} \rightarrow \widetilde{\Gamma} \quad \text { weakly in } H^{2, p}(\widetilde{\mathbb{S}}), \quad \lim _{N \rightarrow \infty} \mathcal{F}\left(\widetilde{\Gamma}_{N}\right)=\mathcal{F}(\Gamma),
$$

and

$$
\left(\widetilde{\Gamma}_{N}\right) \subseteq(\Gamma), \quad \mathcal{F}\left(\widetilde{\Gamma}_{N}\right) \leqslant \mathcal{F}(\Gamma) \quad \forall N \in \mathbb{N} .
$$

Proof. The proof is divided into three steps.

Step 1 . We construct a sequence $\left\{\Lambda_{N}\right\} \subset H^{2, p}(\mathbb{S})$ of systems of curves (not necessarily limit systems of curves) such that, for every $N \in \mathbb{N}$, the following properties hold:

$-\left(\Lambda_{N}\right) \subseteq(\Gamma)$

$-\Lambda_{N}$ verifies the finiteness property;

$-\mathcal{F}\left(\Lambda_{N}\right) \leqslant \mathcal{F}(\Gamma)$.

Fix $N \in \mathbb{N}$. For any $p \in \operatorname{Sing}_{\Gamma}$ let $R(p)$ be a nice rectangle for $\Gamma$ centered at $p$, with diameter strictly smaller than $2^{-N}$. By (12) the set $\operatorname{Sing}_{\Gamma}$ is compact, hence there are $p_{1}, \ldots, p_{m(N)}$ points of $\operatorname{Sing}_{\Gamma}$ such that

$$
\operatorname{Sing}_{\Gamma} \subset \bigcup_{i=1}^{m(N)} R\left(p_{i}\right) .
$$


Recalling (14), we can assume that $(\Gamma) \cap \partial R\left(p_{i}\right) \subset \operatorname{Reg}_{\Gamma}$ for any $i \in\{1, \ldots, m(N)\}$. In order to construct the system $\Lambda_{N}$ we use a recursive algorithm consisting of $m(N)$ steps. We proceed as follows: let $\Lambda_{0}^{N}:=\Gamma$, let $1 \leqslant i \leqslant m(N)$, and suppose that $\Lambda_{i-1}^{N}$ has been defined. Then $\Lambda_{i}^{N}$ is obtained by modifying $\Lambda_{i-1}^{N}$ only on $\operatorname{int}\left(R\left(p_{i}\right)\right)$, in particular $\left(\Lambda_{i}^{N}\right) \backslash R\left(p_{i}\right)=\left(\Lambda_{i-1}^{N}\right) \backslash R\left(p_{i}\right)$, in such a way that:

(i) $\left(\Lambda_{i}^{N}\right) \subseteq\left(\Lambda_{i-1}^{N}\right)$;

(ii) $\Lambda_{i}^{N}$ verifies the finiteness property in $\operatorname{int}\left(R\left(p_{i}\right)\right)$;

(iii) $\mathcal{F}\left(\Lambda_{i}^{N}\right) \leqslant \mathcal{F}\left(\Lambda_{i-1}^{N}\right)$;

(iv) $\Lambda_{i}^{N}$ and $\Lambda_{i-1}^{N}$ are defined on the same parameter space.

Let us define $\Lambda_{i}^{N}$. To simplify the notation, we assume that $p_{i}=0$, that $T_{p_{i}}\left(\Lambda_{i-1}^{N}\right)$ coincides with the $x$-axis and that $R\left(p_{i}\right)=[-a, a] \times[-b, b]$. We shall work on $\left(\Lambda_{i-1}^{N}\right) \cap R^{+}\left(p_{i}\right)$, since the modification of $\Lambda_{i-1}^{N}$ on the set $\left(\Lambda_{i-1}^{N}\right) \cap R^{-}\left(p_{i}\right)$ is similar. Because of the assumptions on $R\left(p_{i}\right)$ and the inclusion $\left(\Lambda_{i-1}^{N}\right) \subseteq(\Gamma)$, we have that $\left(\Lambda_{i-1}^{N}\right) \cap(\{a\} \times[-b, b])$ consists of a finite set of distinct points $z_{1}^{+}, \ldots, z_{h}^{+}$, labelled by their $y$-coordinate. Let $\left\{f_{1}^{+}, \ldots, f_{r^{+}}^{+}\right\} \subset H^{2, p}(] 0, a[)$ be the family of distinct functions such that $\left(\Lambda_{i-1}^{N}\right) \cap R^{+}\left(p_{i}\right)=\bigcup_{l=1}^{r^{+}} \operatorname{graph}\left(f_{l}^{+}\right)$.

For any $j \in\{1, \ldots, h\}$ let

$$
\Sigma_{j}^{+}:=\left\{g \in \mathcal{C}^{0}([0, a]): \operatorname{graph}(g) \subseteq\left(\Lambda_{i-1}^{N}\right) \cap R^{+}\left(p_{i}\right), g(a)=z_{j}^{+}\right\} .
$$

Consider the problem

$$
\min \left\{\mathcal{P}(g,] 0, a[): g \in \Sigma_{j}^{+}\right\},
$$

where $\mathcal{P}$ is defined in (24). According to Lemma 4.5, for every $j=1, \ldots, h$ we can select a function $g_{j}^{+} \in \Sigma_{j}^{+}$, minimum of $\mathcal{P}$ over $\Sigma_{j}^{+}$, such that, if $j \neq l$ and if for some $\left.c \in\right] 0, a$ [ we have $g_{j}^{+}(c)=g_{l}^{+}(c)$, then $g_{j}^{+} \equiv g_{l}^{+}$on $[0, c]$. Then we replace all the $f_{1}^{+}, \ldots, f_{r^{+}}^{+}$with the $g_{1}^{+}, \ldots, g_{h}^{+}$. Observe that $\bigcup_{k=1}^{h} \operatorname{graph}\left(g_{k}^{+}\right) \subseteq \bigcup_{l=1}^{r^{+}} \operatorname{graph}\left(f_{l}^{+}\right)$. Now consider the family $Y^{+}:=\left\{\left(g_{1}^{+}, \theta_{\Lambda_{i-1}^{N}}\left(z_{1}^{+}\right)\right), \ldots,\left(g_{h}^{+}, \theta_{\Lambda_{i-1}^{N}}\left(z_{h}^{+}\right)\right)\right\}$and let $\eta_{Y^{+}}: \operatorname{graph}\left(Y^{+}\right) \mapsto \mathbb{N} \backslash\{0\}$ be the generalized multiplicity of $Y^{+}$.

Let $f_{l}^{-}, z_{j}^{-}, \Sigma_{j}^{-}, g_{k}^{-}, \theta_{\Gamma}\left(z_{j}^{-}\right), Y^{-}, \eta_{Y^{-}}$be the analog for the interval $[-a, 0]$ of the spaces, functions, points, families and densities that we used in the construction on the interval $[0, a]$.

Since $\theta_{\Lambda_{i-1}^{N}}(p)=\sum_{j=1}^{h^{ \pm}} \theta_{\Lambda_{i-1}^{N}}\left(z_{j}^{ \pm}\right)$and, by construction, $\theta_{\Lambda_{i-1}^{N}}\left(z_{j}^{ \pm}\right)=\eta_{Y^{ \pm}}\left(z_{j}^{ \pm}\right)$we can apply Lemma 4.9 and find a system of curves in $H^{2, p}(\mathbb{S})$, which will be our $\Lambda_{i}^{N}$, whose trace and density function outside $R\left(p_{i}\right)$ are the same as $\Lambda_{i-1}^{N}$, while on $R^{+}\left(p_{i}\right)$ (respectively on $R^{-}\left(p_{i}\right)$ ) the trace is given by $\operatorname{graph}\left(Y^{+}\right)\left(\operatorname{respectively} \operatorname{graph}\left(Y^{-}\right)\right.$) and the density function agrees with $\eta_{Y^{+}}$(respectively with $\eta_{Y^{-}}$). By construction, and recalling Remark 4.6, properties (i), (ii) and (iv) hold (note that $\Lambda_{i}^{N}$ verifies the finiteness property over $\bigcup_{j \leqslant i} R\left(p_{j}\right)$ ).

To prove the validity of (iii) we need the concept of canonical family. Since the supports of the system of curves coincide outside $R\left(p_{i}\right)$ it is enough to verify inequality (iii) inside $R\left(p_{i}\right)$.

Using Lemma 4.11 we can choose a canonical family $\left\{\left(f_{1}^{+}, \mu_{1}^{+}\right), \ldots,\left(f_{r^{+}}^{+}, \mu_{r^{+}}^{+}\right)\right\}$for $\left(\Lambda_{i-1}^{N}\right)$ in $R^{+}\left(p_{i}\right)$. Observe that $\left\{z_{1}^{+}, \ldots, z_{h}^{+}\right\}=\left\{\left(a, f_{1}^{+}(a)\right), \ldots,\left(a, f_{r}^{+}(a)\right)\right\}$ and $h \leqslant r^{+} \leqslant \theta_{\Lambda_{i-1}^{N}}\left(p_{i}\right)$.

Recalling Definition 4.10 it follows

$$
\mathcal{F}\left(\Lambda_{i-1}^{N},\left(\Lambda_{i-1}^{N}\right)^{-1}\left(R\left(p_{i}\right)\right)\right)=\sum_{l=1}^{r^{+}} \mu_{l}^{+} \mathcal{P}\left(f_{l}^{+},\right] 0, a[)+\sum_{l=1}^{r^{-}} \mu_{l}^{-} \mathcal{P}\left(f_{l}^{-},\right]-a, 0[) .
$$

Note also that

$$
\sum_{l: f_{l}^{ \pm}(a)=z_{j}^{ \pm}} \mu_{l}^{ \pm}=\theta_{\Lambda_{i-1}^{N}}\left(z_{j}^{ \pm}\right) \quad \forall j \in\{1, \ldots, h\} .
$$


We now group the terms in the summation $\sum_{l=1}^{r^{+}}$as follows:

$$
\sum_{l=1}^{r^{+}} \mu_{l}^{+} \mathcal{P}\left(f_{l}^{+},\right] 0, a[)=\sum_{j=1}^{h^{+}} \sum_{l: f_{l}^{+}(a)=z_{j}^{+}} \mu_{l}^{+} \mathcal{P}\left(f_{l}^{+},\right] 0, a[) .
$$

We observe that for any $j$ and any $l$ such that $f_{l}^{+}(a)=z_{j}^{+}$, the minimality property of $g_{j}^{+}$entails

$$
\mathcal{P}\left(f_{l}^{+},\right] 0, a[) \geqslant \mathcal{P}\left(g_{j}^{+},\right] 0, a[) .
$$

Therefore, from (32)-(34) we deduce

$$
\sum_{l=1}^{r^{+}} \mu_{l}^{+} \mathcal{P}\left(f_{l}^{+},\right] 0, a[) \geqslant \sum_{j=1}^{h^{+}} \theta_{\Lambda_{i-1}^{N}}\left(z_{j}^{+}\right) \mathcal{P}\left(g_{j}^{+},\right] 0, a[) .
$$

Similarly

$$
\sum_{l=1}^{r^{-}} \mu_{l}^{-} \mathcal{P}\left(f_{l}^{-},\right]-a, 0[) \geqslant \sum_{j=1}^{h^{-}} \theta_{\Lambda_{i-1}^{N}}\left(z_{j}^{-}\right) \mathcal{P}\left(g_{j}^{-},\right]-a, 0[) .
$$

Using (31), (35) and (36) it follows

$$
\begin{aligned}
\mathcal{F}\left(\Lambda_{i-1}^{N},\left(\Lambda_{i-1}^{N}\right)^{-1}\left(R\left(p_{i}\right)\right)\right) & \geqslant \sum_{j=1}^{h^{+}} \theta_{\Lambda_{i-1}^{N}}\left(z_{j}^{+}\right) \mathcal{P}\left(g_{j}^{+},\right] 0, a[)+\sum_{j=1}^{h^{-}} \theta_{\Lambda_{i-1}^{N}}\left(z_{j}^{-}\right) \mathcal{P}\left(g_{j}^{-},\right]-a, 0[) \\
& =\mathcal{F}\left(\Lambda_{i}^{N},\left(\Lambda_{i}^{N}\right)^{-1}\left(R\left(p_{i}\right)\right)\right),
\end{aligned}
$$

and (iii) follows.

We now define

$$
\Lambda_{N}:=\Lambda_{m(N)}^{N} .
$$

We have

$$
\left(\Lambda_{N}\right)=\left(\Lambda_{m(N)}^{N}\right) \subseteq\left(\Lambda_{m(N)-1}^{N}\right) \subseteq \cdots \subseteq\left(\Lambda_{0}^{N}\right)=(\Gamma) .
$$

Consequently $\operatorname{Sing}_{\Lambda_{N}} \subseteq \operatorname{Sing}_{\Gamma}$, and by construction $\operatorname{Sing}_{\Lambda_{N}}=\operatorname{Nod}_{\Lambda_{N}}$. Furthermore, since $\Lambda_{N}$ verifies the finiteness property on $\bigcup_{i=1}^{m(N)} R\left(p_{i}\right) \supseteq \operatorname{Sing}_{\Gamma}$ and $\left(\Lambda_{N}\right) \subseteq(\Gamma)$, we have that $\left(\Lambda_{N}\right)$ verifies the finiteness property on $\mathbb{R}^{2}$. Finally, from (37), we have

$$
\mathcal{F}\left(\Lambda_{N}\right)=\mathcal{F}\left(\Lambda_{m(N)}^{N}\right) \leqslant \mathcal{F}\left(\Lambda_{m(N)-1}^{N}\right) \leqslant \cdots \leqslant \mathcal{F}(\Gamma),
$$

and this concludes the proof of step 1.

Step 2 . We prove that $\left\{\Lambda_{N}\right\}$ has a subsequence weakly converging in $H^{2, p}(\mathbb{S})$ to a system of curves $\Lambda$, which is not necessarily a limit system of curves, but is equivalent to $\Gamma$.

Since $\left(\Lambda_{N}\right) \subseteq(\Gamma)$ and

$$
\sup _{N \in \mathbb{N}} \mathcal{F}\left(\Lambda_{N}\right) \leqslant \mathcal{F}(\Gamma)
$$

we can apply Theorem 3.1 of [4] and find a subsequence (still indicated by $\left\{\Lambda_{N}\right\}$ ) which converges weakly in $H^{2, p}(\mathbb{S})$ as $N \rightarrow+\infty$ to a system of curves $\Lambda$ such that $(\Lambda) \subseteq(\Gamma)$.

We want to prove that $\Lambda \sim \Gamma$. To this aim we want to use Lemma 3.11. We start by proving that $(\Lambda)=(\Gamma)$. Let $p \in \operatorname{Reg}_{\Gamma}$. Since $\operatorname{Sing}_{\Gamma}=(\Gamma) \backslash \operatorname{Reg}_{\Gamma}$ is compact, we have $\operatorname{dist}\left(p, \operatorname{Sing}_{\Gamma}\right)>0$. So, for every $N$ with $1 / 2^{N}<\operatorname{dist}\left(p, \operatorname{Sing}_{\Gamma}\right)$, the point $p$ is outside the region where we made our modifications and therefore there 
is a whole neighborhood of $p$ where the support of $\Lambda_{N}$ and its density function are the same as the support and the density of $\Gamma$. Therefore $p \in \operatorname{Reg}_{\Lambda}$ and $\operatorname{Reg}_{\Gamma} \subseteq \operatorname{Reg}_{\Lambda}$. Hence, recalling (13) and the inclusion $(\Lambda) \subseteq(\Gamma)$, we get $(\Gamma)=\overline{\operatorname{Reg}_{\Gamma}} \subseteq(\Lambda)$. So $(\Gamma)=(\Lambda)$ and therefore $\operatorname{Reg}_{\Gamma}=\operatorname{Reg}_{\Lambda}$.

By construction we have $\theta_{\Gamma}=\theta_{\Lambda}$ on $\operatorname{Reg}_{\Gamma}=\operatorname{Reg}_{\Lambda}$. Hence $\Gamma \sim \Lambda$ by Lemma 3.11.

Step 3. Construction of the sequence $\left\{\widetilde{\Gamma}_{N}\right\}$.

Let us fix $N \in \mathbb{N}$. As $\Lambda_{N}$ verifies the finiteness property we can apply Theorem 2.24 and find a parameter space $\widetilde{\mathbb{S}}_{N}$ and a limit system of curves $\widetilde{\Gamma}_{N} \in H^{2, p}\left(\widetilde{\mathbb{S}}_{N}\right)$ such that: $\widetilde{\mathbb{S}}_{N}$ has a number of connected components uniformly bounded with respect to $N ; \widetilde{\Gamma}_{N} \sim \Lambda_{N}$ and $\widetilde{\Gamma}_{N}$ is the strong $H^{2, p}$-limit of a sequence $\left\{\Gamma_{N, h}\right\}_{h} \subset H^{2, p}\left(\widetilde{\mathbb{S}}_{N}\right)$ of oriented parametrizations of bounded smooth open sets with equibounded energy and $L^{1}\left(\mathbb{R}^{2}\right)$-converging to $A_{\widetilde{\Gamma}_{N}}=A_{\Lambda_{N}}^{o}$. Passing to a suitable subsequence (still labelled by the index $N$ ) we can suppose that $\widetilde{\mathbb{S}}_{N}=\widetilde{\mathbb{S}}_{\text {for }}$ any $N$.

Since $\widetilde{\Gamma}_{N}$ and $\Lambda_{N}$ are equivalent, from step 1 we have $\operatorname{Sing}_{\widetilde{\Gamma}_{N}}=\operatorname{Nod}_{\widetilde{\Gamma}_{N}} \subseteq \operatorname{Sing}_{\Gamma}$ and, using Lemma 3.9, we have

$$
\mathcal{F}\left(\widetilde{\Gamma}_{N}\right)=\mathcal{F}\left(\Lambda_{N}\right) \leqslant \mathcal{F}(\Gamma) .
$$

Furthermore from step 1 we also have $\left(\widetilde{\Gamma}_{N}\right)=\left(\Lambda_{N}\right) \subseteq(\Gamma)$. Therefore we can apply Theorem 2.10 and find a subsequence (still indicated by $\widetilde{\Gamma}_{N}$ ) whose elements are all defined on $\widetilde{\mathbb{S}}$, and weakly converging in $H^{2, p}$ to a system of curves $\widetilde{\Gamma} \in H^{2, p}(\widetilde{\mathbb{S}})$. Using the same arguments of step 2 , one can prove that $\widetilde{\Gamma} \sim \Gamma$.

Finally

$$
\mathcal{F}(\Gamma)=\mathcal{F}(\widetilde{\Gamma}) \leqslant \liminf _{N \rightarrow \infty} \mathcal{F}\left(\widetilde{\Gamma}_{N}\right) \leqslant \limsup _{N \rightarrow \infty} \mathcal{F}\left(\widetilde{\Gamma}_{N}\right) \leqslant \mathcal{F}(\Gamma),
$$

and therefore $\mathcal{F}(\Gamma)=\lim _{N \rightarrow \infty} \mathcal{F}\left(\widetilde{\Gamma}_{N}\right)$.

Fig. 7 illustrates the construction of the sequence $\left\{\widetilde{\Gamma}_{N}\right\}$ of Theorem 5.1 in a particular situation.

The following result is an improvement of Theorem 2.24 .

Corollary 5.2. Let $\Gamma$ be a system of curves of class $H^{2, p}(\mathbb{S})$ without crossings. Then there exist a parameter space $\widetilde{\mathbb{S}}$, a system of curves $\widetilde{\Gamma} \in H^{2, p}(\widetilde{\mathbb{S}}) \cap \mathcal{A}\left(A_{\Gamma}^{o}\right)$ equivalent to $\Gamma$ and a sequence $\left\{\Gamma_{N}\right\}$ of oriented parametrizations of bounded open smooth sets $E_{N} \subset \mathbb{R}^{2}$, such that

$$
E_{N} \rightarrow A_{\Gamma}^{o} \quad \text { in } L^{1}\left(\mathbb{R}^{2}\right), \quad \Gamma_{N} \rightarrow \widetilde{\Gamma} \quad \text { weakly in } H^{2, p}(\widetilde{\mathbb{S}}), \quad \lim _{N \rightarrow \infty} \mathcal{F}\left(\Gamma_{N}\right)=\mathcal{F}(\Gamma) .
$$

In particular

$$
\overline{\mathcal{F}}\left(A_{\Gamma}^{o}\right)<+\infty .
$$

Proof. Let $\widetilde{\Gamma} \in H^{2, p}(\mathbb{S})$ and $\left\{\widetilde{\Gamma}_{N}\right\}$ be as in Theorem 5.1. The convergence of the energies, together with the weak convergence, implies that $\lim _{N \rightarrow \infty}\left\|\widetilde{\Gamma}_{N}\right\|_{2, p}=\|\widetilde{\Gamma}\|_{2, p}$, hence the strong $H^{2, p}$-convergence of $\left\{\widetilde{\Gamma}_{N}\right\}$ to $\widetilde{\Gamma}$. Write $\Gamma_{N, h}:=\partial E_{N, h}$, where $\Gamma_{N, h}$ are introduced in the proof of step 3 in Theorem 5.1. Using a diagonal argument we can select a subsequence $\left\{E_{N, h_{N}}\right\}$, which for simplicity we denote by $\left\{E_{N}\right\}$, such that the sequence $\left\{\Gamma_{N}\right\}$ of the oriented parametrizations of the elements of $\left\{E_{N}\right\}$ converges strongly in $H^{2, p}(\widetilde{\mathbb{S}})$ to $\widetilde{\Gamma}$. Therefore, since $\widetilde{\Gamma} \sim \Gamma$, we have

$$
\lim _{N \rightarrow \infty} \mathcal{F}\left(\Gamma_{N}\right)=\mathcal{F}(\widetilde{\Gamma})=\mathcal{F}(\Gamma) .
$$

It remains to prove that $E_{N} \rightarrow E$ in $L^{1}\left(\underset{\mathbb{R}^{2}}{\tilde{\Gamma}}\right)$ and that $\widetilde{\Gamma} \in \mathcal{A}(E)$. For every $N \in \mathbb{N}$ we have $\chi_{E_{N}}(z)=\mathcal{I}\left(\Gamma_{N}, z\right)$ for every $z \in \mathbb{R}^{2} \backslash\left(\Gamma_{N}\right)$ and $\chi_{A_{\widetilde{\Gamma}}}(z)=\mathcal{I}(\widetilde{\Gamma}, z)$ for every $z \in \mathbb{R}^{2} \backslash(\widetilde{\Gamma})$. By the continuity property of the index and the Dominated Convergence Theorem we have that $E_{N}=A_{\Gamma_{N}} \rightarrow A_{\widetilde{\Gamma}}$ in $L^{1}\left(\mathbb{R}^{2}\right)$ as $N \rightarrow \infty$. Using the fact that $\widetilde{\Gamma} \sim \Gamma$ we have $\mathcal{A}_{\widetilde{\Gamma}}=\mathcal{A}_{\Gamma}^{o}=E$, so that $E_{N} \rightarrow E$ in $L^{1}\left(\mathbb{R}^{2}\right)$. Moreover, $\widetilde{\Gamma} \in \mathcal{A}(E)$. 


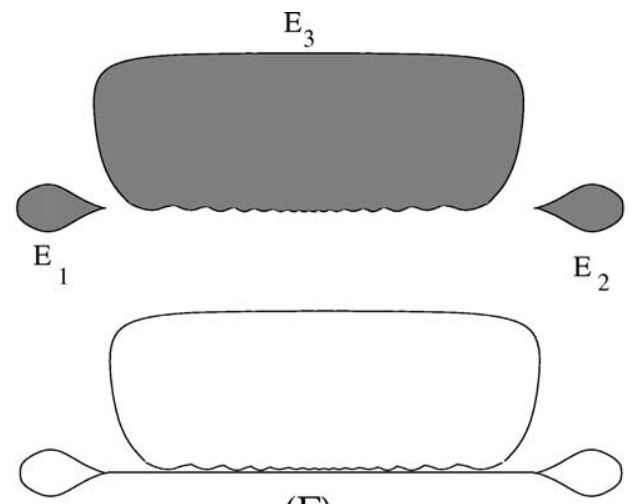

$(\Gamma)$

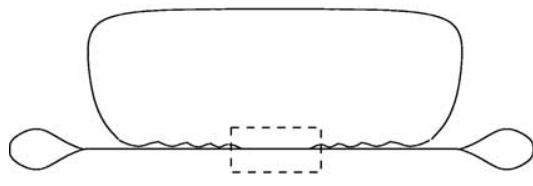

$\left(\tilde{\Gamma}_{\mathrm{N}}\right)$

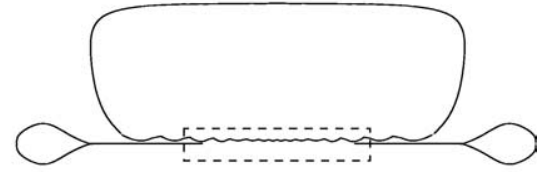

$\left(\Gamma_{\mathrm{n}}\right)$

Fig. 7. The set $E:=E_{1} \cup E_{2} \cup E_{3}$ has smooth boundary except for the simple cusps of $E_{1}$ and $E_{2}$. The boundary of the smooth connected component $E_{3}$ oscillates and meets (from above) infinitely many times the horizontal line connecting the two cusps. Let $\Gamma \in \mathcal{A}(E)$ be such that $\theta_{\Gamma}=1$ on $\operatorname{Reg}_{\Gamma} \cap \partial E$ and $\theta_{\Gamma}=2$ on $\operatorname{Reg}_{\Gamma} \cap\left(\mathbb{R}^{2} \backslash \partial E\right)$. The system $\widetilde{\Gamma}_{N}$ is obtained through the desingularization procedure described in Theorem 5.1, while the system $\Gamma_{n}$ is obtained through the desingularization procedure of Theorem 6.3. The main difference between the two systems is explained in Remark 6.5. These two systems of curves are equivalent to $\Gamma$ out of the two respective (dotted) nice rectangles, and have density constantly equal to 3 inside the rectangles. The energies of the systems $\Gamma_{n}$ converge to $\overline{\mathcal{F}}(E)$ (this will be a consequence of the results of Section 8) whereas the sequence itself does not converge to an element of $\mathcal{Q}_{\text {fin }}(E)$.

Remark 5.3. Inequality (41) was proved in [4, Theorem 6.2], under the further assumption that $\Gamma$ satisfies the finiteness property. Removing this assumption is one of the interesting and useful aspects of Corollary 5.2 (and of Theorem 5.1).

\section{Representation formulas for $\overline{\mathcal{F}}$}

According to Theorem 2.25, the functional $\overline{\mathcal{F}}(E, \cdot)$ is not local. As a consequence, $\overline{\mathcal{F}}$ does not admit an integral representation. In this section we study how to represent $\overline{\mathcal{F}}$ as a minimum problem involving $\mathcal{F}$, considered as a functional defined on systems of curves. Using tools of geometric measure theory (namely generalized Gauss graphs) in [10] there are some partial results in this direction.

The following result is an improvement of (9).

Proposition 6.1. Let $E \subset \mathbb{R}^{2}$ be such that $\overline{\mathcal{F}}(E)<+\infty$. Then

$$
\overline{\mathcal{F}}(E)=\min \{\mathcal{F}(\Gamma): \Gamma \in \mathcal{A}(E)\}=\min \left\{\mathcal{F}(\Gamma): \Gamma \in \mathcal{A}^{o}(E)\right\} .
$$

Proof. Thanks to (9), to show the first equality in (42) it is enough to prove that

$$
\overline{\mathcal{F}}(E) \leqslant \inf \{\mathcal{F}(\Gamma): \Gamma \in \mathcal{A}(E)\}
$$


and that the infimum in (43) is attained. Given $\Gamma \in \mathcal{A}(E)$, let $\left\{\Gamma_{N}\right\}$ and $\left\{E_{N}\right\}$ be as in Corollary 5.2. Recalling that $A_{\Gamma}=A_{\Gamma}^{o},\left|E \Delta A_{\Gamma}\right|=0$, using (5) and (40) we have

$$
\overline{\mathcal{F}}(E) \leqslant \liminf _{h \rightarrow+\infty} \mathcal{F}\left(E_{N}\right)=\lim _{N \rightarrow+\infty} \mathcal{F}\left(\Gamma_{N}\right)=\mathcal{F}(\Gamma),
$$

and (43) follows.

Now we select a sequence $\left\{E_{h}\right\}$ of smooth bounded open sets converging to $E$ in $L^{1}\left(\mathbb{R}^{2}\right)$ and such that $\lim _{h \rightarrow+\infty} \mathcal{F}\left(E_{h}\right)=\overline{\mathcal{F}}(E)$. As in the proof of [4, Lemma 3.3], we can find a parameter space $\mathbb{S}$, a system $\Gamma \in H^{2, p}(\mathbb{S})$ and a sequence $\left\{\Gamma_{h}\right\} \subset H^{2, p}(\mathbb{S})$ of oriented parametrizations of smooth bounded open sets $E_{h}^{\prime} \subseteq E_{h}$, $\partial E_{h}^{\prime} \subseteq \partial E_{h}$, such that $\left(\Gamma_{h}\right)$ are all contained in a bounded subset of $\mathbb{R}^{2}$ independent of $h, E_{h}^{\prime} \rightarrow E$ in $L^{1}\left(\mathbb{R}^{2}\right)$ and $\Gamma_{h} \rightarrow \Gamma$ weakly in $H^{2, p}(\mathbb{S})$ as $h \rightarrow+\infty$. To show that the infimum in (43) is attained, it is enough to observe that $\Gamma \in \mathcal{A}(E)$ and

$$
\overline{\mathcal{F}}(E)=\lim _{h \rightarrow+\infty} \mathcal{F}\left(E_{h}\right) \geqslant \liminf _{h \rightarrow+\infty} \mathcal{F}\left(E_{h}^{\prime}\right)=\liminf _{h \rightarrow+\infty} \mathcal{F}\left(\Gamma_{h}\right) \geqslant \mathcal{F}(\Gamma) \geqslant \overline{\mathcal{F}}(E),
$$

where we used the weak $H^{2, p}$ lower semicontinuity of $\mathcal{F}$ on systems of curves and (43).

Let us now prove that $\overline{\mathcal{F}}(E)=\min \left\{\mathcal{F}(\Gamma): \Gamma \in \mathcal{A}^{o}(E)\right\}$. As a direct consequence of the above arguments and the inclusion $\mathcal{A}(E) \subseteq \mathcal{A}^{o}(E)$ we have $\overline{\mathcal{F}}(E) \geqslant \inf \left\{\mathcal{F}(\Gamma): \Gamma \in \mathcal{A}^{o}(E)\right\}$. On the other hand, the opposite inequality can be proved as in the proof of (43), using the fact that $\left|E \Delta A_{\Gamma}^{o}\right|=0$. Eventually, the proof that the infimum in $\mathcal{A}^{o}(E)$ is attained follows from the inclusion $A^{o}(E) \supseteq A(E)$ and the above observations.

Definition 6.2. Let $E \subset \mathbb{R}^{2}$ be such that $\overline{\mathcal{F}}(E)<+\infty$. Any $\Gamma \in \mathcal{A}(E)$ (respectively $\Gamma \in \mathcal{A}^{o}(E)$ ) satisfying $\mathcal{F}(\Gamma)=\overline{\mathcal{F}}(E)$ will be called a minimal system of curves in $\mathcal{A}(E)$ (respectively in $\mathcal{A}^{o}(E)$ ).

Theorem 6.3. Let $E \subset \mathbb{R}^{2}$ be such that $\overline{\mathcal{F}}(E)<+\infty$ and suppose that $\operatorname{Sing}_{\partial E^{*}}$ is a finite set. Let $\Gamma \in \mathcal{A}(E)$. Then there exist a sequence $\left\{\Gamma_{n}\right\} \subset \mathcal{Q}_{\text {fin }}(E)$ and a system of curves $\widetilde{\Gamma} \sim \Gamma$ such that

$$
\Gamma_{n} \rightarrow \widetilde{\Gamma} \quad \text { weakly in } H^{2, p}, \quad \lim _{n \rightarrow+\infty} \mathcal{F}\left(\Gamma_{n}\right)=\mathcal{F}(\Gamma) .
$$

In particular

$$
\overline{\mathcal{F}}(E)=\inf \left\{\mathcal{F}(\Gamma): \Gamma \in \mathcal{Q}_{\text {fin }}(E)\right\} .
$$

Remark 6.4. The set $\mathcal{Q}_{\text {fin }}(E)$ is not empty only if $\partial E^{*}$ has a finite number of singularities. Indeed, for every $\Gamma \in \mathcal{A}(E)$ we have $\operatorname{Sing}_{\Gamma} \supseteq \operatorname{Sing}_{\partial E^{*}}$; therefore, if $\operatorname{Sing}_{\partial E^{*}}$ is infinite, $\Gamma$ cannot verify the finiteness property.

Remark 6.5. The main difference between Theorem 6.3 and Theorem 5.1 is that in Theorem 6.3 we are able to approximate $\Gamma$ under the additional constraint that

$$
E^{*}=\operatorname{int}\left(A_{\Gamma_{n}} \cup\left(\Gamma_{n}\right)\right) \quad \forall n \in \mathbb{N} .
$$

The difficulty to keep (45) true is related to the following observation: even if the singular points of $\partial E^{*}$ are isolated, it may happen that they are accumulation points of singularities of $(\Gamma)$, see Fig. 8; similarly, there may be (an infinite number of) regular points of $\partial E^{*}$ which are singular points (or accumulation points of singular points) of $(\Gamma)$.

Remark 6.6. We shall see in Section 8.1 that the infimum in (44) in general is not achieved. 


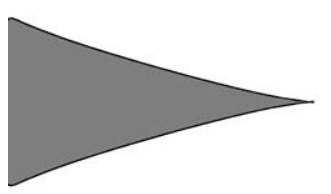

$\mathrm{E}$

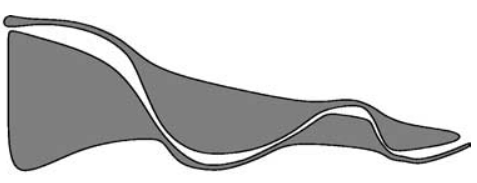

$\mathrm{E}_{\mathrm{n}}$

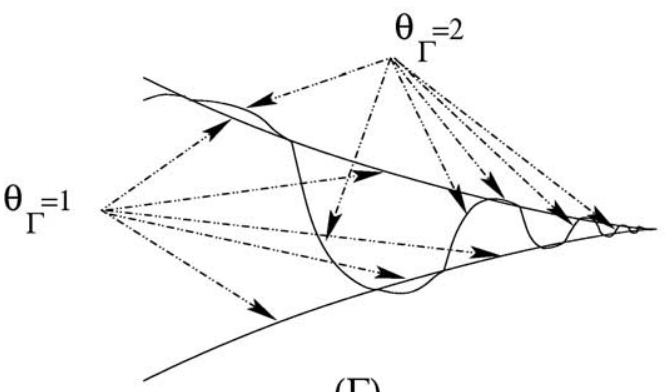

$(\Gamma)$

Fig. 8. A cusp of $\partial E$ which is accumulation point of singular points of $\Gamma$.

Proof of Theorem 6.3. Write $\Gamma=\left\{\gamma_{1}, \ldots, \gamma_{m}\right\}: \mathbb{S} \rightarrow \mathbb{R}^{2}$. Recall that, as $\partial E^{*} \subseteq(\Gamma)$, we have $\operatorname{Sing}_{\partial E^{*}} \subseteq \operatorname{Sing}_{\Gamma}$. Let $\operatorname{acc}_{\operatorname{sing}}(\Gamma)$ be the set of the accumulation points of $\operatorname{Sing}_{\Gamma}$. Fix $n \in \mathbb{N}$. For every $p \in \operatorname{acc}_{\operatorname{sing}}(\Gamma)$ let $R(p)$ be a nice rectangle for $(\Gamma)$ at $p$ with diameter less than $2^{-n}$ such that:

- $(\Gamma) \cap \partial R(p) \subset \operatorname{Reg}_{\Gamma}$ (recall Corollary 3.4);

- if $p \notin \partial E^{*}$ then $R(p) \Subset \mathbb{R}^{2} \backslash \partial E^{*}$;

- if $p \in \operatorname{Sing}_{\partial E^{*}}$ then $\partial E^{*}$ is represented in $R^{+}(p)$ (or in $R^{-}(p)$ ) by a finite union of graphs of $H^{2, p}$ functions, all passing through $p$, that do not intersect each other at any point of $R^{+}(p) \backslash\{p\}$ (or of $R^{-}(p) \backslash\{p\}$ ) and

$$
\mathcal{F}\left(\Gamma, \Gamma^{-1}(R(p))\right)<\frac{1}{M C 2^{n}}, \quad C:=\sharp \operatorname{Sing}_{\partial E^{*}}, \quad M:=\left\|\theta_{\Gamma}\right\|_{L^{\infty}\left((\Gamma), \mathcal{H}^{1}\right)} .
$$

Note that these graphs coincide with all points of $(\Gamma) \cap(R(p) \backslash\{p\}$ ) with odd density (in general $p$ may have even density, for example if it is a cusp point of $\left.\partial E^{*}\right)$.

Select a finite family $\left\{R\left(p_{1}\right), \ldots, R\left(p_{\delta(n)}\right)\right\}$ covering the set $\operatorname{acc}_{\operatorname{sing}}(\Gamma)$. Since $\operatorname{Sing}_{\partial E^{*}}$ is finite, we can also assume that $\left\{R\left(p_{1}\right), \ldots, R\left(p_{\delta(n)}\right)\right\}$ satisfies the following additional property:

$$
p \in \operatorname{Sing}_{\partial E^{*}} \cap \operatorname{acc} \operatorname{sing}(\Gamma) \Rightarrow R(p) \in\left\{R\left(p_{1}\right), \ldots, R\left(p_{\delta(n)}\right)\right\} .
$$

The construction of $\left\{\Gamma_{n}\right\}$ is divided into two steps.

Step 1 . We construct a sequence $\left\{\Lambda_{n}\right\} \subset H^{2, p}(\mathbb{S})$ of systems of curves such that

(a) $\partial E^{*} \subseteq\left(\Lambda_{n}\right) \subseteq(\Gamma)$;

(b) $\left|A_{\Lambda_{n}}^{o} \Delta E^{*}\right|=0$;

(c) Sing $\Lambda_{\Lambda_{n}} \cap \partial E^{*}$ is a finite set;

(d) $\lim _{n \rightarrow+\infty} \mathcal{F}\left(\Lambda_{n}\right)=\mathcal{F}(\Gamma)$.

In the construction of $\left\{\Lambda_{n}\right\}$ we are not able to bound the energy of $\Lambda_{n}$ with the energy of $\Gamma$; however, we can prove that condition (d) is valid, and at the same time the constraint in condition (b) is fulfilled. 
In order to construct $\Lambda_{n}$ we use a recursive algorithm consisting of $\delta(n)$ steps. Let $\Lambda_{0}^{n}:=\Gamma$, let $1 \leqslant i \leqslant \delta(n)$ and suppose that the system $\Lambda_{i-1}^{n}$ of curves of class $H^{2, p}$ has been defined. Then $\Lambda_{i}^{n}$ is obtained by modifying $\Lambda_{i-1}^{n}$ only on $\operatorname{int}\left(R\left(p_{i}\right)\right)$, in such a way that:

(i) the set of the points of $\left(\Lambda_{i}^{n}\right)$ where $\theta_{\Lambda_{i}^{n}}$ is odd is the same as the set of the points of $\left(\Lambda_{i-1}^{n}\right)$ where $\theta_{\Lambda_{i-1}^{n}}$ is odd;

(ii) $\operatorname{Sing}_{\Lambda_{i}^{n}} \cap R\left(p_{i}\right) \cap \partial E^{*}$ is a finite set;

(iii) the following estimate holds:

$$
\left|\mathcal{F}\left(\Lambda_{i-1}^{n},\left(\Lambda_{i-1}^{n}\right)^{-1}\left(R\left(p_{i}\right)\right)\right)-\mathcal{F}\left(\Lambda_{i}^{n},\left(\Lambda_{i}^{n}\right)^{-1}\left(R\left(p_{i}\right)\right)\right)\right| \leqslant \begin{cases}\frac{1}{C 2^{n}} & \text { if } p_{i} \in \operatorname{Sing}_{\partial E^{*}}, \\ \frac{M}{\delta(n) 2^{n}} & \text { if } p_{i} \in \operatorname{Reg}_{\partial E^{*}} .\end{cases}
$$

We suppose $p_{i}=0$ and $R\left(p_{i}\right)=[-a, a] \times[-b, b]$. If either $p_{i} \in \mathbb{R}^{2} \backslash \partial E^{*}$ or $p_{i} \notin \operatorname{acc} \operatorname{sing}_{\text {sing }}\left(\Lambda_{i-1}^{n}\right)$ then we set $\Lambda_{i}^{n}:=\Lambda_{i-1}^{n}$ in $R\left(p_{i}\right)$, and (i)-(iii) are trivially satisfied.

Let us now suppose that $p_{i} \in \operatorname{acc} \operatorname{sing}\left(\Lambda_{i-1}^{n}\right) \cap \partial E^{*}$. Write

$$
R^{+}\left(p_{i}\right) \cap \partial E^{*}=\bigcup_{l=1}^{k} \operatorname{graph}\left(\phi_{l}^{+}\right),
$$

with $k \geqslant 1, \phi_{l}^{+} \in H^{2, p}(] 0, a[), \phi_{l}^{+}(0)=0$ for every $l=1, \ldots, k$ and $\phi_{l}^{+}<\phi_{j}^{+}$on $\left.] 0, a\right]$ for $1 \leqslant l<j \leqslant k(k=1$ if $p_{i} \in \operatorname{Reg}_{\partial E^{*}}$ ).

Define

$$
\Psi:\left(\Lambda_{i-1}^{n}\right) \cap R^{+}\left(p_{i}\right) \rightarrow 2 \mathbb{N}, \quad \Psi:= \begin{cases}\theta_{\Lambda_{i-1}^{n}}-1 & \text { on } \bigcup_{l=1}^{k} \operatorname{graph}\left(\phi_{l}^{+}\right), \\ \theta_{\Lambda_{i-1}^{n}} & \text { otherwise in }\left(\Lambda_{i-1}^{n}\right) \cap R^{+}\left(p_{i}\right),\end{cases}
$$

and set $X:=\left\{q \in \operatorname{int}\left(R^{+}\left(p_{i}\right)\right): \Psi(q)>0\right\}$. As $p_{i} \in \operatorname{acc} \operatorname{sing}\left(\Lambda_{i-1}^{n}\right)$, from (16) it follows that $\theta_{\Lambda_{i-1}^{n}}\left(p_{i}\right)>1$, hence $p_{i} \in X$. Since $\theta_{\Lambda_{i-1}^{n}}$ verifies the train tracks property in $\operatorname{int}\left(R^{+}\left(p_{i}\right)\right)$ and $\left(\Lambda_{i-1}^{n}\right) \cap \operatorname{int}\left(R^{+}\left(p_{i}\right)\right)$ is a finite union of $H^{2, p}$ graphs, we have that also $X$ is a finite union of $H^{2, p}$ graphs meeting tangentially and passing through zero horizontally. Furthermore $\Psi_{\mid X}$ verifies the train tracks property and is upper semicontinuous in $\operatorname{int}\left(R^{+}\left(p_{i}\right)\right)$. Finally, we remark that, since the set of points where $\theta_{\Lambda_{i-1}^{n}}$ is odd coincides with $\cup_{l=1}^{k} \operatorname{graph}\left(\phi_{l}^{+}\right)$(possibly, for $k>1$, with the exclusion of $p_{i}$ ), then $\Psi_{\left.\right|_{X}}$ is everywhere even.

Arguing as in the proof of Lemma 4.11 we construct a canonical family

$$
Y^{+}:=\left\{\left(f_{1}^{+}, 2 \mu_{1}^{+}\right), \ldots,\left(f_{r^{+}}^{+}, 2 \mu_{r^{+}}^{+}\right)\right\} \subset H^{2, p}(] 0, a[) \times(2 \mathbb{N} \backslash\{0\})
$$

for $(\Psi, X)$ in $R^{+}\left(p_{i}\right)$, hence

$$
X=\operatorname{graph}\left(Y^{+}\right), \quad \Psi=\eta_{Y^{+}} \quad \text { on } X .
$$

We now define

$$
\widehat{Y}^{+}:=\left\{\left(\phi_{1}^{+}, 1\right), \ldots,\left(\phi_{k}^{+}, 1\right),\left(f_{1}^{+}, 2 \mu_{1}^{+}\right), \ldots,\left(f_{r^{+}}^{+}, 2 \mu_{r^{+}}^{+}\right)\right\} .
$$

We have

$$
\operatorname{graph}\left(\widehat{Y}^{+}\right)=\left(\Lambda_{i-1}^{n}\right) \cap R^{+}\left(p_{i}\right), \quad \eta_{\widehat{Y}^{+}}=\theta_{\Lambda_{i-1}^{n}} \quad \text { on } \operatorname{graph}\left(\widehat{Y}^{+}\right) .
$$

If $R^{-}\left(p_{i}\right) \cap \partial E^{*} \neq \emptyset$ we repeat the same construction in $R^{-}\left(p_{i}\right)$. We now proceed in two different ways depending on whether $p_{i} \in \operatorname{Sing}_{\partial E^{*}}$ or $p_{i} \in \operatorname{Reg}_{\partial E^{*}}$. The case $p_{i} \in \operatorname{Sing}_{\partial E^{*}}$ is easier, since by assumption $\operatorname{Sing}_{\partial E^{*}}$ is finite. On the other hand, there may be an infinite number of regular points of $\partial E^{*}$ which are accumulation points of singular points of $\Gamma$, and this makes case 2 more delicate. 
Case 1 of step 1 . Suppose $p_{i} \in \operatorname{acc}_{\operatorname{sing}}\left(\Lambda_{i-1}^{n}\right) \cap \operatorname{Sing}_{\partial E^{*}}$ (a situation like the one depicted in Fig. 8).

In this case we have $k>1$. Let $l \in\left\{1, \ldots, r^{+}\right\}$and define

$$
\xi_{l}:=\sup \left\{x \in[0, a]:\left(x, f_{l}^{+}(x)\right) \in \bigcup_{j=1}^{k} \operatorname{graph}\left(\phi_{j}^{+}\right)\right\} .
$$

We replace the function $f_{l}^{+}$with the function $g_{l}^{+}$defined as follows: if $\xi_{l}=0$ then $g_{l}^{+}:=f_{l}^{+}$. If $\left.\left.\xi_{l} \in\right] 0, a\right]$ there is a unique $j \in\{1, \ldots, k\}$ such that $f_{l}^{+}\left(\xi_{l}\right)=\phi_{j}^{+}\left(\xi_{l}\right)$; in this case we set

$$
g_{l}^{+}:= \begin{cases}\phi_{j}^{+} & \text {on }\left[0, \xi_{l}\right] \\ f_{l}^{+} & \text {on }\left[\xi_{l}, a\right]\end{cases}
$$

Roughly speaking, the above definition means that the graph of $g_{l}^{+}$coincides with $\partial E^{*}$ in a small halfneighborhood of $p_{i}$, thus leading by construction to the finiteness property of $\Lambda_{i}^{n}$ on $R^{+}\left(p_{i}\right) \cap \partial E^{*}$.

Define

$$
Z^{+}:=\left\{\left(\phi_{1}^{+}, 1\right), \ldots,\left(\phi_{k}^{+}, 1\right),\left(g_{1}^{+}, 2 \mu_{1}^{+}\right), \ldots,\left(g_{r^{+}}^{+}, 2 \mu_{r^{+}}^{+}\right)\right\} .
$$

By construction $R^{+}\left(p_{i}\right) \cap \partial E^{*} \subseteq \operatorname{graph}\left(Z^{+}\right) \subseteq\left(\Lambda_{i-1}^{n}\right) \cap R^{+}\left(p_{i}\right)$. In addition the set of points of $\operatorname{graph}\left(Z^{+}\right)$ where $\eta_{Z^{+}}$is odd coincides with the set of points of $R^{+}\left(p_{i}\right)$ where $\theta_{\Lambda_{i-1}^{n}}$ is odd (which coincides, in turn, with $R^{+}\left(p_{i}\right) \cap \partial E^{*}$, possibly with the exclusion of $\left.p_{i}\right)$.

If $R^{-}\left(p_{i}\right) \cap \partial E^{*} \supseteqq\left\{p_{i}\right\}$ we repeat the same construction in $R^{-}\left(p_{i}\right)$.

As $\partial R\left(p_{i}\right) \subset \operatorname{Reg}_{\Gamma} \subseteq \operatorname{Reg}_{\Lambda_{i-1}^{n}}$ and $\eta_{\widehat{Y}^{ \pm}}=\theta_{\Lambda_{i-1}^{n}}$, we have that $\eta_{Z^{+}}=\theta_{\Lambda_{i-1}^{n}}$ on $(\{a\} \times[-b, b]) \cap\left(\Lambda_{i-1}^{n}\right)$ (respectively $\eta_{Z^{-}}=\theta_{\Lambda_{i-1}^{n}}$ on $\left.(\{-a\} \times[-b, b]) \cap\left(\Lambda_{i-1}^{n}\right)\right)$, so we can apply Lemma 4.9 and find a system of curves in $H^{2, p}(\mathbb{S})$, which will be our $\Lambda_{i}^{n}$, such that $\left(\Lambda_{i}^{n}\right) \cap R^{ \pm}\left(p_{i}\right)=\operatorname{graph}\left(Z^{ \pm}\right)$and $\theta_{\left.\Lambda_{i}^{n}\right|_{R^{ \pm}\left(p_{i}\right)}}=\eta_{Z^{ \pm}}$, while $\left(\Lambda_{i}^{n}\right)=\left(\Lambda_{i-1}^{n}\right)$ and $\theta_{\Lambda_{i}^{n}}=\theta_{\Lambda_{i-1}^{n}}$ outside of $R\left(p_{i}\right)$.

By construction we have that (i) and (ii) are satisfied. Furthermore

$$
\sup _{R\left(p_{i}\right)} \theta_{\Lambda_{i}^{n}}=\theta_{\Lambda_{i}^{n}}\left(p_{i}\right)=\theta_{\Lambda_{i-1}^{n}}\left(p_{i}\right)=\sup _{R\left(p_{i}\right)} \theta_{\Lambda_{i-1}^{n}}=\cdots=\sup _{R\left(p_{i}\right)} \theta_{\Lambda_{0}^{n}}=\sup _{R\left(p_{i}\right)} \theta_{\Gamma} \leqslant M .
$$

Since $\left(\Lambda_{i}^{n}\right) \subseteq\left(\Lambda_{i-1}^{n}\right) \subseteq(\Gamma)$, we get, using $(46)$,

$$
\mathcal{F}\left(\Lambda_{i}^{n},\left(\Lambda_{i}^{n}\right)^{-1}\left(R\left(p_{i}\right)\right)\right) \leqslant M \mathcal{F}\left(\Gamma, \Gamma^{-1}\left(R\left(p_{i}\right)\right)\right) \leqslant \frac{1}{C 2^{n}},
$$

and (iii) follows.

We now consider the most difficult case.

Case 2 of step 1 . Suppose $p_{i} \in \operatorname{acc} \operatorname{sing}\left(\Lambda_{i-1}^{n}\right) \cap \operatorname{Reg}_{\partial E^{*}}$.

We keep the notation introduced at the beginning of step 1, but we omit the super/subscript \pm , since we directly work on the whole of $R\left(p_{i}\right)$.

Using the assumption that $p_{i}$ is a regular point for $\partial E^{*}$ it follows that

$$
R\left(p_{i}\right) \cap \partial E^{*}=\operatorname{graph}(\phi),
$$

where $\phi \in H^{2, p}(]-a, a[)$, and $\phi(0)=\phi^{\prime}(0)=0$. Let $\left\{z_{1}^{ \pm}, \ldots, z_{j^{ \pm}}^{ \pm}\right\}:=(\{ \pm a\} \times[-b, b]) \cap\left(\Lambda_{i-1}^{n}\right)$. Fix $f_{l}$ with $1 \leqslant l \leqslant r$. Let

$$
I_{l}:=\left\{x \in[-a, a]: f_{l}(x) \neq \phi(x)\right\}=\bigcup_{k \in \mathbb{N}} I_{l k},
$$


where the $I_{l k}$ are open pairwise disjoint intervals. We replace the function $f_{l}$ with the function $g_{l}$ defined as follows. If $I_{l}$ is composed by a finite number of connected components then we let $g_{l}:=f_{l}$. Otherwise, let $\sigma \in \mathbb{N}$ and define $g_{l, \sigma} \in H^{2, p}(]-a, a[)$ as

$$
g_{l, \sigma}:= \begin{cases}\phi & \text { on } I_{l k} \text { with } k \geqslant \sigma \text { and } \pm a \notin \partial I_{l k}, \\ f_{l} & \text { otherwise in }[-a, a] .\end{cases}
$$

The requirement $\pm a \notin \partial I_{l k}$ is needed to ensure that the conditions on the lateral boundary of $R\left(p_{i}\right)$ remain unchanged.

Observe that $g_{l, \sigma}^{\prime}=f_{l}^{\prime}$ on $[-a, a] \backslash \bigcup_{k \geqslant \sigma} I_{l k}$ and $g_{l, \sigma}^{\prime \prime}=f_{l}^{\prime \prime}$ almost everywhere on $[-a, a] \backslash \bigcup_{k \geqslant \sigma} I_{l k}$. Since $\lim _{\sigma \rightarrow+\infty} \sum_{k \geqslant \sigma} \mathcal{H}^{1}\left(I_{l k}\right)=0$, using the absolute continuity of the Lebesgue integral, we can choose $\sigma_{l}(n) \in \mathbb{N}$ such that

$$
\left|\mathcal{P}\left(f_{l},\right]-a, a[)-\mathcal{P}\left(g_{l, \sigma_{l}(n)},\right]-a, a[)\right| \leqslant \mathcal{P}\left(f_{l}, \bigcup_{k \geqslant \sigma_{l}(n)} I_{l k}\right)+\mathcal{P}\left(\phi, \bigcup_{k \geqslant \sigma_{l}(n)} I_{l k}\right) \leqslant \frac{1}{2^{n} \delta(n) r} .
$$

Repeating the same arguments for every $l \in\{1, \ldots, r\}$ we obtain a collection of functions $\left\{g_{1}, \ldots, g_{r}\right\} \subset$ $H^{2, p}(]-a, a[)$ defined as $g_{i}:=g_{i, \sigma_{i}(n)}$. Let us consider the family

$$
Z:=\left\{(\phi, 1),\left(g_{1}, 2 \mu_{1}\right), \ldots,\left(g_{r}, 2 \mu_{r}\right)\right\}
$$

By construction we have $R\left(p_{i}\right) \cap \partial E^{*} \subseteq \operatorname{graph}(Z) \subseteq\left(\Lambda_{i-1}^{n}\right) \cap R\left(p_{i}\right), \operatorname{graph}(Z) \cap \partial R\left(p_{i}\right)=\left\{z_{1}^{ \pm}, \ldots, z_{j}^{ \pm}\right\}$and $\eta_{Z}=\theta_{\Lambda_{i-1}^{n}}$ on $\partial R\left(p_{i}\right)$. Applying Lemma 4.9 we obtain a system of curves in $H^{2, p}(\mathbb{S})$, which will be our $\Lambda_{i}^{n}$, whose trace and density function outside $R\left(p_{i}\right)$ are the same of $\Lambda_{i-1}^{n}$, while $\left(\Lambda_{i}^{n}\right) \cap R\left(p_{i}\right)=\operatorname{graph}(Z)$ and $\theta_{\Lambda_{i}^{n}}=\eta_{Z}$ on $\operatorname{graph}(Z)$. By construction we have that (ii) is satisfied; moreover $\{q \in \operatorname{graph}(Z): \eta(q)$ is odd $\}=\operatorname{graph}(\phi)$. Hence (i) is valid. Using (49) we obtain

$$
\begin{aligned}
& \left|\mathcal{F}\left(\Lambda_{i}^{n},\left(\Lambda_{i}^{n}\right)^{-1}\left(R\left(p_{i}\right)\right)\right)-\mathcal{F}\left(\Lambda_{i-1}^{n},\left(\Lambda_{i-1}^{n}\right)^{-1}\left(R\left(p_{i}\right)\right)\right)\right| \\
& \quad=\left|\left(\mathcal{P}(\phi,]-a, a[)+\sum_{l=1}^{r} 2 \mu_{l} \mathcal{P}\left(g_{l},\right]-a, a[)\right)-\left(\mathcal{P}(\phi,]-a, a[)+\sum_{l=1}^{r} 2 \mu_{l} \mathcal{P}\left(f_{l},\right]-a, a[)\right)\right| \\
& \quad \leqslant M \sum_{l=1}^{r}\left|\mathcal{P}\left(g_{l},\right]-a, a[)-\mathcal{P}\left(f_{l},\right]-a, a[)\right| \leqslant \frac{M}{2^{n} \delta(n)} .
\end{aligned}
$$

Hence (iii) is valid and this concludes the proof in case 2.

We are now in a position to conclude the proof of step 1 . Define

$$
\Lambda_{n}:=\Lambda_{\delta(n)}^{n} .
$$

By construction we have $\partial E^{*} \subseteq\left(\Lambda_{n}\right) \subseteq(\Gamma)$ and $\sharp\left(\operatorname{Sing}_{\Lambda_{n}} \cap \partial E^{*}\right)<+\infty$. Furthermore since the set of all points where $\Lambda_{n}$ has odd density is the same as the set of all points where $\Gamma$ has odd density, from Proposition 3.13, we obtain that $\left|A_{\Lambda_{n}}^{o} \Delta E^{*}\right|=0$. Therefore (a), (b) and (c) hold.

Since by construction the support and density function of $\Lambda_{i}^{n}$ and $\Lambda_{i-1}^{n}$ agree outside of $R\left(p_{i}\right)$, we have, using also (iii),

$$
\begin{aligned}
\left|\mathcal{F}\left(\Lambda_{n}\right)-\mathcal{F}(\Gamma)\right| & =\left|\mathcal{F}\left(\Lambda_{\delta(n)}^{n}\right)-\mathcal{F}\left(\Lambda_{0}^{n}\right)\right| \leqslant \sum_{i=1}^{\delta(n)}\left|\mathcal{F}\left(\Lambda_{i}^{n}\right)-\mathcal{F}\left(\Lambda_{i-1}^{n}\right)\right| \\
& =\sum_{i=1}^{\delta(n)}\left|\mathcal{F}\left(\Lambda_{i}^{n},\left(\Lambda_{i}^{n}\right)^{-1}\left(R\left(p_{i}\right)\right)\right)-\mathcal{F}\left(\Lambda_{i-1}^{n},\left(\Lambda_{i-1}^{n}\right)^{-1}\left(R\left(p_{i}\right)\right)\right)\right|
\end{aligned}
$$




$$
\begin{aligned}
= & \sum_{i: p_{i} \in \operatorname{Sing}_{\partial E^{*}}}\left|\mathcal{F}\left(\Lambda_{i}^{n},\left(\Lambda_{i}^{n}\right)^{-1}\left(R\left(p_{i}\right)\right)\right)-\mathcal{F}\left(\Lambda_{i-1}^{n},\left(\Lambda_{i-1}^{n}\right)^{-1}\left(R\left(p_{i}\right)\right)\right)\right| \\
& +\sum_{i: p_{i} \in \operatorname{Reg}_{\partial E^{*}}}\left|\mathcal{F}\left(\Lambda_{i}^{n},\left(\Lambda_{i}^{n}\right)^{-1}\left(R\left(p_{i}\right)\right)\right)-\mathcal{F}\left(\Lambda_{i-1}^{n},\left(\Lambda_{i-1}^{n}\right)^{-1}\left(R\left(p_{i}\right)\right)\right)\right| \leqslant \frac{1+M}{2^{n}} .
\end{aligned}
$$

Hence also (d) is valid, and the proof of step 1 is concluded.

Step 2. We construct a sequence $\left\{\Gamma_{n}\right\} \subset H^{2, p}(\mathbb{S})$ of limit systems of curves such that

(a') $\partial E^{*} \subseteq\left(\Gamma_{n}\right) \subseteq\left(\Lambda_{n}\right)$;

(b') $\left|A_{\Gamma_{n}} \Delta A_{\Lambda_{n}}^{o}\right|=0$;

(c') $\operatorname{Sing}_{\Gamma_{n}} \cap\left(\mathbb{R}^{2} \backslash \partial E^{*}\right)$ is a finite set for any $n \in \mathbb{N}$;

$\left(\mathrm{d}^{\prime}\right) \mathcal{F}\left(\Gamma_{n}\right) \leqslant \mathcal{F}\left(\Lambda_{n}\right)$ for any $n \in \mathbb{N}$.

In order to construct $\Gamma_{n}$ we use a recursive algorithm consisting of $\delta(n)$ steps. Let $\Gamma_{0}^{n}:=\Lambda_{n}, 1 \leqslant i \leqslant \delta(n)$ and suppose that $\Gamma_{i-1}^{n}$ has been defined. Then $\Gamma_{i}^{n}$ is obtained by modifying $\Gamma_{i-1}^{n}$ only on $\operatorname{int}\left(R\left(p_{i}\right)\right)$, in such a way that:

(i') the set of the points of $\left(\Gamma_{i}^{n}\right)$ where $\theta_{\Gamma_{i}^{n}}$ is odd is the same as the set of the points of $\left(\Gamma_{i-1}^{n}\right)$ where $\theta_{\Gamma_{i-1}^{n}}$ is odd;

(ii') $R\left(p_{i}\right) \cap \operatorname{Sing}_{\Gamma_{i}^{n}} \cap\left(\mathbb{R}^{2} \backslash \partial E^{*}\right)$ is finite;

(iii') $\mathcal{F}\left(\Gamma_{i}^{n},\left(\Gamma_{i}^{n}\right)^{-1}\left(R\left(p_{i}\right)\right)\right) \leqslant \mathcal{F}\left(\Gamma_{i-1}^{n},\left(\Gamma_{i-1}^{n}\right)^{-1}\left(R\left(p_{i}\right)\right)\right)$.

We proceed in two different ways depending on whether $p_{i} \in \partial E^{*}, p_{i} \in \mathbb{R}^{2} \backslash \partial E^{*}$.

Case 1 of step 2. Suppose $p_{i} \in \partial E^{*}$.

Repeating the construction at the beginning of step 1 we can find a family

$$
G^{+}:=\left\{\left(\phi_{1}^{+}, 1\right), \ldots,\left(\phi_{k}^{+}, 1\right),\left(u_{1}^{+}, 2 v_{1}^{+}\right), \ldots,\left(u_{r^{+}}^{+}, 2 v_{r^{+}}^{+}\right)\right\} \subset H^{2, p}(] 0, a[) \times(\mathbb{N} \backslash\{0\}),
$$

such that the functions $u_{i}$ are all distinct and $\phi_{i}^{+}<\phi_{j}^{+}$on $\left.] 0, a\right]$ for $i<j$, and

$$
\begin{aligned}
& R^{+}\left(p_{i}\right) \cap \partial E^{*}=\bigcup_{l=1}^{k} \operatorname{graph}\left(\phi_{l}^{+}\right) ; \\
& R^{+}\left(p_{i}\right) \cap\left(\Gamma_{i-1}^{n}\right)=\operatorname{graph}\left(G^{+}\right) ; \\
& \eta_{G^{+}}=\theta_{\Gamma_{i-1}^{n}} \quad \text { in } R^{+}\left(p_{i}\right) .
\end{aligned}
$$

Notice that if $p_{i} \in \operatorname{Sing}_{\partial E^{*}}$ (respectively $p_{i} \in \operatorname{Reg}_{\partial E^{*}}$ ) the function $\phi_{l}^{+}$coincides with the function $\phi_{l}^{+}$of case 1 of step 1 (respectively $k=1$ and $\phi_{1}^{+}$coincides with $\phi_{\mid R^{+}\left(p_{i}\right)}$ where $\phi$ is the function of case 2 of step 1 ).

We want to modify $\left(\Gamma_{i-1}^{n}\right) \cap R\left(p_{i}\right)$ leaving the functions $\phi_{l}^{+}$unchanged in order to fulfill (i'), while, to obtain (ii'), (iii'), we want to replace every $u_{l}^{+}$with a function $v_{l}^{+}$whose graph has energy lower than the energy of the graph of $u_{l}^{+}$and the graphs of the $v_{l}^{+}$intersect each other tangentially and only a finite number of times.

To this aim we let

$$
\Sigma_{l}^{+}:=\left\{v \in \mathcal{C}^{0}([0, a]): \operatorname{graph}(v) \subset \operatorname{graph}\left(G^{+}\right), v(a)=u_{l}^{+}(a)\right\}, \quad l \in\left\{1, \ldots, r^{+}\right\} .
$$

Applying Lemma 4.5 we obtain a family $\left\{v_{1}^{+}, \ldots, v_{r^{+}}^{+}\right\} \subset H^{2, p}(] 0, a[)$ such that $v_{l}^{+}$is a minimizer for $\mathcal{P}$ in $\Sigma_{l}^{+}$ and if $v_{l}^{+}(c)=v_{j}^{+}(c)$ for some $\left.\left.c \in\right] 0, a\right]$ then $v_{l}^{+} \equiv v_{j}^{+}$in $[0, c]$. Then we consider the family

$$
H^{+}:=\left\{\left(\phi_{1}^{+}, 1\right), \ldots,\left(\phi_{k}^{+}, 1\right),\left(v_{1}^{+}, 2 v_{1}^{+}\right), \ldots,\left(v_{r^{+}}^{+}, 2 v_{r^{+}}^{+}\right)\right\} \subset H^{2, p}(] 0, a[) \times(\mathbb{N} \backslash\{0\}) .
$$


We remark that $\left(\Gamma_{i-1}^{n}\right) \cap \partial R^{+}\left(p_{i}\right)=\operatorname{graph}\left(H^{+}\right) \cap \partial R^{+}\left(p_{i}\right)$. In addition, if $z \in \operatorname{graph}\left(H^{+}\right) \cap \partial R^{+}\left(p_{i}\right)$ then $\eta_{H^{+}}(z)=\eta_{G^{+}}(z)=\theta_{\Gamma_{i-1}^{n}}(z)$. Finally we note that, by construction, $\eta_{H^{+}}(q)$ is odd if and only if $q \in$ $\bigcup_{l=1}^{k} \operatorname{graph}\left(\phi_{l}^{+}\right)$(possibly with the exclusion of $p_{i}$ ).

Then we repeat the same construction in $R^{-}\left(p_{i}\right)$.

As all the hypotheses are fulfilled, we can apply Lemma 4.9 and obtain a system of curves $\Gamma_{i}^{n} \in H^{2, p}(\mathbb{S})$ such that $\left(\Gamma_{i}^{n}\right) \cap R^{ \pm}\left(p_{i}\right)=\operatorname{graph}\left(H^{ \pm}\right)$and $\left(\theta_{\Gamma_{i}^{n}}\right)_{\mid R^{ \pm}\left(p_{i}\right)}=\eta_{H^{ \pm}}$.

Since

$$
\left(\Gamma_{i}^{n}\right)^{-1}\left(\left(\mathbb{R}^{2} \backslash \partial E^{*}\right) \cap R^{ \pm}\left(p_{i}\right)\right) \subset \bigcup_{l=1}^{r^{ \pm}} \operatorname{graph}\left(v_{l}^{ \pm}\right)
$$

and the set of singular points of $\bigcup_{l=1}^{r^{ \pm}} \operatorname{graph}\left(v_{l}^{ \pm}\right)$is finite we have that $\Gamma_{i}^{n}$ satisfies (ii'). Furthermore

$$
\begin{aligned}
\mathcal{F} & \left(\Gamma_{i}^{n},\left(\Gamma_{i}^{n}\right)^{-1}\left(R\left(p_{i}\right)\right)\right) \\
& =\sum_{l=1}^{k^{-}} \mathcal{P}\left(\phi_{l}^{-},\right]-a, 0[)+\sum_{l=1}^{k^{+}} \mathcal{P}\left(\phi_{l}^{+},\right]-a, 0[)+\sum_{l=1}^{r^{-}} 2 v_{l}^{-} \mathcal{P}\left(v_{l}^{-},\right]-a, 0[)+\sum_{l=1}^{r^{+}} 2 v_{l}^{+} \mathcal{P}\left(v_{l}^{+},\right] 0, a[) \\
& \leqslant \sum_{l=1}^{k^{-}} \mathcal{P}\left(\phi_{l}^{-},\right]-a, 0[)+\sum_{l=1}^{k^{+}} \mathcal{P}\left(\phi_{l}^{+},\right]-a, 0[)+\sum_{l=1}^{r^{-}} 2 v_{l}^{-} \mathcal{P}\left(u_{l}^{-},\right]-a, 0[)+\sum_{l=1}^{r^{+}} 2 v_{l}^{+} \mathcal{P}\left(u_{l}^{+},\right] 0, a[) \\
& =\mathcal{F}\left(\Gamma_{i-1}^{n},\left(\Gamma_{i-1}^{n}\right)^{-1}\left(R\left(p_{i}\right)\right)\right),
\end{aligned}
$$

which is (iii').

Case 2 of step 2. Suppose $p_{i} \in \mathbb{R}^{2} \backslash \partial E^{*}$.

In this case we obtain $\Gamma_{i}^{n}$ simply repeating the construction used in step 1 in the proof of Theorem 5.1. Since we supposed that $R\left(p_{i}\right) \Subset \mathbb{R}^{2} \backslash \partial E^{*}$, in $R\left(p_{i}\right)$ there are not points of $(\Gamma)$ or of $\left(\Gamma_{i-1}^{n}\right)$ with odd density. Therefore the set of points of $\left(\Gamma_{i}^{n}\right)$ where $\theta_{\Gamma_{i}^{n}}$ is odd coincides with the set of points of $\left(\Gamma_{i-1}^{n}\right)$ where $\theta_{\Gamma_{i-1}^{n}}$ is odd, by construction we have

$$
\mathcal{F}\left(\Gamma_{i}^{n},\left(\Gamma_{i}^{n}\right)^{-1}\left(R\left(p_{i}\right)\right)\right) \leqslant \mathcal{F}\left(\Gamma_{i-1}^{n},\left(\Gamma_{i-1}^{n}\right)^{-1}\left(R\left(p_{i}\right)\right)\right),
$$

and $\left(\Gamma_{i}^{n}\right)$ verifies the finiteness property in $R\left(p_{i}\right)$. This concludes the proof of case 2 of step 2.

We are now in a position to conclude the proof of step 2. Define

$$
\Gamma_{n}:=\Gamma_{\delta(n)}^{n} \text {. }
$$

Applying Theorem 5.1 we can find a limit system of curves which is equivalent to $\Gamma_{n}$. Let us still denote by $\Gamma_{n}$ this new limit system of curves. Since we did not modify the set of points with odd multiplicity, thanks to Proposition 3.13 we have that $\left|A_{\Gamma_{n}} \Delta A_{\Lambda_{n}}^{o}\right|=0$ which is the assertion of $\left(b^{\prime}\right)$. By construction we have $\partial E^{*} \subseteq$ $\left(\Gamma_{n}\right) \subseteq\left(\Lambda_{n}\right) \subseteq(\Gamma)$, and $\sharp\left(\operatorname{Sing}_{\Gamma_{n}} \cap\left(\mathbb{R}^{2} \backslash \partial E^{*}\right)\right)<+\infty$ which are the assertions of $\left(a^{\prime}\right)$ and $\left(c^{\prime}\right)$. Furthermore,

$$
\mathcal{F}\left(\Gamma_{n}\right)=\mathcal{F}\left(\Gamma_{\delta(n)}^{n}\right) \leqslant \mathcal{F}\left(\Gamma_{\delta(n)-1}^{n}\right) \leqslant \cdots \leqslant \mathcal{F}\left(\Gamma_{1}^{n}\right) \leqslant \mathcal{F}\left(\Gamma_{0}^{n}\right)=\mathcal{F}\left(\Lambda_{n}\right),
$$

which proves $\left(d^{\prime}\right)$ and this concludes the proof of step 2.

Now, using the properties of $\Lambda_{n}$ and $\Gamma_{n}$, we can conclude the proof of the theorem. Thanks to $(b),\left(b^{\prime}\right)$, we have

$$
\left|A_{\Gamma_{n}} \Delta E\right| \leqslant\left|A_{\Gamma^{n}} \Delta A_{\Lambda_{n}}^{o}\right|+\left|A_{\Lambda_{n}}^{o} \Delta E\right|=0,
$$

and hence (45) holds. Since every $\Gamma_{n}$ is a limit system of curves and (45) holds, using Remarks 2.23 and 2.20, we have $\left\{\Gamma_{n}\right\} \subset \mathcal{A}(E)$. Given $n \in \mathbb{N}$, from $(c)$ and $\left(c^{\prime}\right)$ it follows that $\left(\Gamma_{n}\right)$ verifies the finiteness property, therefore $\left\{\Gamma_{n}\right\} \subset \mathcal{Q}_{\text {fin }}(E)$. 
By construction we have $\left\|\theta_{\Gamma_{n}}\right\|_{L^{\infty}} \leqslant\left\|\theta_{\Gamma}\right\|_{L^{\infty}}$ and from $(a),\left(a^{\prime}\right)$ it follows that $\left(\Gamma_{n}\right) \subseteq(\Gamma)$, hence

$$
\sup _{n \in \mathbb{N}} \mathcal{F}\left(\Gamma_{n}\right)<+\infty \text {. }
$$

So we can apply Theorem 2.10 to obtain a subsequence (still indicated by $\left.\left\{\Gamma_{n}\right\}\right) H^{2, p}$-weakly converging to a certain system of curves $\widetilde{\Gamma}$. Again as in the proof of Theorem 5.1, due to the fact that the diameters of the nice rectangles used to cover the set $\operatorname{acc} \operatorname{sing}(\Gamma)$ uniformly decrease to 0 , we obtain

$$
\operatorname{Reg}_{\widetilde{\Gamma}}=\operatorname{Reg}_{\Gamma}, \quad\left(\theta_{\widetilde{\Gamma}}\right)_{\mid \operatorname{Reg}_{\widetilde{\Gamma}}}=\left(\theta_{\Gamma}\right)_{\mid \operatorname{Reg}_{\Gamma}} .
$$

These relations, together with Lemma 3.11, imply that $\widetilde{\Gamma} \sim \Gamma$.

Using $(d),\left(d^{\prime}\right)$, Lemma 3.9 and the $H^{2, p}$ weak lower semicontinuity of $\mathcal{F}$, we have

$$
\begin{aligned}
\mathcal{F}(\Gamma) & =\mathcal{F}(\widetilde{\Gamma}) \leqslant \liminf _{n \rightarrow+\infty} \mathcal{F}\left(\Gamma_{n}\right) \leqslant \limsup _{n \rightarrow+\infty} \mathcal{F}\left(\Gamma_{n}\right) \\
& \leqslant \limsup _{n \rightarrow+\infty} \mathcal{F}\left(\Lambda_{n}\right)=\lim _{n \rightarrow+\infty} \mathcal{F}\left(\Lambda_{n}\right)=\mathcal{F}(\Gamma) .
\end{aligned}
$$

Eventually, assertion (44) is a direct consequence of (42) and the assertions concerning $\left\{\Gamma_{n}\right\}$.

Fig. 7 illustrates the construction of the sequence $\left\{\Gamma_{n}\right\}$ of Theorem 6.3 in a particular situation.

\section{Regularity of minimal systems in $\mathcal{A}(E)$}

In the following theorem we prove a regularity result for minimal systems of curves. We limit ourselves to study the regularity of $(\Gamma)$ in $\mathbb{R}^{2} \backslash \partial E^{*}$ and locally around regular points, since we know that $\Gamma$ is without crossings and the optimal regularity for $(\Gamma)$ in $\operatorname{Reg}_{\Gamma} \cap \partial E^{*}$ is given by the local representation with functions of class $H^{2, p}$.

Theorem 7.1. Let $p=2$ and let $E \subset \mathbb{R}^{2}$ be such that $\overline{\mathcal{F}}(E)<+\infty$. Then every minimal system $\Gamma$ of curves in $\mathcal{A}(E)$ verifies the finiteness property in any open subset $U \Subset \mathbb{R}^{2} \backslash \partial E^{*}$.

Furthermore, every connected component $B$ of $\operatorname{Reg}_{\Gamma} \cap \bar{U}$ is an analytic curve and its curvature $\kappa$ verifies the equation

$$
2 \frac{d^{2}}{d s^{2}} \kappa+\kappa^{3}-\kappa=0, \quad s \in\left[0, \mathcal{H}^{1}(B)\right]
$$

Proof. Let $\Gamma$ be a minimal system in $\mathcal{A}(E)$ and fix an open set $U \Subset \mathbb{R}^{2} \backslash \partial E^{*}$. The proof consists of two steps.

Step 1 . Every connected component $B$ of $\operatorname{Reg}_{\Gamma} \cap \bar{U}$ is an analytic curve and its curvature $\kappa$ verifies (52).

Let $B$ be a connected component of $\operatorname{Reg}_{\Gamma} \cap \bar{U} ; B$ is a one-dimensional submanifold of $\mathbb{R}^{2}$ of class $H^{2,2}$ and $\theta_{\Gamma}$ is constant and even on $B$. Let $\alpha:\left[0, \mathcal{H}^{1}(B)\right] \rightarrow \mathbb{R}^{2}$ be a parametrization by arc length of $B$, let $\eta \in C_{c}^{\infty}\left(\left[0, \mathcal{H}^{1}(B)\right]\right)$ and consider the curve $\alpha_{\varepsilon}:\left[0, \mathcal{H}^{1}(B)\right] \rightarrow \mathbb{R}^{2}, \alpha_{\varepsilon}:=\alpha+\varepsilon \eta$. For $|\varepsilon| \ll 1$ we have $\left(\alpha_{\varepsilon}\right) \cap$ $[(\Gamma) \backslash B]=\emptyset$. Using Lemma 4.1, we can find a system of curves $\Gamma_{\varepsilon} \in \mathcal{A}(E)$, whose trace is given by

$$
\left(\Gamma_{\varepsilon}\right)=[(\Gamma) \backslash B] \cup\left(\alpha_{\varepsilon}\right),
$$

and $\theta_{\Gamma_{\varepsilon}}=\theta_{\Gamma}$ on $(\Gamma) \backslash B$, while $\theta_{\Gamma_{\varepsilon}}$ on $\left(\alpha_{\varepsilon}\right)$ assumes the same constant value of $\theta_{\Gamma}$ on $B$. Since the set of points with odd multiplicity of $\Gamma$ and $\Gamma_{\varepsilon}$ coincides, using Corollary 5.2 and Proposition 3.13, we can find a system of curves $\widetilde{\Gamma}_{\varepsilon} \in \mathcal{A}(E)$ which is equivalent to $\Gamma_{\varepsilon}$. Therefore, from the minimality of $\Gamma$ on $\mathcal{A}(E)$, we have

$$
\lim _{\varepsilon \rightarrow 0} \frac{\mathcal{F}(\alpha+\varepsilon \eta)-\mathcal{F}(\alpha)}{\varepsilon}=0
$$


Using [13] and the regularity theory of ordinary differential equations, it follows that $B$ is an analytic submanifold of $\mathbb{R}^{2}$ and (52) holds.

Step 2. $\Gamma$ verifies the finiteness property in $U$.

Suppose by contradiction that there exists $U \Subset \mathbb{R}^{2} \backslash \partial E^{*}$ such that $\Gamma$ does not verify the finiteness property in $U$. Hence accsing $(\Gamma) \cap \bar{U} \neq \emptyset$. Let $p \in \operatorname{acc} \operatorname{Sing}(\Gamma) \cap \bar{U}$ and let $R(p)=[-a, a] \times[-b, b]$ be a nice rectangle for $(\Gamma)$ at $p$ such that

$$
\begin{aligned}
& R(p) \Subset \mathbb{R}^{2} \backslash \partial E^{*} ; \\
& \sharp\left(R^{+}(p) \cap \operatorname{Sing}_{\Gamma}\right)=+\infty ; \\
& (\Gamma) \cap \partial R(p) \subset \operatorname{Reg}_{\Gamma} .
\end{aligned}
$$

Let

$$
Y^{+}:=\left\{\left(f_{1}^{+}, \mu_{1}^{+}\right), \ldots,\left(f_{r}^{+}, \mu_{r}^{+}\right)\right\} \subset H^{2, p}(] 0, a[) \times(2 \mathbb{N} \backslash\{0\})
$$

be a canonical family for $\Gamma$ in $R^{+}(p)$. For every $i, j \in\{1, \ldots, r\}$ let

$$
I_{i j}:=\left\{x \in[0, a]:\left(x, f_{i}^{+}(x)\right)=\left(x, f_{j}^{+}(x)\right) \in \operatorname{Sing}_{\Gamma}\right\}, \quad \xi_{i j}:=\sup \left\{x: x \in I_{i j}\right\} .
$$

Note that, since $(\Gamma) \cap \partial R(p) \subset \operatorname{Reg}_{\Gamma}$, we have $a \notin I_{i j}$. If $0<\xi_{i j}<a$ then, due to the minimality of $\Gamma$ in $\mathcal{A}(E)$ we have

$$
\mathcal{P}\left(f_{i}^{+},\right] 0, \xi_{i j}[)=\mathcal{P}\left(f_{j}^{+},\right] 0, \xi_{i j}[) .
$$

Indeed, suppose by contradiction that $\mathcal{P}\left(f_{i}^{+},\right] 0, \xi_{i j}[)<\mathcal{P}\left(f_{j}^{+},\right] 0, \xi_{i j}[)$. Setting

$$
\widehat{f}_{j}^{+}:= \begin{cases}f_{i}^{+} & \text {on }\left[0, \xi_{i j}\right], \\ f_{j}^{+} & \text {on }\left[\xi_{i j}, a\right],\end{cases}
$$

from Lemma 4.9 and Corollary 5.2, we can find a system of curves $\widehat{\Gamma} \in \mathcal{A}(E)$ such that $(\widehat{\Gamma}) \cap R^{+}(p)=$ $\operatorname{graph}(\widehat{Y}), \theta_{\widehat{\Gamma} \mid R^{+}(p)}=\eta_{\widehat{Y}}$, where $\widehat{Y}$ is obtained from $Y$ by replacing $f_{j}$ with $\widehat{f}_{j}$. Hence $\mathcal{F}(\widehat{\Gamma})<\mathcal{F}(\Gamma)$ which contradicts the minimality of $\Gamma$, and (53) is proved.

Using step 1 we have that each $f_{i}^{+}$is analytic on the interval $\left[0, \widetilde{\xi}_{i}\right]$, where $\widetilde{\xi}_{i}:=\sup \left\{\xi_{i j}: 1 \leqslant j \leqslant r\right\}$. Therefore, if $I_{i j}$ is infinite then $f_{i}^{+}=f_{j}^{+}$on $\left[0, \xi_{i j}\right]$. It follows that $\Gamma$ verifies the finiteness property in $R^{+}(p)$ and we have a contradiction.

Remark 7.2. In the general case $p \in] 1,+\infty[, p \neq 2$, arguing as in the proof of Theorem 5.1, for a fixed $U \Subset \mathbb{R}^{2} \backslash \partial E^{*}$ it is possible to prove that there exists a minimal system of curves $\Gamma$ in $\mathcal{A}(E)$ verifying the finiteness property in $U$. The Euler equation for a functional whose integrand is a smooth function of $\kappa$ can be found in the literature, see for instance [13, pages 63, 64]. In this case a regularity result (similar to the one in Theorem 7.1) holds, at least on compact subsets where the curvature does not vanish.

\section{Characterization of the sets $E$ with $\overline{\mathcal{F}}(E)<+\infty$ and finite singular set}

As a consequence of Theorem 5.1 and Corollary 5.2 we have that any $E \subset \mathbb{R}^{2}$ with $\overline{\mathcal{F}}(E)<+\infty$ can be approximated, both in $L^{1}\left(\mathbb{R}^{2}\right)$ and in energy, with sets having a finite number of singular points. Our purpose is to give a characterization of the subsets of $\mathbb{R}^{2}$ with finite singular set (and finite relaxed energy). Throughout this section we will always suppose that $E \subset \mathbb{R}^{2}$ has continuous unoriented tangent, that $\partial E$ is piecewise $H^{2, p}$ and that $\operatorname{Sing}_{\partial E}$ is finite. Hence $E^{*}=E$ and for every $p \in \operatorname{Reg}_{\partial E}$ the set $E$ can be locally written as the subgraph of an $H^{2, p}$ function defined on $T_{p}(\partial E)$. 
The following definition is contained in [4, p. 282] and is needed to "count" the number of singularities of $\partial E$ with an appropriate multiplicity.

Definition 8.1. For every $p \in \operatorname{Sing}_{\partial E}$ we define the balanced multiplicity $\omega_{\partial E}(p)$ as

$$
\omega_{\partial E}(p):=\frac{\left|\rho^{+}(p)-\rho^{-}(p)\right|}{2},
$$

where $\rho^{+}(p)$ (respectively $\rho^{-}(p)$ ) is the number of distinct graphs necessary to cover $B_{r}^{+}(p) \cap \partial E$ (respectively $\left.B_{r}^{-}(p) \cap \partial E\right)$, for $r>0$ small enough.

Remark 8.2. As observed in [4, p. 282], $\left|\rho^{+}(p)-\rho^{-}(p)\right|$ is even for any $p \in \operatorname{Nod}_{\partial E}=\operatorname{Sing}_{\partial E}$. Indeed, given $p \in \operatorname{Nod}_{\partial E}$, there exists $r>0$ such that $B_{r}(p)$ contains both points of $E$ and of $\mathbb{R}^{2} \backslash \bar{E}$, the intersection between $\partial B_{r}(p)$ and $\partial E$ is transversal and hence the number of the elements of $\partial E \cap \partial B_{r}(p)$ is even. If $r$ is sufficiently small, this number coincides with $\rho^{+}(p)+\rho^{-}(p)$, which has the same parity of $\left|\rho^{+}(p)-\rho^{-}(p)\right|$.

The following result is contained in $[4$, Theorems $6.3,6.4]$.

Theorem 8.3. We have

$$
\sum_{p \in \operatorname{Sing}_{\partial E}} \omega(p) \text { is even } \Rightarrow \overline{\mathcal{F}}(E)<+\infty .
$$

Furthermore if $\operatorname{Sing}_{\partial E}=\left\{p_{1}, \ldots, p_{n}\right\}$ and $p_{i}$ is a simple cusp point for every $i=1, \ldots, n$, then

$$
\overline{\mathcal{F}}(E)<+\infty \Rightarrow n \text { is even. }
$$

Actually, a more refined result can be proved. Indeed, the following theorem holds.

Theorem 8.4. We have

$$
\overline{\mathcal{F}}(E)<+\infty \Rightarrow \sum_{p \in \operatorname{Sing}_{\partial E}} \omega_{\partial E}(p) \text { is even }
$$

Theorem 8.4 is based, among other tools, on formula (44) and on Theorem 8.3; since no new techniques are needed, we omit its proof, which can be found in [5].

We now want to prove Theorem 8.6, which is one of the main representation results for $\overline{\mathcal{F}}$ of the paper.

Let $E \subset \mathbb{R}^{2}$ be such that $\overline{\mathcal{F}}(E)<+\infty$. Suppose that $\operatorname{Sing}_{\partial E}$ is not empty, finite and composed only by simple cusp points. Using Theorem 8.3 we have

$$
\operatorname{Sing}_{\partial E}=\left\{p_{1}, p_{2}, \ldots, p_{2 M}\right\}, \quad M \in \mathbb{N} \backslash\{0\} .
$$

For every $p_{i} \in \operatorname{Sing}_{\partial E}$ we choose a unit vector $v\left(p_{i}\right)$ normal to $T_{p_{i}}(\partial E)$ in such a way that $\rho_{\partial E}^{+}\left(p_{i}\right) \geqslant \rho_{\partial E}^{-}\left(p_{i}\right)$. Accordingly, the half-nice rectangle $R^{+}\left(p_{i}\right)$ corresponds to $\rho^{+}\left(p_{i}\right)$.

Definition 8.5. Let $E \subset \mathbb{R}^{2}$ be as above. We define $\Sigma(E)$ as the set of all collections $\left\{\sigma_{1}, \ldots, \sigma_{M}\right\}$ of curves such that

(i) $\sigma_{i} \in H^{2, p}(0,1)$ and $\left|d \sigma_{i} / d t\right|$ is constant for every $i=1, \ldots, M$;

(ii) if $\sigma_{i}\left(t_{1}\right)=\sigma_{j}\left(t_{2}\right)$ for some $t_{1}, t_{2} \in[0,1]$ then $d \sigma_{i}\left(t_{1}\right) / d t$ and $d \sigma_{j}\left(t_{2}\right) / d t$ are parallel; moreover if $\sigma_{i}(t) \in \partial E$ for some $t \in[0,1]$, then $d \sigma_{i}(t) / d t$ is parallel to $T_{\sigma_{i}(t)}(\partial E)$;

(iii) $\sigma_{i}(0), \sigma_{i}(1) \in \operatorname{Sing}_{\partial E}$ for every $i \in\{1, \ldots, M\}$, and there exists a bijective application between $\left\{\sigma_{1}(0), \sigma_{1}(1)\right.$, $\left.\ldots, \sigma_{M}(0), \sigma_{M}(1)\right\}$ and $\operatorname{Sing}_{\partial E}$; 
(iv) for every $i \in\{1, \ldots, M\}$

$$
\begin{aligned}
& \frac{d \sigma_{i}}{d t}(0) \text { is parallel to } T_{\sigma_{i}(0)}(\partial E) \text { and points in the direction of } R^{-}\left(\sigma_{i}(0)\right), \\
& \frac{d \sigma_{i}}{d t}(1) \text { is parallel to } T_{\sigma_{i}(1)}(\partial E) \text { and points in the direction of } R^{+}\left(\sigma_{i}(1)\right) \text {. }
\end{aligned}
$$

It is immediate to see that the set $\Sigma(E)$ is not empty.

Theorem 8.6. Assume that $\overline{\mathcal{F}}(E)<+\infty$ and that $\operatorname{Sing}_{\partial E}$ consists of a finite number of simple cusp points $\left\{p_{1}, \ldots, p_{2 M}\right\}$. Then we have the following representation formula for $\overline{\mathcal{F}}(E)$ :

$$
\overline{\mathcal{F}}(E)=\int_{\operatorname{Reg}_{\partial E}}\left[1+\left|\kappa_{\partial E}\right|^{p}\right] d \mathcal{H}^{1}+2 \min _{\sigma \in \Sigma(E)} \mathcal{F}(\sigma) .
$$

Proof. The proof is divided into three steps.

Step 1 . We have

$$
\overline{\mathcal{F}}(E) \geqslant \int_{\operatorname{Reg}_{\partial E}}\left[1+\left|\kappa_{\partial E}\right|^{p}\right] d \mathcal{H}^{1}+2 \inf _{\sigma \in \Sigma(E)} \mathcal{F}(\sigma) .
$$

Thanks to (44), to obtain (56) it is enough to prove that for every $\Gamma \in \mathcal{Q}_{\text {fin }}(E)$ we can find $\sigma_{\Gamma}=\left\{\sigma_{1}, \ldots, \sigma_{M}\right\} \in$ $\Sigma(E)$ such that

$$
\mathcal{F}(\Gamma) \geqslant \int_{\operatorname{Reg}_{\partial E}}\left[1+\left|\kappa_{\partial E}\right|^{p}\right] d \mathcal{H}^{1}+2 \mathcal{F}\left(\sigma_{\Gamma}\right) .
$$

We will see that $\sigma_{\Gamma}$ satisfies also $\left(\sigma_{\Gamma}\right) \subset(\Gamma)$.

Let $\Gamma \in \mathcal{Q}_{\text {fin }}(E)$. For every $q \in \operatorname{Sing}_{\Gamma} \supseteq \operatorname{Sing}_{\partial E}$, we denote by $R(q)$ a nice rectangle for $\Gamma$ at $q$ such that

$$
R(q) \cap \operatorname{Sing}_{\Gamma}=\{q\} .
$$

Moreover for every $q \in \operatorname{Sing}_{\Gamma} \backslash \operatorname{Sing}_{\partial E}$ we make an arbitrary choice of a normal unit vector to $(\Gamma)$ at $q$ so that $R^{+}(q), R^{-}(q)$ are defined.

Let us construct $\sigma_{1}$. From now on with the symbol $\delta_{j}$ we denote +1 or -1 . Accordingly, for every $q \in \operatorname{Sing}_{\Gamma}$ we write $R^{\delta_{j}}(q)$ in place of $R^{ \pm}(q)$ when $\delta_{j}= \pm 1$.

Construction of $\sigma_{1}$. Set $\left(\mathcal{G}_{0}, \Psi_{0},\left(q_{0}, \delta_{0}\right)\right):=\left((\Gamma), \theta_{\Gamma},\left(p_{1},+1\right)\right)$. Suppose we have defined $\left(\mathcal{G}_{i}, \Psi_{i},\left(q_{i}, \delta_{i}\right)\right)$ for some $i \geqslant 1$, with:

$$
\Psi_{i}: \mathcal{G}_{i-1} \rightarrow \mathbb{N}, \quad \Psi_{i}:=\left\{\begin{array}{ll}
\Psi_{i-1}-2 & \text { on } H_{i}, \\
\Psi_{i-1} & \text { on } \mathcal{G}_{i-1} \backslash H_{i},
\end{array} \quad \mathcal{G}_{i}:=\left\{z \in \mathcal{G}_{i-1}: \Psi_{i}(z)>0\right\},\right.
$$

where $H_{i} \subset \mathcal{G}_{i-1}$ is a connected component of $\operatorname{Reg}_{\mathcal{G}_{i-1}}$ such that: $\Psi_{i-1} \geqslant 2$ is constant on $H_{i} ; q_{i} \in \operatorname{Sing}_{\Gamma}$ is a point of the relative boundary of $H_{i}$ (which is composed either by $q_{i-1}$ itself, and in this case we understand that $q_{i-1}=q_{i}$, or by two points $\left.\left\{q_{i-1}, q_{i}\right\} \subset \operatorname{Sing}_{\Gamma}\right) ; H_{i}$ crosses $R^{-\delta_{i-1}}\left(q_{i-1}\right)$ and reaches $q_{i}$ crossing $R^{\delta_{i}}\left(q_{i}\right)$;

(b) the function $\Psi_{i}$ verifies the train tracks property in the rectangle $R(q)$ for every $q \in \mathcal{G}_{i} \cap\left(\operatorname{Sing}_{\Gamma} \backslash\left\{p_{1}, q_{i}\right\}\right)$;

(c) if $q_{i} \neq p_{1}$ we have

moreover

$$
\sum_{z \in \mathcal{G}_{i} \cap \partial R^{+}\left(p_{1}\right)} \Psi_{i}(z)=\sum_{z \in \mathcal{G}_{i} \cap \partial R^{-}\left(p_{1}\right)} \Psi_{i}(z)+2 ;
$$

$$
\delta_{i}= \pm 1 \quad \Rightarrow \quad \sum_{z \in \mathcal{G}_{i} \cap \partial R^{+}\left(q_{i}\right)} \Psi_{i}(z)=\sum_{z \in \mathcal{G}_{i} \cap \partial R^{-}\left(q_{i}\right)} \Psi_{i}(z) \mp 2
$$



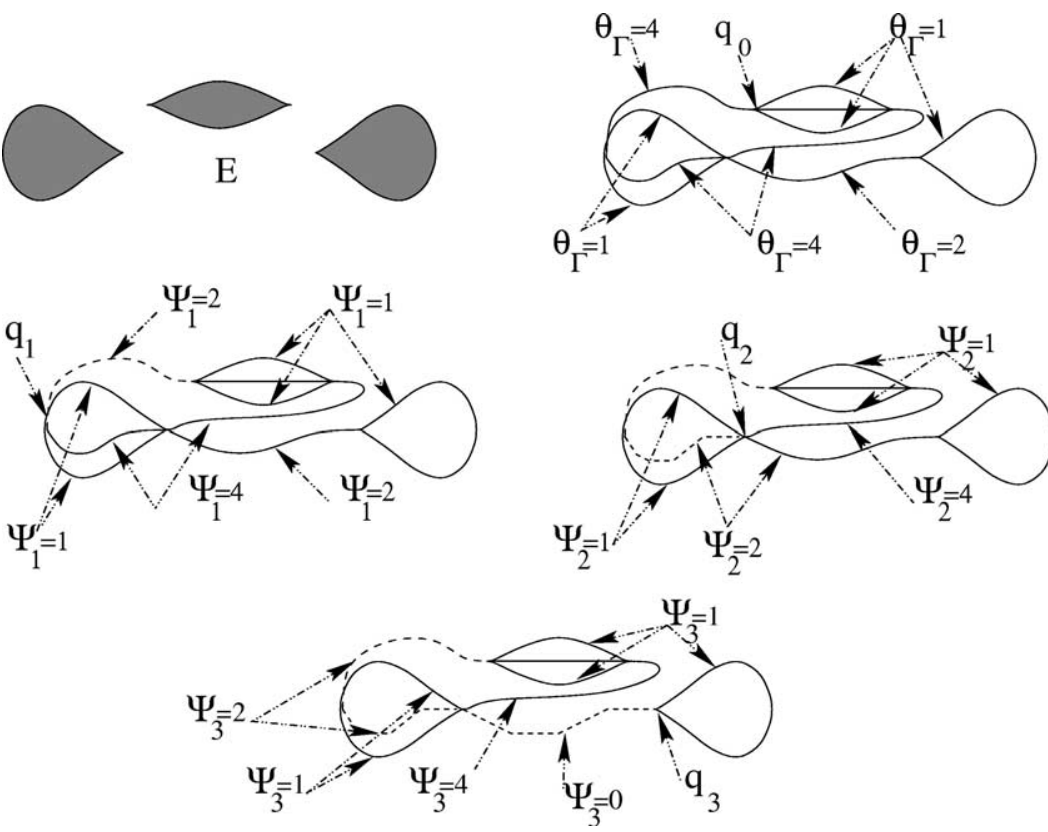

Fig. 9. The construction of $\sigma_{1}$ in step 1 in the proof of Theorem 8.6.

(d) if $q_{i}=p_{1}$ and $\delta_{i}=-1$ then

$$
\sum_{z \in \mathcal{G}_{i} \cap \partial R^{+}\left(p_{1}\right)} \Psi_{i}(z)=\sum_{z \in \mathcal{G}_{i} \cap \partial R^{-}\left(p_{1}\right)} \Psi_{i}(z)+4 ;
$$

(e) if $q_{i}=p_{1}$ and $\delta_{i}=+1$ then

$$
\sum_{z \in \mathcal{G}_{i} \cap \partial R^{+}\left(p_{1}\right)} \Psi_{i}(z)=\sum_{z \in \mathcal{G}_{i} \cap \partial R^{-}\left(p_{1}\right)} \Psi_{i}(z) .
$$

Let us construct the first step $(i=1)$. Since $p_{1}:=q_{0}$ is a simple cusp point and $\theta_{\Gamma}$ verifies the train tracks property, we can find a relative connected component of $\operatorname{Reg}_{\Gamma}$ which crosses $R^{-}\left(p_{1}\right)$ and over which $\Psi_{0}:=\theta_{\Gamma}$ is constant $\geqslant 2$. Hence we can define $\left(\mathcal{G}_{1}, \Psi_{1},\left(q_{1}, \delta_{1}\right)\right)$ satisfying properties (a)-(e), see Fig. 9.

Let us now explain in which way the algorithm constructs the step $i+1$ from the step $i \geqslant 1$. If $\{z \in$ $\left.\partial R^{-\delta_{i}}\left(q_{i}\right): \Psi_{i}(z) \geqslant 2\right\}=\emptyset$ then, from the hypothesis that $\operatorname{Sing}_{\partial E}$ is composed only by simple cusps and (d), (e), we have that $q_{i} \in \operatorname{Sing}_{\partial E} \backslash\left\{p_{1}\right\}$; in this case the algorithm stops and we set

$$
\left(\sigma_{1}\right):=\overline{H_{1}} \cup \cdots \cup \overline{H_{i}} .
$$

Otherwise, in view of (c), (d), (e) and (58) we can find an arc of regular points of $(\Gamma)$ which is contained in $\mathcal{G}_{i}$, crosses $R^{-\delta_{i}}\left(q_{i}\right)$ and is such that $\Psi_{i} \geqslant 2$ is constant on this arc. Let $H_{i+1}$ be the connected component of $\operatorname{Reg} \mathcal{G}_{i}$ containing this arc and let $\left\{q_{i}, q_{i+1}\right\}$ be the relative boundary of $H_{i+1}$ (possibly with $q_{i}=q_{i+1}$ ). Again from (b)-(e) we have that $\Psi_{i} \geqslant 2$ is constant on $H_{i+1}$ and $H_{i+1}$ reaches $q_{i+1}$ crossing $R^{\delta_{i+1}}\left(q_{i+1}\right)$. In addition, setting $\Psi_{i+1}$ and $\mathcal{G}_{i+1}$ as in (59) with $i$ replaced by $i+1$, we have, thanks to (58), that $\left(\mathcal{G}_{i+1}, \Psi_{i+1},\left(q_{i+1}, \delta_{i+1}\right)\right)$ satisfies (b)-(e) replacing everywhere $i$ with $i+1$, see Fig. 9 .

Therefore we can iterate the algorithm as specified above. Since $\Gamma \in \mathcal{Q}_{\text {fin }}(E)$ we have $\sharp \operatorname{Sing}_{\Gamma}<+\infty$, hence the algorithm stops after a finite number $n$ of steps. Furthermore $q_{n}=p_{j_{1}} \in \operatorname{Sing}_{\partial E} \backslash\left\{p_{1}\right\}$. Indeed, if by contradiction $q_{n}=p_{1}$, from (b)-(e) we could iterate the algorithm also at step $n+1$. 
We now define

$$
\left(\sigma_{1}\right):=\overline{H_{1}} \cup \cdots \cup \overline{H_{n}} .
$$

Since $H_{i}$ and $H_{i+1}$ have $q_{i}$ as a boundary point and belong to opposite half planes with respect to the normal line to $\mathcal{G}_{i}$ at $q_{i}$, using Lemma 4.1 we can find $\sigma_{1} \in H^{2, p}(0,1)$ parametrized with constant speed and such that $\left(\sigma_{1}\right)=\bigcup_{i=1}^{n} \overline{H_{i}} \subset(\Gamma), \sigma_{1}(0)=p_{1}, \sigma_{1}(1)=p_{j_{1}}$.

Construction of $\sigma_{2}$. In order to obtain $\left(\sigma_{2}\right)$ (which is meaningful in the case that the number of cusps is larger than 2) we make a similar construction, but taking into account that parts of $(\Gamma)$ have already been "deleted" with a suitable weight in the construction of $\sigma_{1}$. As we shall see, we will also modify the set $E$ locally around the two points $p_{1}, p_{j_{1}}$ in such a way that $p_{1}$ and $p_{j_{1}}$ becomes regular points of the new set. We start from $\left(\mathcal{G}_{0}^{1}, \Psi_{0}^{1},\left(p_{j_{2}},+1\right)\right)$, where $p_{j_{2}} \in \operatorname{Sing}_{\partial E} \backslash\left\{p_{1}, p_{j_{1}}\right\}$, and $\mathcal{G}_{0}^{1}, \Psi_{0}^{1}$ are obtained as follows. Let

$$
\widetilde{\Psi_{0}^{1}}(z):=\left\{\begin{array}{ll}
\theta_{\Gamma}(z)-2 \sharp\left\{\sigma_{1}^{-1}(z)\right\} & \text { if } z \in\left(\sigma_{1}\right), \\
\theta_{\Gamma}(z) & \text { if } z \in(\Gamma) \backslash\left(\sigma_{1}\right),
\end{array} \quad \widetilde{\mathcal{G}_{0}^{1}}:=\left\{z \in(\Gamma): \widetilde{\Psi_{0}^{1}}(z)>0\right\} .\right.
$$

Since $p_{1}, p_{j_{1}}$ are simple cusp points, for $m \in\left\{1, j_{1}\right\}$ we have

$$
R\left(p_{m}\right) \cap \partial E=R^{+}\left(p_{m}\right) \cap \partial E=\operatorname{graph}\left(\phi_{1}^{m}\right) \cup \operatorname{graph}\left(\phi_{2}^{m}\right),
$$

where $\phi_{1}^{m}, \phi_{2}^{m}$ are functions of class $H^{2, p}$. Now, for $m \in\left\{1, j_{1}\right\}$, we replace $\operatorname{graph}\left(\phi_{1}^{m}\right) \cup \operatorname{graph}\left(\phi_{2}^{m}\right)$ with the support of a curve $\alpha_{m} \in H^{2, p}(0,1)$ such that: $\left(\alpha_{m}\right) \subset R\left(p_{m}\right) ;\left(\alpha_{m}\right) \cap(\Gamma) \cap \operatorname{int}\left(R\left(p_{m}\right)\right)=\left\{p_{m}\right\} ; \alpha_{m}$ joins the two points graph $\left(\phi_{1}^{m}\right) \cap \partial R^{+}\left(p_{m}\right), \operatorname{graph}\left(\phi_{2}^{m}\right) \cap \partial R^{+}\left(p_{m}\right) ; \alpha_{m}$ intersects $(\Gamma)$ tangentially. Then we define

$$
\Psi_{0}^{1}:=\left\{\begin{array}{ll}
\widetilde{\Psi_{0}^{1}} & \text { on } \widetilde{\mathcal{G}_{0}^{1}} \backslash \bigcup_{m \in\left\{1, j_{1}\right\}, l \in\{1,2\}} \operatorname{graph}\left(\phi_{l}^{m}\right), \\
\widetilde{\Psi_{0}^{1}}-1 & \text { on } \bigcup_{m \in\left\{1, j_{1}\right\}, l \in\{1,2\}} \operatorname{graph}\left(\phi_{l}^{m}\right), \\
1 & \text { on }\left(\alpha_{1}\right) \cup\left(\alpha_{j_{1}}\right),
\end{array} \quad \mathcal{G}_{0}^{1}:=\left\{z \in \widetilde{\mathcal{G}_{0}^{1}}: \Psi_{0}^{1}(z)>0\right\} .\right.
$$

By construction $\Psi_{0}^{1}$ verifies the train tracks property on $\mathcal{G}_{0}^{1}$ and every $z \in \mathcal{G}_{0}^{1}$ admits a nice rectangle for $\mathcal{G}_{0}^{1}$ at $z$. Hence we can repeat the construction we used to obtain $\sigma_{1}$ to get $\sigma_{2} \in H^{2, p}(0,1)$ joining $p_{j_{2}}, p_{j_{3}} \in$ $\operatorname{Sing}_{\partial E} \backslash\left\{p_{1}, p_{j_{1}}\right\}$ with $p_{j_{2}} \neq p_{j_{3}}$. Note that $\left(\sigma_{2}\right) \subset(\Gamma)$.

Iterating this argument exactly $M$ times, we obtain the desired $\sigma_{\Gamma} \in \Sigma(E)$. Now we observe that, since $\left(\sigma_{i}\right) \subset(\Gamma), \theta_{\Gamma}(z) \geqslant 2 \sum_{i=1}^{M} \sharp\left\{\sigma_{i}^{-1}(z)\right\}$ by construction and $(\Gamma) \supseteq \partial E$, we also have (57). This concludes the proof of step 1 .

Step 2. Given $\sigma=\left\{\sigma_{1}, \ldots, \sigma_{M}\right\} \in \Sigma(E)$ we can find $\Gamma_{\sigma}=\left\{\gamma_{1}, \ldots, \gamma_{m}\right\} \in \mathcal{A}^{o}(E)$ such that

$$
\mathcal{F}\left(\Gamma_{\sigma}\right)=\int_{\operatorname{Reg}_{\partial E}}\left[1+\left|\kappa_{\partial E}\right|^{p}\right] d \mathcal{H}^{1}+2 \mathcal{F}(\sigma) .
$$

We start noticing that if we set

$$
\mathcal{G}:=\bigcup_{i=1}^{M}\left(\sigma_{i}\right) \cup \partial E, \quad \Psi: \mathcal{G} \rightarrow \mathbb{N} \backslash\{0\}, \quad \Psi(z):= \begin{cases}2 \sum_{i=1}^{M} \sharp\left\{\sigma_{i}^{-1}(z)\right\} & \text { if } z \in \bigcup_{i=1}^{M}\left(\sigma_{i}\right) \backslash \operatorname{Reg}_{\partial E}, \\ 1+2 \sum_{i=1}^{M} \sharp\left\{\sigma_{i}^{-1}(z)\right\} & \text { if } z \in \operatorname{Reg}_{\partial E},\end{cases}
$$

we have that $\mathcal{G}$ admits a nice rectangle at any $z \in \mathcal{G}, \Psi$ verifies the train tracks property on $\mathcal{G}$ and $\partial E=$ $\overline{\{z \in \mathcal{G}: \Psi(z) \equiv 1(\bmod 2)\}}$. Recall that since $\operatorname{Sing}_{\partial E}$ is finite, then $\operatorname{Reg}_{\partial E}$ consists of a finite number of (relative) connected components, whose (relative) boundary is composed by at most two distinct points of Sing ${ }_{\partial E}$.

For every $p_{i} \in \operatorname{Sing}_{\partial E}$ let $R\left(p_{i}\right)$ be a nice rectangle for $\mathcal{G}$ at $p_{i}$ such that $R\left(p_{i}\right) \cap \operatorname{Sing}_{\partial E}=\left\{p_{i}\right\}$. Recall that for every $p_{i} \in \operatorname{Sing}_{\partial E}$ the unit vector $v\left(p_{i}\right)$ normal to $\mathcal{G}$ at $p_{i}$ is such that $R^{+}\left(p_{i}\right) \cap \operatorname{Reg}_{\partial E} \neq \varnothing$ and $R^{-}\left(p_{i}\right) \cap \operatorname{Reg}_{\partial E}=\emptyset$. 
Construction of $\gamma_{1}$. We will construct $\gamma_{1}$ by gluing together the parametrizations of the elements of a finite ordered chain composed by oriented relative connected components of $\operatorname{Reg}_{\partial E}$ and oriented supports of elements of $\sigma$. Let $(\alpha) \subset \mathcal{G}$ be the support of a curve $\alpha$ of class $H^{2, p}$ connecting $p_{i}, p_{j} \in \operatorname{Sing}_{\partial E}$ (with $p_{i}, p_{j}$ not necessarily distinct) such that $\alpha$ has constant speed. From now on by writing $\left(\alpha,\left(p_{i}, p_{j}\right)\right)$ we mean that we move along $(\alpha)$ starting from $p_{i}$ and reaching $p_{j}$.

Let $K_{1}$ be a relative connected component of $\operatorname{Reg}_{\partial E}$. If $K_{1}$ does not have relative boundary then $K_{1}$ is an embedded closed curve of class $H^{2, p}$ and hence we can find a curve $\gamma_{1} \in H^{2, p}\left(S^{1}\right)$ which is a constant speed oriented parametrization of $K_{1}$ and we stop. Let us suppose that $\partial K_{1}=\left\{p_{1}, p_{2}\right\}$ with $p_{1}, p_{2} \in \operatorname{Sing}_{\partial E}$ not necessarily distinct. We set $\mathfrak{F}_{1}=\left(\alpha_{1},\left(p_{1}, p_{2}\right)\right)$, where $\alpha_{1}$ is the arc length parametrization of $K_{1}$. Suppose that we have already constructed a chain

$$
\mathfrak{F}_{i}=\left(\alpha_{1},\left(p_{1}, p_{2}\right)\right),\left(\alpha_{2},\left(p_{2}, p_{3}\right)\right), \ldots,\left(\alpha_{i-1},\left(p_{i-1}, p_{i}\right)\right),\left(\alpha_{i},\left(p_{i}, p_{i+1}\right)\right),
$$

such that

(a) $p_{l} \in \operatorname{Sing}_{\partial E}$ for every $l \in\{1, \ldots, i+1\}$;

(b) if $l$ is odd $\alpha_{l}$ is the arc length parametrization of one of the relative connected components of $\operatorname{Reg}_{\partial E}$ having $p_{l}, p_{l+1}$ as boundary points;

(c) if $l$ is even $\alpha_{l}$ is the arc length parametrization of the support of the unique $\sigma_{i_{l}}$ connecting $p_{l}, p_{l+1}$;

(d) if $l \neq m$ are both odd then $\left(\alpha_{l}\right) \cap\left(\alpha_{m}\right)=\emptyset$; if $l$ is even there is at most an even $m \neq l$ such that $\left(\alpha_{l}\right)=\left(\alpha_{m}\right)$.

Firstly we notice that, since if $l$ is odd (respectively even) $\alpha_{l}$ starts crossing $R^{+}\left(p_{l}\right)$ (respectively $R^{-}\left(p_{l}\right)$ ) and reaches $p_{l+1}$ crossing $R^{+}\left(p_{l+1}\right)$ (respectively $R^{-}\left(p_{l+1}\right)$ ), thanks to Lemma 4.1, we can glue together all the $\alpha_{l}$ in the order, and obtain a unique constant speed curve $\beta_{i} \in H^{2, p}(0,1)$ whose support is given by $\bigcup_{l=1}^{i}\left(\alpha_{l}\right)$. In addition, thanks to (d) we have that $\beta_{i}$ satisfies

$$
\sharp\left\{\beta_{i}^{-1}(z)\right\} \leqslant \Psi(z) \quad \forall z \in\left(\beta_{i}\right),
$$

hence $\beta_{i}$ covers $\left(\sigma_{i}\right)$ at most twice and once each (relative) connected component of $\operatorname{Reg}_{\partial E} \cap\left(\beta_{i}\right)$.

Now if $i$ is odd (respectively even) and we can find a curve $\sigma_{j}$ having as starting or ending point $p_{i+1}$ (respectively a connected component $K$ of $\operatorname{Reg}_{\partial E}$ having $p_{i+1}$ as boundary point) such that there is at most one even $l \in\{1, \ldots, i\}$ such that $\left(\alpha_{l}\right)=\left(\sigma_{j}\right)$ (respectively for every odd $l \in\{1, \ldots, i\}$ we have $\left(\alpha_{l}\right) \neq K$ ), then we set

$$
\mathfrak{F}_{i+1}:=\mathfrak{F}_{i},\left(\alpha_{i+1},\left(p_{i+1}, p_{i+2}\right)\right),
$$

where $\alpha_{i+1}$ is the arc length parametrization of $\left(\sigma_{j}\right)$ (respectively of $K$ ) and $p_{i+2}$ is the other extreme of $\sigma_{j}$ (respectively $\left\{p_{i+1}, p_{i+2}\right\}=\partial K$ ). Otherwise we stop.

Since $\operatorname{Reg}_{\partial E}$ consists of a finite number of (relative) connected components and $\sigma$ consists of a finite number of curves, the above construction stops after a finite number $n$ of steps. It is immediate to check that $n>3$. We claim that $n$ is even and $p_{n+1}=p_{1}$. Suppose by contradiction that $n$ is odd. Then $\alpha_{n}$ parametrizes a connected component $\widetilde{K}$ of $\operatorname{Reg}_{\partial E}$ such that $\left\{p_{n}, p_{n+1}\right\}$ is the relative boundary of $\widetilde{K}$ and $n \geqslant 5$. Furthermore as our construction stops, there are two even numbers $l, m \in\{1, \ldots, n-1\}, l<m$ such that $\left(\alpha_{l}\right)=\left(\alpha_{m}\right)=\left(\sigma_{j}\right)$, where $\sigma_{j}$ is the unique element of $\sigma$ having $p_{n+1}$ as starting or ending point.

This means that we crossed twice $R^{-}\left(p_{n+1}\right)$ at the step $m \leqslant n-1$. If $l \leqslant m<n-1$, since there is at most only another relative connected component of $\operatorname{Reg}_{\partial E}$ having $p_{n+1}$ as a boundary point, in view of (d) we have a contradiction. If $m=n-1$ then $\left(\alpha_{n-1}, p_{n+1}, p_{n}\right)$ and $l \leqslant n-3$ (notice that $n-3 \geqslant 2$ as $n \geqslant 5$ ). Therefore as in the previous case since there is at most only another relative connected component of $\operatorname{Reg}_{\partial E}$ having $p_{n+1}$ as a boundary point, in view of (d) we have a contradiction. Hence $n$ is even. With a similar argument and using the fact that $n$ is even, one can prove that $p_{n+1}=p_{1}$.

As already noticed, we can find a constant speed curve $\beta_{n} \in H^{2, p}(0,1)$ such that $\left(\beta_{n}\right)=\bigcup_{l=1}^{n}\left(\alpha_{l}\right)$. In addition, as $p_{n+1}=p_{1}$ and $\alpha_{n}$ reaches $p_{1}$ crossing $R^{-}\left(p_{1}\right)$, while $\alpha_{1}$ moves from $p_{1} \operatorname{crossing} R^{+}\left(p_{1}\right)$, we can find a 
constant speed curve $\gamma_{1} \in H^{2, p}\left(S^{1}\right)$ such that $\left(\gamma_{1}\right)=\left(\beta_{n}\right)$ and also by $(61) \sharp\left\{\gamma_{1}^{-1}(z)\right\}=\sharp\left\{\beta_{n}^{-1}(z)\right\} \leqslant \Psi(z)$ for every $z \in\left(\gamma_{1}\right)$.

Construction of $\gamma_{2}$. To obtain $\gamma_{2}$ we repeat the same construction used to obtain $\gamma_{1}$, but this time we start from $\mathcal{G}_{1}:=\left\{z \in \mathcal{G}: \Psi_{1}(z)>0\right\}$, where $\Psi_{1}(z):=\Psi(z)-\sharp\left\{\gamma_{1}^{-1}(z)\right\}$ and taking into account that $\gamma_{2}$ verifies $\sharp\left\{\gamma_{2}^{-1}(z)\right\} \leqslant \Psi_{1}(z)$.

Since the number of connected components of $\operatorname{Reg}_{\partial E}$ is finite and each component of $\sigma$ starts and ends at a boundary point of a relative connected component of $\operatorname{Reg}_{\partial E}$, iterating this procedure we obtain $\Gamma_{\sigma}:=$ $\left\{\gamma_{1}, \ldots, \gamma_{m}\right\}$ such that $\left(\Gamma_{\sigma}\right)=\bigcup_{i=1}^{M}\left(\sigma_{i}\right) \cup \partial E$ and $\theta_{\Gamma_{\sigma}}=\Psi$. Hence (60) holds. Moreover, since by construction $\overline{\left\{z \in\left(\Gamma_{\sigma}\right): \theta_{\Gamma_{\sigma}}(z) \equiv 1(\bmod 2)\right\}}=\partial E^{*}$, by Proposition 3.13 we also have $\Gamma_{\sigma} \in \mathcal{A}^{o}(E)$.

As a consequence of step 2 and (42) we get

$$
\overline{\mathcal{F}}(E) \leqslant \int_{\operatorname{Reg}_{\partial E}}\left[1+\left|\kappa_{\partial E}\right|^{p}\right] d \mathcal{H}^{1}+2 \inf _{\sigma \in \Sigma(E)} \mathcal{F}(\sigma) .
$$

Hence by step 1 we deduce

$$
\overline{\mathcal{F}}(E)=\int_{\operatorname{Reg}_{\partial E}}\left[1+\left|\kappa_{\partial E}\right|^{p}\right] d \mathcal{H}^{1}+2 \inf _{\sigma \in \Sigma(E)} \mathcal{F}(\sigma) .
$$

The proof of the theorem then follows from the following final step.

Step 3. There exists $\bar{\sigma} \in \Sigma(E)$ such that

$$
\int_{\operatorname{Reg}_{\partial E}}\left[1+\left|\kappa_{\partial E}\right|^{p}\right] d \mathcal{H}^{1}+2 \mathcal{F}(\bar{\sigma})=\int_{\operatorname{Reg}_{\partial E}}\left[1+\left|\kappa_{\partial E}\right|^{p}\right] d \mathcal{H}^{1}+2 \inf _{\sigma \in \Sigma(E)} \mathcal{F}(\sigma) .
$$

Let $\Gamma$ be a minimal element in $\mathcal{A}(E)$. Thanks to Theorem 6.3 we can pick a sequence $\left\{\Gamma_{n}\right\} \subset H^{2, p}(\mathbb{S}) \cap \mathcal{Q}_{\text {fin }}(E)$ converging to $\Gamma$ in $H^{2, p}$. Let $\sigma_{\Gamma_{n}}$ be the elements of $\Sigma(E)$ constructed in step 1, i.e., such that (57) holds with $\Gamma$ replaced by $\Gamma_{n}$. Since $\left(\sigma_{\Gamma_{n}}\right) \subset\left(\Gamma_{n}\right)$ we get

$$
\overline{\mathcal{F}}(E) \leqslant \limsup _{n \rightarrow \infty} \int_{\operatorname{Reg}_{\partial E}}\left[1+\left|\kappa_{\partial E}\right|^{p}\right] d \mathcal{H}^{1}+2 \mathcal{F}\left(\sigma_{\Gamma_{n}}\right) \leqslant \lim _{n \rightarrow \infty} \mathcal{F}\left(\Gamma_{n}\right)=\overline{\mathcal{F}}(E) .
$$

Due to the strong convergence of the sequence $\left\{\Gamma_{n}\right\}$ and the finiteness of $\operatorname{Sing}_{\partial E}$, we can find a subsequence of $\left\{\sigma_{n}\right\}$ which converges to a certain $\bar{\sigma} \in \Sigma(E)$. Using the lower semicontinuity of $\mathcal{F}$ on $\Sigma(E)$ we have (55).

Eventually let us sketch very briefly how the representation formula (55) can be proved removing the hypothesis that every element of $\operatorname{Sing}_{\partial E}$ is a simple cusp point (see [5] for a more detailed proof). Let $M:=$ $\frac{1}{2} \sum_{p \in \operatorname{Sing}_{\partial E}} \omega_{\partial E}(p)$. Recall that $M \in \mathbb{N}$ by Theorem 8.4. In order to consider each singular point with the correct multiplicity, let us represent the set $\left\{q \in \operatorname{Sing}_{\partial E}: \omega_{\partial E}(q) \neq 0\right\}=\left\{\widehat{p}_{1}, \ldots, \widehat{p}_{d}\right\}$ as follows:

$$
\left\{q \in \operatorname{Sing}_{\partial E}: \omega_{\partial E}(q) \neq 0\right\}=\left\{p_{1}, p_{2}, \ldots, p_{2 M}\right\},
$$

where $d \leqslant 2 M, p_{j}:=\widehat{p}_{1}$ for every $1 \leqslant j \leqslant \omega_{\partial E}\left(p_{1}\right)$, and $p_{j}:=\widehat{p}_{i}$ for every $j$ with $\sum_{h=1}^{i-1} \omega_{\partial E}\left(\widehat{p}_{h}\right) \leqslant j \leqslant$ $\sum_{h=1}^{i} \omega_{\partial E}\left(\widehat{p}_{h}\right)$ and every $i=2, \ldots, d$.

Definition 8.7. We define $\Sigma(E)$ as the set of all collections $\left\{\sigma_{1}, \ldots, \sigma_{M}\right\}$ of curves such that properties (i) and (ii) of Definition 8.5 hold, and

(iii) $\sigma_{i}(0), \sigma_{i}(1) \in \operatorname{Sing}_{\partial E}$ for every $i \in\{1, \ldots, M\}$, and there exists a bijective application between $\left\{\sigma_{1}(0)\right.$, $\left.\sigma_{1}(1), \ldots, \sigma_{M}(0), \sigma_{M}(1)\right\}$ and $\left\{q \in \operatorname{Sing}_{\partial E}: \omega_{\partial E}(q) \neq 0\right\}$; 


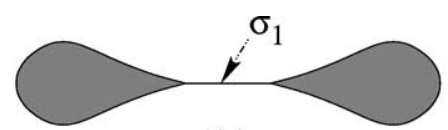

(a)

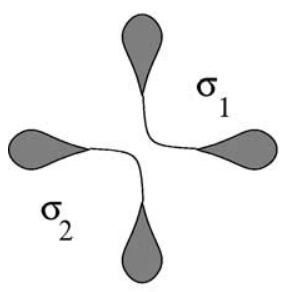

(b)

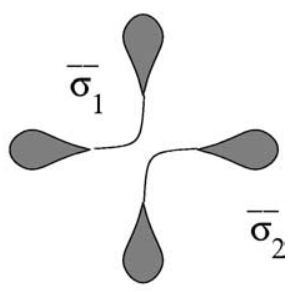

(c)

Fig. 10. (a) shows the easiest example of formula (55): if $E$ is the set consisting of two drops (as in Fig. 1), then $\overline{\mathcal{F}}(E)$ equals $\int_{\operatorname{Reg}_{\partial E}}\left[1+\left|\kappa_{\partial E}\right|^{p}\right] d \mathcal{H}^{1}$ plus twice the distance between the two simple cusp points. (b) and (c) (where $E$ consists of four drops) show that $\Sigma(E)$ does not necessarily reduce to a unique possible collection $\left\{\sigma_{1}, \ldots, \sigma_{M}\right\}$.

(iv) for every $i \in\{1, \ldots, M\}$ either

$$
\frac{d \sigma_{i}}{d t}(0) \text { is parallel to } R_{c} v\left(\sigma_{i}(0)\right) \text { and } \frac{d \sigma_{i}}{d t}(1) \text { is parallel to } R v\left(\sigma_{i}(1)\right)
$$

or

$$
\frac{d \sigma_{i}}{d t}(0) \text { is parallel to } R v\left(\sigma_{i}(0)\right) \text { and } \frac{d \sigma_{i}}{d t}(1) \text { is parallel to } R_{c} v\left(\sigma_{i}(1)\right) \text {, }
$$

where $R_{c}$ (respectively $R$ ) denotes the rotation of $\pi / 2$ in clockwise (respectively counterclockwise) order.

Notice that (iii) implies that for $i \in\{1, \ldots, m\}$ we have $\sigma_{i}(0)=p_{i_{0}}, \sigma_{i}(1)=p_{i_{1}} \in \operatorname{Sing}_{\partial E}$ and $i_{0} \neq i_{1}$ (however the points $p_{i_{0}}$ and $p_{i_{1}}$ may coincide).

The proof of (55) then follows by

(a) suitably approximating $E$ with a sequence $\left\{E_{n}\right\}$ of sets obtained by modifying $E$ locally around each point of $\operatorname{Sing}_{\partial E}$ in such a way that $\partial E_{n}=\partial E$ outside the union of a family of nice rectangles of diameter strictly smaller than $1 / 2^{n}$ covering $\operatorname{Sing}_{\partial E}$, and in addition $\operatorname{Sing}_{\partial E_{n}}$ is composed only by simple cusp points;

(b) suitably passing to the limit as $n \rightarrow+\infty$ in the formula (55) where $E$ is replaced by $E_{n}$, which is valid thanks to Theorem 8.6, see [5].

\subsection{A counterexample}

Using Theorem 8.6 we show an example of a set $E$ for which the minimum in (44) is not attained.

Proposition 8.8. There exists a set $E$ with $\sharp \operatorname{Sing}_{\partial E}=2$ such that $\overline{\mathcal{F}}(E)<+\infty$ and the minimum of $\mathcal{F}$ over the class $\mathcal{Q}_{\text {fin }}(E)$ in (44) is not achieved.

Proof. Let $E_{j}$ with $j=1,2,3$ be as in Fig. 7: they are three connected sets, whose closure are pairwise disjoint. The set $E_{3}$ is smooth and contained in $\{y \geqslant 0\}$, while $E_{i}, i=1,2$, are smooth except for the point $p_{i}$, which is a simple cusp point, $p_{1}=(-1,0), p_{2}=(0,1)$. The unoriented tangent to $\partial E_{i}$ at $p_{i}$ is the $x$-axis, $i=1,2$. We 
suppose that the oscillating part of $\partial E_{3}$ touches the segment $]-1,1[\times\{0\}$ an infinite number of times. Since the segment joining $p_{1}$ and $p_{2}$ is an absolute minimizer for $\mathcal{F}$ in $\Sigma(E)$ the thesis follows from Theorem 8.6.

Corollary 8.9. There exists a set $E \subset \mathbb{R}^{2}$ such that $\overline{\mathcal{F}}(E)<+\infty$, with only two simple cusp points and such that the minimal system in $\mathcal{A}(E)$ has multiplicity equal to 3 on a set of positive $\mathcal{H}^{1}$ measure.

Proof. It is enough to choose, in Proposition 8.8, the set $E_{3}$ in such a way that $\partial E_{3}$ intersects $]-1,1[\times\{0\}$ on a set of positive $\mathcal{H}^{1}$ measure.

\section{References}

[1] L. Ambrosio, N. Fusco, D. Pallara, Functions of Bounded Variation and Free Discontinuity Problems, Oxford Science Publication, 2000.

[2] L. Ambrosio, C. Mantegazza, Curvature and distance function from a manifold, J. Geom. Anal. 5 (1998) 723-748.

[3] L. Ambrosio, S. Masnou, A direct variational approach to a problem arising in image reconstruction, Interfaces and Free Boundaries, submitted for publication.

[4] G. Bellettini, G. Dal Maso, M. Paolini, Semicontinuity and relaxation properties of a curvature depending functional in 2D, Ann. Scuola Norm. Sup. Pisa Cl. Sci. (4) 20 (1993) 247-299.

[5] G. Bellettini, L. Mugnai, Characterization and representation of the lower semicontinuous envelope of the elastica functional, Preprint Univ. Pisa, May 2003.

[6] G. Bellettini, M. Paolini, Variational properties of an image segmentation functional depending on contours curvature, Adv. Math. Sci. Appl. 5 (1995) 681-715.

[7] H. Cartan, Theorie Elementaire des Fonctions Analytiques d'Une ou Pluiseurs Variables Complexes, Hermann, 1961.

[8] A. Coscia, On curvature sensitive image segmentation, Nonlin. Anal. 39 (2000) 711-730.

[9] B. Dacorogna, Direct Methods in the Calculus of Variations, Springer-Verlag, 1989.

[10] S. Delladio, Special generalized gauss graphs and their application to minimization of functionals involving curvatures, J. Reine Angew. Math. 486 (1997) 17-43.

[11] L. Euler, Additamentum I de curvis elasticis, methodus inveniendi lineas curvas maximi minimive proprietate gaudentes, Opera Omnia I (24) (1744) 231-297.

[12] H. Federer, Geometric Measure Theory, Springer-Verlag, 1969.

[13] M. Giaquinta, S. Hildebrandt, Calculus of Variations I, in: Grundleheren der Mathematischen Wissenschaften, vol. 310, Springer-Verlag, 1996.

[14] E. De Giorgi, Some remarks on $\Gamma$-convergence and least squares method, in: Proc. Composite Media and Homogeneization Theory, Trieste, 1991, pp. 135-142.

[15] O. Gonzales, J.H. Maddocks, F. Schuricht, H. von der Mosel, Global curvature and self-contact of nonlinearly elastic curves and rods, Calc. Var. Partial Differential Equations 14 (2002) 29-68.

[16] J.E. Hutchinson, $\mathcal{C}^{1, \alpha}$-multiple functions regularity and tangent cone behaviour for varifolds with second fundamental form in $L^{p}$, Proc. Symp. Pure Math. 44 (1986) 281-306.

[17] A.E.H. Love, A Treatise on the Mathematical Theory of Elasticity, Dover, 1944.

[18] S. Masnou, Disocclusion: a variational approach using level lines, IEEE Trans. Image Process. 11 (2002) 68-76.

[19] S. Masnou, J.M. Morel, Level lines based disocclusion, in: Proc. ICIP’98 IEEE Internat. Conf. on Image Processing, 1998, pp. $259-263$.

[20] J.M. Morel, S. Solimini, Variational Methods in Image Segmentation, in: Progress in Nonlinear Differential Equations and Their Applications, vol. 14, Birkhäuser, 1995.

[21] D. Mumford, Elastica and computer vision, in: Algebraic Geometry and its Applications, 1994, pp. 491-506.

[22] D. Mumford, M. Nitzberg, T. Shiota, Filtering, Segmentation and Depth, in: Lecture Notes in Computer Science, vol. 662, Springer-Verlag, 1993.

[23] M. Nitzberg, D. Mumford, The 2.1-D sketch, in: Proc. of the Third Internat. Conf. on Computer Vision, Osaka, 1990.

[24] L. Simon, Existence of surfaces minimizing the Willmore functional, Comm. Anal. Geom. 2 (1993) 281-326.

[25] T.J. Willmore, An introduction to Riemannian Geometry, Clarendon Press, 1993. 